\title{
A Flight Sensory-Motor to Olfactory Histamine Circuit Mediates Olfactory Processing of Ecologically and Behaviorally Natural Stimuli
}

Samual P. Bradley

sbradle7@mix.wvu.edu

Follow this and additional works at: https://researchrepository.wvu.edu/etd

Part of the Systems Neuroscience Commons

\section{Recommended Citation}

Bradley, Samual P., "A Flight Sensory-Motor to Olfactory Histamine Circuit Mediates Olfactory Processing of Ecologically and Behaviorally Natural Stimuli" (2020). Graduate Theses, Dissertations, and Problem Reports. 7634.

https://researchrepository.wvu.edu/etd/7634

This Dissertation is protected by copyright and/or related rights. It has been brought to you by the The Research Repository @ WVU with permission from the rights-holder(s). You are free to use this Dissertation in any way that is permitted by the copyright and related rights legislation that applies to your use. For other uses you must obtain permission from the rights-holder(s) directly, unless additional rights are indicated by a Creative Commons license in the record and/ or on the work itself. This Dissertation has been accepted for inclusion in WVU Graduate Theses, Dissertations, and Problem Reports collection by an authorized administrator of The Research Repository @ WVU.

For more information, please contact researchrepository@mail.wvu.edu. 
A Flight Sensory-Motor to Olfactory Histamine Circuit Mediates Olfactory Processing of Ecologically and Behaviorally Natural Stimuli

\author{
Samual P. Bradley
}

Dissertation submitted to the Eberly College of Arts and Sciences at West Virginia University

In partial fulfillment of the requirements for the degree of

Doctor of Philosophy in Biology

\author{
Kevin C. Daly, Ph.D., Chair \\ Andrew M. Dacks, Ph.D. \\ Sarah M. Farris, Ph.D. \\ Gary Marsat, Ph.D. \\ Sergiy Yakovenko, Ph.D. \\ Department of Biology
}

Morgantown, West Virginia

2019

Keywords: Neurobiology, olfaction, corollary discharge, histamine

Copyright 2019 Samual P. Bradley 


\begin{abstract}
A Flight Sensory-Motor to Olfactory Histamine Circuit Mediates Olfactory Processing of Ecologically and Behaviorally Natural Stimuli
\end{abstract}

\author{
Samual P. Bradley
}

Environmental pressures have conferred species specific behavioral and morphological traits to optimize reproductive success. To optimally interact with their environment, nervous systems have evolved motor-to-sensory circuits that mediate the processing of its own reafference. Moth flight behavioral patterns to odor sources are stereotyped, presumably to optimize the likelihood of interacting with the odor source. In the moth Manduca sexta wing beating causes oscillatory flow of air over the antenna; because of this, odorant-antennal interactions are oscillatory in nature. Electroantennogram recordings on antennae show that the biophysical properties of their spiking activity can effectively track odors presented at the wing beat frequency. Psychophysical experiments using Manduca show that when odors are pulsed, as opposed to presented as a continuous stream, detection and discrimination thresholds are lowered. In this study, we characterized histamine immunoreactivity in the thoracic ganglia and brain of Manduca. We generated antibodies for and characterized the distribution of the histamine B receptor, the first known antibody for this receptor protein. Our results show an elaborate pair of neurons projecting from the mesothoracic ganglion to the brain, including axon innervation of the antennal lobe and antennal mechanosensory and motor centers. Additionally, histamine B receptor labeling overlapped with a subset of GABAergic and peptidergic local interneurons. Next, we characterized the response properties of these cells within the context of fictive flight behavior and found a tonic increase in activity. Furthermore, disrupting this circuit, with surgical ablation and pharmacology, disrupts antennal lobe projection neurons from entraining to odors presented at a natural $20 \mathrm{~Hz}$ frequency, as well as behavioral measures of detection and discrimination thresholds. Finally, we characterized the relationship between motor patterns/behaviors, and circuit structure of this pair of histamine immunoreactive neurons. Specifically, presence of MDHn axon collaterals entering the antennal lobe is correlated with olfactory-guided target approach behaviors in crepuscular and nocturnal moths who require stereotyped zigzagging and wing beating behaviors for locating an olfactory target have axonal ramifications in the antennal lobe. This study is the first characterization of a motor to olfactory corollary discharge circuit in invertebrates and may represent the first characterization of a higher order corollary discharge circuit in an invertebrate model. 


\section{Table of Contents}

Literature Review

Chapter 1

7

Chapter 2

Chapter 3

Discussion

References

Acknowledgements

Appendix A

50

Appendix B

78

Appendix C 


\section{Literature Review}

The forces driving evolution have afforded various strategies to optimize fitness. For example, plants evolved the ability to convert, with the help of energy from the sun, carbon dioxide and water into sugars they use for the production of cellular energy; or single celled organisms evolved cilia to facilitate movements through their environment to find food. Alternatively, animals evolved nervous systems that govern more complex behaviors that facilitate survival and reproduction. These complex networks of cells evolved with cnidarians just prior to bilaterala species of more complex animals, with sponges and placozoans being the only species of animal without one (Holland, 2003). Nervous systems provided a basis for selection that increased fitness by allowing the sensing of stimuli coupled with complex coordinated motor behaviors. This strategy has shown a high level of persistence over species and generations, but the details of how nervous systems function remains one of the most sought-after questions in science. Here we take an evolutionary and neuroethological approach towards neural computation where aspects of the animal's environmental niche and their behavioral state mediate the processing of sensory stimuli.

\section{Affordances and active sensing}

Agents navigating the environment are directly beholden to that environment, as well as the sensory receptors that determine the type of information that can enter the nervous system. Though animals are rarely stagnant; they often are mobile while they sense their environment. Even stationary predators like frogs require visual tracking with their eyes which govern their ability to catch flies. So, it's not just the environmental features available as determined by the world's features and the agent's sensory receptors, but the complicated working relationship between the environment and the agent. For example, consider the case of a moth and a flower. Odor plumes emanating from a flower are random in nature; volatiles move through the air with the help of the wind whose strength and direction is relatively unpredictable from moment to 
moment. Likewise, the flight patterns of a fruit fly evading the pursuit of a dragonfly is also chaotic and difficult to predict from moment to moment. And yet the flying moth and dragonfly get their reward at a high rate of success. Both cases, as we will see, require stereotypical behaviors that increase the reliability of successful goal-driven behaviors in a chaotic environment. In 1979, JJ Gibson (1979) expounded his theory of nervous system functioning by changing the discussion toward the interaction of the agent and the environment. In doing so, he used the term affordance. In his view, the environment "offers" something to the animal, and how the animal and the environment is "complementarity".

An example demonstrating the concept of affordances is dynamic touch or haptic perception. This is the process of extracting information from grasping and manipulating objects independent of visual input. Here the contraction and distortion of muscles and tendons needed to support the object, the pressure changes of your fingertips, and the force on your wrist and arm as they fight the effect of gravity on the object (Gibson, 1962; Chemero, 2011). Perhaps more impressive is the ability of humans to determine the length of a rod-like object based solely on one form of haptic perception. In these studies, experimenters shielded individuals from the bar and were instructed to grasp the bar in the middle. The only manipulations the subjects could perform was to rotate the bar with respect to their wrist. Subjects were then instructed to turn a wheel which shortened or lengthened a piece of string as an estimate of its length. Subjects were remarkably accurate at determining the length of the rod. The authors reasoned that the rotational inertia of the object when manipulated provided sufficient information for length determination (Fitzpatrick et al., 1994).

Haptic perception is also an example of active sensing where the perceptual processes are modulated by the animal's behavior (Wachowiak, 2011). Though others have described all movement or action effectively active sensing (Feldman, 2016). This phenomenon appears 
throughout animals and across sensory domains and is another relation between the animal and the environment. One interesting example is the act of sniffing in mammalian olfaction. Mammals use inhalation through the nasal cavities for respiration which happens to be the same necessary mechanism that governs the flow of odorant molecules to the nasal cavity so that they can bind to olfactory receptor neurons in the olfactory epithelium. Important here is that motor system action is necessarily linked to the ability to process sensory information. Behavioral studies show that mice produce stereotypical sniff behaviors in response to novel odor stimuli. Additionally, sniff frequency is dependent on the behavioral demands of the animal. For example, the details of goal directed behavior shows variable effects on changes in sniff frequency (Wesson et al., 2008) with different response patterns of the major outputs of the olfactory bulb, the mitral and tufted cells, in response to sniff frequency changes (Verhagen et al., 2007). Additionally, while the details are lacking, there exist serotonergic projections from the Raphe nucleus which also modulates respiration and hence inhalation motor patterns (McClean and Shipley, 1987).

\section{Dragonfly prey capture}

One recent example examining the role of movement and sensing information is the invertebrate dragonflies. Dragonflies are some of the most ancient insect species. They are vivacious predators of other insects and show a remarkable ability to track and catch their prey. These highly visual insects show quick reflexes darting towards a chaotic moving fly; gathering their food in their hairy arms before indulging in their catch. There are various mechanisms governing this ability, though it does appear to use an interception technique. In this particular interception technique, the dragonfly works to maintain the placement of the prey on the retina. These insects have a specialized set of 8 pairs of neurons that project from the visual system to the flight and leg motor neurons in the prothoracic, mesothoracic and metathoracic ganglia. These cells are driven by small object motion sensitive cells in the retina that respond to objects approximately the size of their prey and as they move across their visual field. This direct connection and given 
their large diameter axons represent a fast yet reasonable explanation for their behavior (Olberg et al., 2000; Gonzalez-Bellido et al., 2013). These hypotheses suggest that visual sensory information drives reactionary motor movements that maintain stable prey foveation. By comparison, consider a tracking behavior called continuous pursuit in Land and Collett (1974), where they found houseflies participating in chasing behavior is modeled by action reaction type mechanisms. Here the visual system detects the flight course of the leading fly, and the tracking fly alters its path to mimic the twists and turns of the leading fly (Olberg et al., 2000).

However, other research in this area suggests that these mechanisms are not consistent with the flight behavior of dragonfly prey capture. As Mischiati and colleagues (2015) suggest, the speed and complexity of maneuvers for successful prey capture is beyond what sensory feedback can provide. In their study, they measured head and body movements in response to animal "capture" of computer-generated prey. Their study suggested that the timing of head and body movements are inconsistent with parallel navigation. This is most notably seen in the uncorrelated nature of prey movement and dragonfly movements. These studies found that instead the dragonfly exhibits a stereotyped body alignment directly under the prey which it then homes in upon. These body movements would cause apparent drift of prey on its retina. However, the dragonfly rotates its head in the opposite direction to cancel this apparent motion. Furthermore, it does so by keeping the prey directly over the fovea on its head as it rotates; this occurs instantaneously with body movements suggesting that compensation is not from sensory feedback but rather from an internal model of body movement. While the neural circuitry responsible for transmitting this information is unknown, this represents an example of how the nervous system functions to synchronize its behavior with its environment to optimize behavioral goals. 


\section{What you will find in here}

In the following study, we investigate a circuitry that connects flight sensory motor neurons to the olfactory system. In chapter 1 we show a detailed characterization of a pair of histamineimmunoreactive neurons. These cells have their cell bodies in the dorsal mesothoracic ganglia and ascend to the deutocerebrum with a small subset of axon collaterals that penetrate the antennal lobe. An antibody made against the Manduca histamine B receptor shows a widespread distribution of a small number of local interneurons who modulate antennal lobe activity. These ascending histamine immunoreactive cells are present in the larvae of Manduca although their detailed structure differs between larva and adult as well as there being no evidence of histamine $B$ receptor labeling within the antennal lobe in particular, suggesting these cells play different functional roles at different stages of the animals life cycle.

We propose that this circuit mediates the ability of this species to process high frequency natural stimuli possibly set up by wing beating behavior. Specifically, we hypothesize that 1) disrupting this circuit will decrease the ability of antennal lobe neurons to track natural stimuli, and 2) a functional circuit from the thoracic ganglia to the olfactory system is complete in night flying Lepidoptera and not in day dwelling Lepidoptera and other insect species. We do this by taking two different approaches. First, we compare the mesothoracic deutocerebrum histamine circuit structure across insect species. Most importantly, we show that a functional mesothoracic ganglion to antennal lobe circuit only exists in night flying plume tracking insects such as moths, but not day flying insects like butterflies. At first glance, this relationship seems counter to what one would expect from phylogenetic relationships with the suborder macrolepidoptera having both moths and butterflies and microlepidoptera also has moths and butterflies. However, flight behavior of these does correlate with functional circuitry. Moths who show wingbeat effects on antennal airflow or use wing beating (even without an apparent reason) to locate an odor source, possess a complete circuit to the antennal lobe. 
Second, we use electrophysiology and neuropharmacology to determine the effects this circuit has on antennal lobe odor processing and behavior. In collaboration with Phillip Chapman we 1) first characterized the response of the mesothoracic to deutocerebrum histamine neurons to induced fictive flight using the octopamine agonist chlordimeoform. Chapman found that activation of the flight neural circuitry induced a tonic response from MDH neurons. 2) in a series of experiments, I and Benjamin Houot characterized the response of antennal lobe neurons to temporally structured odor stimulation before and after ablation of the ventral nerve cord, and before and after histamine and histamine receptor antagonist application to determine the consequence of histamine release on the antennal lobe frequency response. In these experiments both nerve cord ablation and histamine blockade in the brain disrupted antennal lobe neurons ability to track odors pulsed at wingbeat frequencies. 3) Finally, we evaluated the consequence of histamine function on behavioral measures of odor detection and discrimination thresholds. Here we found that histamine generally enhances olfactory acuity. Together these results suggest that the MDH circuitry optimizes odor processing within the context of odor-guided flight. 


\section{Chapter 1}

The information here is a summary of the full research article published in Bradley et al. 2015 in Frontiers in Neural Circuits; for more information on the methodology and the results of the study, see appendix A. In this chapter my personal contributions were the immunohistamine labeling in adult Manduca, severing the ventral nerve cord to verify antennal lobe histamine immunoreactivity

originated in the thoracic ganglia, and the dual histamine immunolabeling with GABA, FMRFamide and allatotropin. 


\section{Introduction}

\section{Manduca sexta}

The primary animal of interest in the following studies is the moth Manduca sexta. Manduca is a large ( $>5 \mathrm{~cm}$ wingspan) brown moth with yellow spots along its abdomen with large green larvae possessing a characteristic horn at its posterior end. Phylogenetically, Manduca is of the class insecta, the order lepidoptera and the family Sphingidae, also known as a hawkmoth and sometimes referred to as the Sphinx moth. The superfamily of Sphingidae is Bombycoidea which includes the silk moth Bombyx mori, which are in the family Noctuidae (Dai et al., 2016). Hawkmoths are characterized by a long thin proboscis and a characteristic hovering behavior while feeding on plants, including swaying back and forth to avoid predation (Kitching, 2002). This hovering behavior occurs 3 times in the animal kingdom, representing an interesting example of convergent evolution with hummingbirds and bats (Voigt and Winter, 1999). Hawkmoths and other sphingids show a co-evolution with Datura wrightii who's flowers are rich in sucrose at the pit of long narrow tubular corollas. The flowers of this species are light in color opening at dusk and dawn consistent with the hawkmoths being crepuscular (Kitching, 2002).

Manduca has been used as a model organism in developmental biology, neurobiology, immunology and flight biomechanics. Here, we are interested in the relationship between their olfactory system and flight mechanics, particularly the reafferent effects of wing beating on olfactory processing. The olfactory system of Manduca was anatomically and physiologically characterized starting in the 1970s. One finding was the presence of a pair of histamine immunoreactive neurons that project from the mesothoracic ganglia to the antennal lobe (Homberg 1994). Additional studies later showed these moths can learn to respond to odors with a feeding response which subsequently provided the means for psychophysical studies that 
identified specific thresholds for odor detection and discrimination (Daly and Smith, 2000; Daly et al., 2001).

The biomechanics of Manduca flight has been described previously by Willmott and Ellington (1997). Like all plume tracking insects, moths show a "zig-zagging" pattern as they cast in and out of plumes (Willis and Arbas, 1991). This casting behavior is dependent on the intermittency of the plume, demonstrating a tight link between a natural stimulus and the evolution of behavior (Willis and Baker, 1984; Baker et al., 1985). Riding atop the low frequency zig-zagging behavior is high frequency oscillations over their antenna. This disturbance is caused when the downstroke of the wingbeat forces air over the antennae (Sane, 2006) which has the capacity to affect odor penetration into the antenna's sensillar array (Loudon and Koehl, 2000). Primary olfactory neurons can track odors that are pulsed at the wing beat frequency and pulsing decreases behavioral detection thresholds (Tripathy et al. 2010; Daly et al., 2013; Houot, et al., 2014). Together these findings suggest that Manduca evolved to process complex olfactory stimuli that could be structured optimally for the nervous system, but how wing action and olfaction are aligned is not clear.

\section{Thoracic Ganglia}

One possibility is for the animal's flight motor systems to communicate with the olfactory system to modulate neural responses in real time. Ancestrally, the thoracic ganglia were composed of 3 separate ganglia (prothoracic, mesothoracic and metathoracic ganglia anterior to posterior) with 11 abdominal ganglia posterior to the thoracic ganglion (Niven et al., 2008). In Manduca, along with most other insects, the mesothoracic, metathoracic and the first 2 abdominal ganglia are fused into one structure. The thoracic ganglia contain motor pattern generating circuits along with motor neurons that control wing and leg behaviors, as well as mechanosensory and proprioceptive sensory cells. The mesothoracic ganglion houses the motor neurons for the 
forewing elevator and depressor muscles (Rind, 1983), and hence drives wing beating. The functionality of motor circuits depends on interneurons that control the rhythmic nature to motor activity called central pattern generators. These cells fire continuously and rhythmically through reciprocally inhibiting connections, though the precise functionality of this circuitry is not clearly established.

\section{Olfactory processing in insects}

Olfaction, along with taste, makes up the chemosensory processing systems within the nervous system. Chemicals such as plant volatiles and pheromones bind to sensory receptors housed within the sensilla in the antenna and maxillary palps. Receptor proteins transduce bound molecules into an electrical impulse which is transmitted to primary olfactory processing centers, the antennal lobe $(A L)$ in insects, olfactory lobe in crustaceans and olfactory bulb in mammals. Antennal lobe projections continue along three output tracts to the mushroom bodies and lateral horn in the protocerebrum. Along this pathway, odors are believed to be encoded and transformed into motor movements and subsequent behaviors.

Odor molecules that contact the antenna diffuse through pores in the cuticle into the antennal lymph. Carrier proteins located in the lymph escort bound odorant molecules to receptor proteins. The molecule binds to the receptor and causes a conformational change in the protein, detaches and is inactivated by degrading enzymes. This process occurs in a very short time frame ( $2 \mathrm{~ms})$ and is believed, at least partially, responsible for characterizing odorant receptors as flux detectors (Kaissling, 2001). Odorant receptors differ between insect species both in number and response profile due to evolutionary pressures. The most extensive research on olfactory receptor neurons has been in Drosophila melanogaster. In this species, there are approximately 1200 olfactory receptor neurons which house 61 different olfactory receptor types, each encoded by a distinct gene (Hallam et al., 2004; Vosshall et al., 2000). Olfactory receptors are seven 
transmembrane domain non-selective cation channels (Sato et al., 2008) possessing a metabotropic component as well (Wicher et al., 2008). Approximately two thirds of olfactory receptors also express the coreceptor Or83b (Vosshall et al., 1999) which is required for proper ORN signaling in these cells (Larson et al., 2004). The response profile of olfactory receptor neurons is dependent on the receptors expressed in those neurons. Each receptor shows a particular response tuning curve to odors. Some receptors are broadly tuned whereas others respond to only one known ligand (Stensmyr et al., 2012); additionally, there is receptor specific variability in the duration of the neural response, and whether it is excitatory or inhibitory (Hallem et al., 2004). Individual receptor neurons can express multiple receptors and the response properties of these cells is a summation of both receptors. Finally, different receptors respond variably to changes in concentration; where some fall off sharply with a decrease in log step concentration and others showing remarkable sensitivity (Hallem et al., 2004).

Each olfactory receptor neuron projects to the same olfactory glomerulus (Vosshall et al., 2000). Each olfactory glomerulus is encapsulated in glial cells that spatially and to some degree electrically separate different glomeruli. In the AL, ORNs synapse onto two primary olfactory center neurons, projection neurons (PNs) that leave and drive higher order brain centers, and local interneurons (LNs) which remain within the $A L$ and form primarily inhibitory and modulatory connections with ORNs and PNs. One function of the local circuitry is to mediate gain control to presented stimuli through GABA mediated lateral inhibition to ORNs (Olsen and Wilson, 2008), though glutamate and the neuropeptide tachykinins also mediates lateral ORN inhibition (Ignell et al., 2009; Liu and Wilson, 2013). Further modeling shows that intraglomerular interactions selectively amplify weak inputs whereas interglomerular interactions inhibit or normalizes the responses to odors leading to a decorrelation of PN response patterns (Olson et al., 2010). 
As mentioned, output of the $\mathrm{AL}$ from PNs follow three main tracts. The medial antennal protocerebral tract is the largest of the three. It projects information to the calyx of the mushroom bodies eventually terminating in the lateral horn. The lateral protocerebral tract takes the opposite trajectory, where the tract initially sends axon collaterals to the lateral horn and then terminate in the mushroom bodies. Within the mushroom bodies, PNs synapse onto multiple intrinsic Kenyon cells, which show sparse response profiles to odors (Perez-Orive et al., 2002). In the lateral horn, PNs synapse in a distinct spatial pattern. Projection neurons from a given glomerulus project to the same area in the lateral horn (Wang et al., 2002); with distinct lateral horn cell types receiving different combinations of glomerular input (Jeanne, et al., 2018). This suggests a conserved spatial map within the lateral horn; however, the response properties of protocerebral cells is most likely affected by the temporal structure of PN activity.

To ensure proper responses in a variety of contexts, nervous systems must be flexible to changing behavioral demands. Therefore, extrinsic modulatory systems ensure that the system responds optimally under different behavioral contexts. Insect extrinsic modulation is mediated by aminergic innervation by cells releasing octopamine, serotonin, dopamine (Mercer et al., 1983) and histamine (Homberg, 1994), as well as several neuropeptides (most of which originating from intrinsic antennal lobe sources). In the antennal lobe, octopamine has been shown to enhance learning and memory through the VUMmx1 neuron who is driven by sucrose response cells (Hammer and Menzel, 1995; Hammer and Menzel, 1998) (present in Manduca (Dacks et al., 2005)). Dopamine upregulates neural activity through cAMP (Beggs and Mercer, 2009) and facilitates aversive learning (Dacks et al., 2012). However, the role of histamine modulation remains unclear. 


\section{Histamine}

Beta-iminazolylethylamine was first synthesized by Windaus and Vogt in 1907 (Dale and Laidlaw, 1910). Histamine, the common name, results from the decarboxylation of the amino acid histidine by histidine decarboxylase dependent on a pyridoxal 5' phosphate (Burg et al., 1993). Histidine decarboxylase is highly conserved across the animal kingdom (Burg et al., 1993; Haas et al., 2008) and histamine shows many effects on animals from insects to molluscs to humans (Haas

et al., 2008). In vertebrates there are 4 known histamine receptors found across the periphery and the central nervous system. Following histamine receptor binding, histamine detaches from the receptor and is metabolized via enzyme mediated methylation or glial cell mediated recycling (Haas et al., 2008).

In the insect nervous system histamine plays a significant role in the visual system; primarily by transmitting information through photoreceptor release onto large monopolar cells (LMCs) in the lamina of the fly retina. In this scenario, histamine plays the role of glycine in the mammalian retina where darkness leads to constant excitation of post-synaptic LMCs and is inhibited upon photoreceptor exposure to light. Here, histamine acts as an inhibitory neurotransmitter mediated by two identified histamine receptors. Arthropod histamine receptors are ligand gated $\mathrm{Cl}$ channels (McClintock and Ache, 1989; Hardie, 1989) sharing 45\% amino acid similarity to the alpha 3 subunit of the human glycine receptor (Zheng et al., 2002). The Drosophila histamine decarboxylase (hdc) gene shows $62 \%$ similarity to the human hdc gene with $90 \%$ amino acid sequence homology, including several potential phosphorylation sites. In both the meso and metathoracic neuromeres, there exist a pair of ascending histamine neurons to the brain, with additional pairs of in the abdominal ganglia that do not project to the brain. In addition, there are ten bilaterally projecting pairs of histamine cells in the midbrain and one in the subesophageal ganglion with no labeling in the protocerebrum, mushroom bodies or lateral horn. Additionally, there are histamine cells that have evolved in particular insects; for example, histamine 
immunoreactive local interneurons in the AL of hymenoptera (Dacks et al., 2010). The goal of this first study was to characterize the ascending pair of histamine immunoreactive neurons to the deutocerebrum in Manduca sexta. Additionally, we show histamine receptor B immunoreactivity in the $A L$ and its co-localization with the local interneuron neurotransmitter GABA and neuropeptides tachikynin, allatotropin and FMRFamide.

\section{Methods}

To accomplish the goals of this study, we used immunohistochemistry to identify the distribution of histamine cells in the nervous system. In this method the antigen, histamine, is injected into the bloodstream of another animal (here rabbit). Because histamine is normally present, it must be modified to engage the immune system, this is achieved by linking histamine to carbodiimide. Following injection, the animal is bled and the antibody against the injected antigen is isolated. The isolated antibody is applied to an extracted and fixed nervous system tissue. A series of washes and sectioning of the tissue at approximately $100 \mu \mathrm{m}$ preceded incubation of the tissue in the purified antibody. After a couple of days, the tissue was washed and a secondary antibody targeting the original antibody and who is tagged with an excitable fluorescent molecule. Tissue is then mounted on a microscope slide and imaged on a laser scanning confocal microscope. Image stacks are further analyzed in the Olympus Fluoview software, Corel Draw, Adobe Photoshop and Vaa3D software for image reconstruction. Given our previous knowledge of histamine innervation of the antennal lobe, we hypothesized that one or both of the histamine receptors would be expressed in antennal lobe tissue. Using rtPCR on extracted antennal lobes we identified weak histamine $B$ receptor expression in this tissue. Because we did not see histamine A receptor expression, a polyclonal antibody against the Manduca sexta histamine B receptor was made. Western blot and pre-absorption assays were performed to confirm the specificity and legitimacy of the antibody. Receptor immunolabeling showed characteristic 
histamine receptor immmunolabeling in the lamina of the optic lobe with no labeling when preabsorbing the antibody to the target peptide sequence. Finally, a single band was seen in western blot analysis; together, this information suggests that the specificity against the peptide sequence from the histamine receptor is exclusive. Manduca larval nervous systems were also analyzed for the MDH neurons with methodology similar to that described above. An additional study was performed where we cut the ventral nerve cords in moths to ablate/kill the axons of the histamine cells and left for 6 days. Here, we sought to verify the thoracic ganglia origination of the histamine projections to the antennal lobe.

\section{$\underline{\text { Results }}$}

Two large histamine immunoreactive neuron cell pairs were found in each the mesothoracic and metathoracic neuromeres. These cells send a large primary neurite dorsally and medially before turning orthogonally and projecting to the lateral part of the ganglion. Projections extend anteriorly through the prothoracic neuromere and neck connective towards the subesophageal ganglion and brain, terminating in the deutocerebrum. This conclusion was validated by performing a set of "lesion experiments". First, the ventral nerve cord was severed; second, the metathoracic neuromeres were sectioned from the rest of the CNS in vivo approximately 6 days prior to immunolabeling. This work showed no remaining labeling in the antennal lobe following sectioning above mesothoracic neuromere but not below it indicating that the HA cells that ramify the AL are in the mesothoracic neuromere. The wide projecting fibers within the mesothoracic ganglion suggests that these cells receive a variety of inputs and the blebby nature of the more lateral processes within the mesothoracic ganglion suggests that histamine may be released locally in the thoracic ganglia though this remains to be confirmed. 
To further understand the nature of the MDH circuit, we examined the immunoreactivity of the histamine B receptor in Manduca antennal lobes. We confirmed the selective expression of the histamine B receptor in the antennal lobe using RT-PCR (and found no band for the histamine A receptor). Our results demonstrate widespread histamine B receptor expression throughout glomeruli in the antennal lobe. Given the widespread distribution of GABA immunoreactivity in the antennal lobes we co-labeled antennal lobe tissue for the MsHisCIB and GABA and found a subset of these GABAergic cells also labeled for the histamine B receptor. Additionally, we considered the co-immunolabeling of MsHisCIB and the neuropeptides FMRFamide and allatotropin. We found that one local interneuron for each allatotropin and FMRFamide co-labeled with the histamine B receptor. Together this suggest a complex functionality of this circuitry.

Finally, in Manduca larvae, the MDH neurons appear to be present with clear labeling of a pair of histamine cells in the thoracic ganglia and projections all the way to the larval antennal center. However, there is no evidence of MsHclB receptor labeling in the larval antennal center, and hence there is no complete circuit. This suggests that the MDH cells in the larval nervous system serve a different function, assuming that they have a larval function, which has yet to be established.

\section{Conclusion}

The current study intended to characterize a histamine immunoreactive circuit in the moth Manduca sexta. Here we found that there was a pair of large cells that originated in the mesothoracic ganglia which houses the flight sensory motor centers. These cells project to the deutocerebrum and terminate in the antennal lobe and the antennal mechanosensory and motor centers. We found that the circuit is complete given histamine B receptor expression in antennal lobe tissue. Furthermore, the 16 neurons appear to remain local to the antennal lobe and are 
predominantly inhibitory as all but one co labeled for GABA suggesting that one consequence of activation of the MDHns is suppression of an inhibitory network within the antennal lobe. Together these results suggest that Manduca sexta uses information from the flight sensory motor centers to disinhibit information processing in the $A L$, most likely during odor guided flight. Subsequent studies were performed to first evaluate the generality of this circuit across the insects and then to adequately address the functional/computational role of this circuitry and what this type of circuitry means for active sensing modalities. 


\section{Chapter 2}

The information here is a summary of the full research article published by Chapman et al., 2018 in the Proceedings of the National Academy of Sciences; for more information on the methodology and results of the study see Appendix B. In this study, I personally performed the experiments where we "broke" the histamine circuit by severing the ventral nerve cord or bath application of histamine receptor antagonist, cimetidine. Finally, I facilitated the histamine circuit's function by bath applying histamine to the antennal lobe. 


\section{Introduction}

\section{A brief history of motor to sensory circuitry}

Perceptions of the world are often believed due to sensations driven by stimuli originating in the environment. However, agents in the world are not static. Movement through the environment distorts the sensory landscape and, not surprisingly, the motor centers driving these movements affect the relevant sensory system. Historically the notion of action guiding perception dates to pre-Socratic thinkers who believed that the eye emits light which is subsequently reflected back to the eye which then results in a perception. In the 19th century, George Steinbuch provided the first description of a behavioral process that required motor innervation. He believed that the ability to tactically identify objects depended on the motor signals that governed reaching and grasping (Grusser, 1995).

While the prominent psychologist and philosopher William James believed an important role for motor circuits affecting perception, a detailed explanation from neuroscientific terms was not until a review by Von Holst and Mittelstaedt (1950). In this review the authors were interested in reinterpreting experiments widely believed explained by the dominant theory of the time, the reflex hypothesis. Here sensory information activates sensory receptors which drive a chain of neural responses ending in a behavior. Inherent in this theory was the constant dependence of behavior with the environment despite many counterexamples of persistent behavior following sensory system decoupling. Von Holst and Middlestaedt believed that an efference copy signal of an action command signal is copied and sent to the affected sensory system where it interacts with the incoming reafferent stimulus. 
More generally, circuits of this nature are called corollary discharge circuits and are found in many animals (Crapse and Sommers, 2008). In the following, we will discuss a diversity of neural processes that use corollary discharge, starting by explaining very simple efference copy circuits that directly inhibit the activity of specific sensory cells; in particular reflex inhibition in Caenorhabditis elegans and auditory filtration in the cricket. Second, we will examine saccadic eye movement corollary discharge circuits in fruit flies and rhesus monkeys, and finally we will look at other higher order corollary discharge circuits that govern learning in songbirds and gain modulation in weakly electric fish.

\section{Efference copy circuits mediating reflex inhibition and auditory filtration}

A simple but elegant example of a corollary discharge circuit is found in crickets. Crickets (and katydids) use stridulation to generate auditory signals for communication; a trait believed to date back 150 million years ago, with 2000 current species. Song function varies from calling and courtship behaviors to territorial and aggressive songs between males (Alexander, 1962). To produce the sounds necessary for the song, crickets open and close their wings while scraping them together. During the closing of the wings, a pick like structure (plectrum) on the left-wing brushes over a comb like structure (file) on the right-wing producing syllables of vibrations with a characteristic frequency. These vibrations cause the harp (or mirror in katydids) on the wing to resonate and amplify the songs (Alexander, 1962; Jordan et al., 2010). The motor patterns and muscles required to produce opening and closing are controlled by descending signals from the brain that drive rhythmic motor patterns to produce species specific songs (Elliott, 1983; Hedwig, 2000). This adaptation of song production, however, came with a cost. If the cricket is singing the intensity of the sound it produces ( $100 \mathrm{db}$ at the harp) will overwhelm the auditory system making it challenging to hear other sounds emanating from the outside world. In order to maintain auditory sensitivity to its surroundings, a copy of the motor signal drives a single neuron, corollary discharge interneuron (CDI), that projects to and effectively and selectively filters out the ability 
for auditory cells in the prothoracic ganglion to transmit auditory stimuli produced by stridulation (Poulet and Hedwig, 2002; 2003; 2006). In doing so the crickets auditory system remains acute to other relevant sounds. The CDI neuron has extensive branching in each of the thoracic and abdominal ganglia as well as the brain, suggesting that it may influence other behaviors as well (Poulet and Hedwig, 2006); though here a single neuron is responsible for optimizing sensory processing in the context of a behaving animal.

Sensory filtration systems like this are found in some of the simplest nervous systems. For example, the roundworm Caenorhabditis elegans has 302 neurons (White et al., 1986) but nevertheless shows a forward circuit, which predicts the occurrence of a reafferent stimulus and suppresses behavioral responses to it. These worms have a reflexive escape response to contact at either the anterior or posterior end of the worm (Chalfie et al., 1985). However, movement should activate mechanosensors at the leading end, and hence the worm should be perpetually moving back and forth with continuous activation of the reflex response. A corollary discharge circuit projecting from the neural centers driving movement inhibits the escape response reflex and hence the worm continues forward (Chalfie et al., 1985; Oulette et al., 2018). These examples show simple neural circuits whose function is to optimize sensory processing by selectively inhibiting reafferent stimuli. However elegant a solution these examples show, there are other corollary discharge circuits whose function is more complex.

\section{Corollary discharge circuits mediating saccades and visual processing}

Reafferent stimulus cancellation is more complex in drosophila saccadic eye movements. Unlike vertebrates who use oculomotor behaviors that shift the retinal eye field location, Drosophila and other insects move their heads to shift their gaze (Collett and Land, 1975; Bender and Dickenson, 2006). While flying they rotate their heads and bodies in the yaw, pitch and roll planes, and as such are able to view more of the world. A recent study dissected the role of the horizontal and 
vertical systems in the drosophila retina. Here three distinct subsets of cells respond to optic flow stimuli. The horizontal system is comprised of 3 cells on each side, and the vertical system has 6 cells separated into three groups (Scott et al., 2002). Each of the three groups responds to a different plane of rotation. Motor signals driving yaw movements innervate Hs cells, roll is Vs(13) cells, and pitch is Vs(4-6) (Schnell et al., 2010; Kim et al., 2015; 2017). Saccadic head movements in the yaw plane show suppression of optic flow responses in Hs cells with medium suppression of $\mathrm{Vs}(4-6)$ with almost no suppression to the pitch detecting $\mathrm{Vs}(1-3)$ cells (Kim et al., 2017). Collectively, these cells optimize visual processing with body movement specific innervation of motion sensing cells in three dimensions. Similar saccadic suppression circuits exist in other insect species as well (Zaretsky and Rowell, 1979).

Saccadic eye movements in primates also serve as an example of a higher order corollary discharge circuit (Crapse and Sommer, 2008), where movement detecting cells aren't simply inhibited during motor movements (though they do (Bremmer et al., 2009)). Rhesus monkeys use saccadic eye movements to scan a scene; however, in addition to filtering out potential optic flow information, they optimize information processing while scanning a scene by predicting the spatial location of focus. When doing so, a corollary discharge circuit projects from the superior colliculus, an area known for controlling ocular motor behaviors, to the frontal eye field, an area known to control visual sensitivity in a retinotopic fashion, and projects through the thalamus (Sommer and Wurtz, 2002;). The circuit shifts the frontal eye fields selective attention to the future field immediately prior to eye movements to the target (Colby and Goldberg, 1992; Umeno and Goldberg, 1997). Disrupting this thalamic circuit causes a disruption of visual responsiveness in frontal eye field neurons (Sommer and Wurtz, 2006). This suggests that optimal visual processing in the context of saccadic eye movements is predicated on a corollary discharge circuit that updates the visual processing centers in anticipation of a change in visual attention, however the behavioral consequences of this disruption is not known. 


\section{Corollary discharge circuits mediating learning and gain modulation}

An efference copy circuit has been proposed in learning motor movements in the cerebellum with a similar learning mechanism also seen in birdsong learning. Songbirds, such as canaries and zebra finches, sing to communicate. Young male birds learn the song of their fathers and/or nearby conspecific males. To learn a new song, birds complete an overlapping, two stage process. The first process is referred to as imprinting. In this stage young birds listen to fellow adult birds and in the process the brain encodes the song. The second stage, the bird attempts to vocalize their song and in the process compares what he sung to the template copy of the song imprinted in the nervous system (Brainard and Doupe, 2000). In order to accomplish this task, one group has proposed a corollary discharge model to overcome delay constraints inherent in auditory feedback models. Here motor centers that drive song production send an efference copy signals to cells that project to sensory centers who process auditory feedback information (Troyer and Doupe, 2000).

Relevant to the current study is a corollary discharge circuit which enhances sensory processing during active sensing processes. One circuit that appears to have some of these features is the electrosensory system in weakly electric fish. These fish take advantage of the conducting medium of water to emit and receive electrical signals that serve as a means to navigate their environment and communicate with other members of the species. These fish have specialized electroreceptors which transduce electrical signals in the water and project to the electric lateral line lobe for processing. The sensory receptors that project to the electric lateral line lobe synapse onto efferent cells that project information to other brain regions, and inhibitory Purkinje like cells (MG). These species have been model systems for many things, not least of which is to study corollary discharge circuits. The command signals that generate the electric organ discharge are copied and sent to the electric lateral line lobe to inform the sensory system of the previous discharge. The role of this corollary discharge is believed to be two-fold. One is to distinguish 
signals generated from other sources and reafferent signals (Bell, 1981; Crapse and Sommers, 2008). The second is to selectively amplify and or degrade electrical signals in different cell types within the electric lateral line lobe. Experimenters found differential responses to corollary discharge signals with type I MG cells showing an excitatory response, and type II MG cells showing an inhibitory response. This corollary discharge circuit shows a different effect than others found in the animal kingdom but may represent the closest comparison to the proposed studies (Mohr et al., 2003) in that the consequence of corollary discharge and have a net upregulation in a behavioral context.

Here we demonstrate an enhancement of reafferent stimulus response in the olfactory system of the moth Manduca sexta that is mediated by a corollary discharge circuit emanating from flight sensorimotor centers. In particular, we show that the previously described circuit shows tonic activation, and whose firing rate is correlated with fictive flight behavior. This input to Manduca's olfactory system modulates the ability to process high frequency natural stimuli encountered during odor guided flight as pharmacological and surgical disruption of this circuit decreases the ability to track rapidly pulsed odors. This disruption also decreases olfactory acuity as measured by psychophysical assays of detection and discrimination.

\section{Methods}

All studies were performed on Manduca sexta adults between 4 and 7 days eclosion. First, to determine the relationship between wing motor function and the histamine cells, whole cell recordings were made with sharp electrodes in intact animals from the dorsal side of the mesothoracic ganglion with $\mathrm{MDH}$ identity determined by dye injection at the recording site. Simultaneously, suction electrode recordings of nerve IIN1b that controls forewing elevator and depressor muscles. Recordings were performed in the presence of chlordimeform an octopamine 
agonist, which activates flight central pattern generators and hence is termed fictive flight. Spike trains were analyzed in Spike2 and Matlab software.

Next, to determine the impact of the histamine cells action on antennal lobe function, antennal lobe recordings were performed using multi-unit electrodes in whole body preparation. Odors used in this study were the ketones hexanone and octanone. Manduca has previously been shown to be able to discriminate these odors and analysis of population responses show significant differences in population activity. Odors were presented continuously and as pulse trains during trials preceding and following one of three treatments; application of a histamine receptor antagonist or severing of the neck connective including the $\mathrm{MDH}$ neuron axons to disrupt histamine function, or conversely by application of histamine. Pharmacological treatments were later washed with physiological saline to determine if any effects could be rescued. In ablation studies, moths were again presented with continuous and pulsed stimuli, this time before and after severing the ventral nerve cord. Nerve cord ablations were performed blindly and approximately half the experiments served as controls where the ventral nerve cord was not cut (sham cut experiments). Multi-unit spike trains were sorted offline using MClust software and further analyzed in Matlab for their ability to entrain to temporally structured stimuli.

Finally, to determine the consequence of histamine function on olfactory acuity, behavioral studies assaying olfactory detection and discrimination threshold were performed on moths trained using the same odors as above using Pavlovian conditioning paradigm. Restrained moths were presented with an odor followed by a sucrose reward. Moths have the ability to learn odors, and trained odors served as instruments to measure detection and discrimination thresholds. Moths were tested using an ascending dilution series such that detection thresholds could be determined by statistical comparison with responses to blank odor cartridges. Animal antennal lobes were 
either pico-injected with inert saline as an injection control or saline with the histamine receptor antagonist cimetidine.

\section{$\underline{\text { Results }}$}

The mesothoracic ganglion houses the neurons responsible for the central pattern generators that drive the patterned wingbeat behavior, proprioceptive neurons that respond to wing displacement, and motor neurons that drive muscle contractions responsible for flight. The MDH neuron is an interneuron with large diameter cell bodies sitting on the dorsal side of the mesothoracic ganglion. MDH cells shows tonic spontaneous activity with an increase in firing rate in response to chlordimeform application that is correlated with a concurrent increase in nerve afferent IIN1b.

Antennal lobe population responses can discriminate closely related odors. Additionally, the biophysical properties of at least a subset of cells allow bursting firing patterns at high frequencies consistent with reafferent stimuli experienced during odor guided flight. Severing the ventral nerve cord showed a reduction in this ability as measured by power spectral density. Furthermore, application of histamine increased pulse tracking fidelity while the histamine receptor antagonist cimetidine reduced pulse tracking ability. These results are consistent with the hypothesis that the mesothoracic to deutocerebral circuit facilitates the processing of natural stimuli. Finally, psychophysical measures of olfactory detection and discrimination show that more odor is necessary when the histamine circuit is blocked suggesting that the functionality of this circuit is behaviorally relevant to the animal in that it normally enhances sensitivity presumably during flight. 


\section{Conclusions}

The preceding set of experiments represent the first detailed characterization of the physiological consequences of a corollary discharge circuit an olfactory system. Additionally, there are two pieces of evidence to suggest that this would be the first higher order corollary discharge circuit in an invertebrate. First, the response of the MDH neurons to fictive flight is tonic. If this system's function was to filter out the periodic reafferent stimulus, one would expect the response would also be periodic and synchronized to the wing beat. Second, disrupting this circuit through ablation and pharmacological means disrupts antennal lobe processing of the reafferent stimulus. Along with histamine bath application's up-regulation of this ability, it appears this system is exploiting the periodicity induced by wing beating to enhance odor-guided behavior; this is consistent with increased detection thresholds in subjects whose antennal lobes are pico-injected with the histamine antagonist cimetidine. 


\section{Chapter 3}

The information here is a summary of the full research article published in Chapman et al., 2017 in Proceedings of the Royal Society B; for more information on the methodology and the results of the study, see appendix C. In this study, I personally performed the immunolabeling for $M$. sexta, P. rapae, B. mori, P. appalachiensis, L. archippus, G. molesta, G. mellonella, G. lurida, T. molitor, and caddisflies. 


\section{Introduction}

\section{Insect Ecology}

To understand how the nervous system functions, it is necessary to understand the behaviors and behavioral drivers of the organism. These behaviors are shaped by the collection of food resources, oviposition sites and mates available to the individual with remarkable examples of evolved traits. Flowering plant evolution and success was dependent on the presence of insects who provide pollination in exchange for sucrose and amino acids. There exist multiple examples of coevolution between plants and insects with unique behaviors and morphologies to support their interactions.

A dramatic example of this is the Madagascar sphinx moth whose proboscis extends $30 \mathrm{~cm}$ in order to get to the pit of Angraecum sesquipedale an orchid with a foot-long nectary (Kritsky, 1991). In this case, the flowers provide nectar to animals who are large enough to pollinate the plants, and large insects, particularly those who hover, require large amounts of energy and therefore seek flowers that have large amounts of nectar (Price, 1997). Another example is the mating behavior of two butterfly species of the genus Heliconius. Heliconius cydno has iridescent wings which reflect mostly polarized light, while Heliconius melpomene does not have iridescent wings and does not reflect polarized light. In a clever experiment using various light filters, butterflies exposed to female $H$. cydno, shows reduced mate approach behavior in $H$. cydno compared to $H$. melpomene when the filter blocked polarized light. This suggests that reflected polarized light drives mating behavior in these butterfly species (Sweeney et al., 2003).

\section{Behavioral differences between moths and butterflies}

Nervous systems cost an enormous amount of energy to maintain, and hence efficiency in neural architecture is a significant evolutionary constraint. If a given neural center is large, it is reasonable 
to assume that the given neuropil processes information very relevant to the animal. One example of this is the pheromone system in moths and other insects. Here, moths and other species that rely heavily on pheromones for mating behavior, devote a significant portion of their olfactory systems to process these chemicals. Other species such as butterflies that rely less on pheromones, have smaller regions dedicated for pheromone processing. These two groups of Lepidoptera also show dramatic differences in flight behavior. Manduca shows rhythmic and characteristic scalloping while it is flying through the environment. Here they beat their wings a few times as they thrust forward and upward and glide as they drift lower toward the ground (Stevenson et al., 1995). Butterflies comparably fly quite chaotically with less rhythmic wing beating behavior.

Interestingly, there are at least two moths who show stereotyped behaviors consistent with Manduca's, Bombyx mori and Grapholita molesta. Bombyx mori is a model species of moths who make silk. Humans have selectively bred these moths to maximize silk production and in doing so has made flight impossible. However, when approaching a female calling with pheromones, they will still continually beat their wings until they reach their target on foot. In fact, removing the wings so that air disturbances caused by the wing beating are absent while leaving locomotion intact, eliminates the moth's ability to find their target (Obara, 1979). Grapholita molesta is a relatively distantly related moth that flies through its environment tracking pheromone plumes; however, it mates with females on a tree branch, and while tracking on foot continues to beat its wings. These examples show a robust behavior in the absence of an apparent functional need suggesting that the behavior of these animals is important to their functioning in ways other than their primary function (i.e. flight), most likely, by altering the sensory experience. In the context of the present study, one would think that the neural circuitry responsible for processing this type of information would be conserved across species solving a common problem while being lost in the 
more closely related butterfly species, which have different behavioral ecologies and hence different demands on the nervous system.

\section{Comparison of peripheral olfactory system appendages}

Some of the most apparent species-specific differences in olfaction stem from specific differences beginning in the olfactory periphery. Differences in antennal morphology are apparent across different insect species, whereas some species of the Lepidoptera have extravagant antennae, Drosophila's antenna (for example) is quite small. Insect olfactory receptor neurons can express multiple receptor types in a single cell, and most olfactory receptors have the co-receptor Orco that is also expressed. Without a functional Orco protein, the receptor neuron does not show normal responses to odors (Krieger et al., 2003; Larrson et al., 2004). However, one species, the Hessian fly Mayetiola destructor shows different response properties to Orco natural ligands and forms non-functional receptor complexes in Drosophila (Corcoran et al., 2018) suggesting a possible diversity in orco function. While there are ionotropic receptors in the insect olfactory system, most ORNs express receptors of a large family of proteins that are similar to G-protein coupled receptors (Robertson et al., 2003).

As mentioned in Chapter one, olfactory receptor neurons can show a wide range of response profiles and tunings. The species Drosophila sechellia lives on the island of Seychelles off the eastern coast of Africa. This species shows an appetitive behavior to the odors of the morinda fruit, whereas other species of drosophila have an aversive response. Dopamine is a necessary chemical for laying eggs and sechellia flies have low levels of dopamine because of a genetic mutation. The morinda fruit shows high levels of L-DOPA which when consumed increases female dopamine levels (Lavista-llanos et al., 2014). In this fly's antenna an entire sensillum has been devoted to receptor cells expressing a receptor which only responds to an odor specific to the morinda fruit. Given the close relation to Drosophila melanogaster, this represents a unique 
specialization in this drosophilid species (Dekker et al., 2006). Examples of more rapid evolutionary change in the chemosensory periphery exist in German cockroaches. In the recent past humans have built traps to kill cockroaches in their house using sugar as the appetitive stimulus for the roaches. Within a quick evolutionary time frame these species show a response in receptors that normally respond to the bitter chemical caffeine become responsive to glucose leading to an aversive behavior from sugary food sources (Wada-Katsumata et al., 2013).

\section{Comparison of antennal lobes and higher order processing areas}

In addition to the olfactory periphery, the central olfactory system also can show dramatic differences in structure and size. Again, given the high metabolic cost of nervous system tissue, antennal lobe sizes differ with moths and bees having rather large olfactory systems, and dragonflies have greatly reduced olfactory processing centers, though they do display odordependent behaviors during prey capture (Piersanti, et al., 2014). Within the antennal lobes there are species specific differences in local circuitry, with projection patterns to higher order processing centers, and centrifugal modulation projecting to it. Local interneurons also differ in the neurotransmitter content (excitatory or inhibitory) and in their branching patterns. Intrinsic and extrinsic modulatory cells have complex innervation patterns in both the glomeruli they innervate and the pattern of innervation therein. For instance, in Manduca different modulatory systems may synapse onto different neural subtypes, with serotonin cell morphologies suggesting modulation of projection neurons and local interneurons, while dopamine cell morphologies suggesting modulation of projection, local and receptor cells (Lizbinski et al., 2016). These differences represent unique adaptations to optimize sensory processing in the context of ethological pressures.

There are three major output tracts from the antennal lobe to the lateral horn and mushroom bodies. These tracts also show species differences where in many insect species, the lateral tract 
does not terminate in the mushroom bodies like they do in moths, bees, flies, and ants (Martin et al., 2011). The lateral antennal protocerebral tract in honeybees has been shown to be a parallel processing example along with the medial antennal protocerebral tract (Rossler and Brill, 2013). In this study the experimenters showed that biologically relevant odors caused activity in each tract; however, the medial antennal protocerebral tract show narrow tuning responses to odors and the lateral antennal protocerebral tract show broad tuning profiles, suggesting each tract may have separate functions (Rossler and Brill, 2013).

A recent interesting example of a species-specific environment-driven neural pathway was found in Drosophila melanogaster. In addition to having a dedicated pathway for pheromones, they have dedicated olfactory receptor neurons tuned to the molecule geosmin which is produced by fungi and bacteria. These olfactory receptor neurons respond strongly and selectively to geosmin and then projects to an individual glomerulus in the antennal lobe. This is the only known odor to which this glomerulus responds and then leaves the AL projecting to the mushroom body calyx and lateral horn. This molecule is known as a strong innate avoidance molecule for drosophila and is so much so that it has a dedicated circuitry found across most drosophilid species (Stensmyr, et al., 2012). These examples show how the olfactory nervous system is evolutionarily plastic to environmental and species-specific demands. In this vein, the following comparative study was intended to examine the phylogenetic distribution of axon projections of the histamine immunoreactive neurons in insects and particularly the Lepidoptera and show a relationship between behavioral repertoire and evolutionary changes in neuroanatomic circuitry via co-option.

\section{Methods}

The animals used in this study were Manduca sexta, Bombyx mori, Idia aemula, Papilo appalachiensis, Limenitis archippus, Pieris rapae, Theatops californiensis, Grapholita molesta, 
Galleria mellanella, Gyna Iurida, Tenebrio molitor, Trichoptera, Drosophila melanogaster, and Amblyomma americanum phylogenetically extending 250 million years. Within Lepidoptera, we had four moth species and three butterfly species with an outgroup of Trichoptera (caddisflies). Among the four moth species Bombyx and Manduca are macrolepidoptera while Galleria and Grapholita are microlepidoptera; this is significant because the macrolepidoptera moth species are phylogenetically more closely related to butterflies than to the microlepidoptera species.

To determine the anatomical structure of a pair of histamine immunoreactive neurons in the antennal lobe, we used immunohistochemistry. We used a rabbit anti-histamine antibody to identify histamine localization within the brain and thoracic ganglia of each species, and a mouseanti bruchpilot antibody to identify glomeruli of the antennal lobe. The protein bruchpilot is found at all synapses in the drosophila brain; given the high synaptic density of glomeruli, labeling outlines neural structures including the antennal lobe and its glomeruli. Each antibody was tagged with a second immunoflourescent antibody. Brains were imaged on an Olympus laser scanning confocal microscope. Images were analyzed in Fluoview software, Corel Draw and Adobe Photoshop.

\section{$\underline{\text { Results }}$}

Immunohistochemical examination of histamine showed conservation of the pair of MDHns in the mesothoracic ganglia that ascend to the brain. The MDHns were observed in all species studied, with the exception of ticks, suggesting that this circuitry has ancient origins in arthropods. Antennal lobe innervation by the MDH neurons on the other hand, was restricted to moths and caddisflies. Given the absence of histamine labeling in the antennal lobes of butterflies and its presence in microlepidopteran moths and caddisflies suggests this circuit has lost its function in butterflies most likely due to lack of necessity or demand. The absence of MDHn projections to 
the antennal lobes of all other insect species studied but the persistence of projections to other brain regions suggests that the MDHns have been co-opted for an olfactory function in night flying and plume tracking insects.

\section{Conclusion}

Understanding the phylogenetic relationship between nervous system structures and behaviors can elucidate neural processing principles. Given the metabolically demanding nature of nervous system tissue, circuitries not providing behavioral advantages are often lost or restructured to serve a different purpose. Here we showed that a histamine immunoreactive circuit, connecting flight sensory motor centers in the mesothoracic ganglia to the brain, exists in distantly related arthropods. Additionally, we showed that this circuits' morphological innervation of the AL was restricted to night flying, plume tracking moths and caddisflies, but not in more visually guided, day flying butterflies. Together these findings suggest a conserved circuit whose detailed projection patterns depend on the animal's behavioral repertoire. 


\section{Discussion}

The studies described in this dissertation have demonstrated a neural circuit projecting from the flight sensory motor centers in the mesothoracic ganglion to the deutocerebrum, including the antennal lobe whose disruption leads to deficient odor processing and behavior. Motor to sensory circuits are often discussed within the context of internal models or corollary discharge circuits. The results we obtained herein describe a structurally and functionally novel corollary discharge circuit. For one, current invertebrate models have not shown a corollary discharge circuit one would classify as higher order (See Crapse and Sommer, 2008). Lower order corollary discharge circuits are like the examples described in Chapter 2 where reafferent information is filtered out through efference copy signals. Higher order corollary discharge circuits are believed to enhance more complicated cognitive tasks such as sensory stability (as seen in Rhesus monkey saccades) or learning (songbirds). While higher order corollary discharge circuits have not been characterized in invertebrates, it is worth pointing out that dragonflies may have a circuitry mediating prey capture (Mischiati et al., 2015). Second, our example shows an enhancement of processing natural periodic stimuli. Previous vertebrate models have not shown something akin to this type of mechanism. On the one hand, it appears as though the motor system is predicting the occurrence of temporally structured stimuli, but it uses this information to sharpen the periodic response by increasing the power of $A L$ neuron responses at $20 \mathrm{~Hz}$.

The functionality of any neural circuit is due to many interacting factors; one being the nature of the receptors binding the released neurotransmitter. Insects possess two histamine receptor types, HisClA and HisClB (Gisselman et al., 2002, Zheng et al., 2002), both of which are ligand gated chloride channels (McClintock and Ache, 1989; Hardie, 1989). Each receptor is homomeric with two genes coding for two subunits $\mathrm{HisCl}$-aplha1 and HisCl-alpha2 (Gisselmann et al., 2002). These receptors are members of the large cys-bridge superfamily of ligand-gated ion channels 
comprised of four transmembrane domains (Gisselmann et al., 2002). This is in direct contrast to the four histamine receptors in vertebrates that are seven transmembrane domain G-protein coupled metabotropic receptors.

His CIA and His CIB receptors have physiological differences in their response properties, as well as their role in processing sensory information. Transfected S2 cells with homomeric HislA receptors shows lower sensitivity to bath applied histamine than homomeric HislB receptors or heteromeric receptors with much more sensitivity seen with heteromeric receptors (Pantazis et al., 2008). In knockout flies for HisCIA receptors electroretinogram ON/OFF responses were abolished, whereas in HisCIB receptor knockouts retinal responses were increased (Pantazis et al., 2008; Yusein et al., 2010), while wild type flies, in the presence of ivermectin (a histamine B receptor agonist) showed an increase in peak amplitude and onset latency of the OFF response (Yusein et al., 2008). Given the anatomical segregation of receptor expression patterns of these two receptors, it is not surprising that they show different response profiles to light. As of now it is unclear what the computational effect of HisCIB receptors have on light processing. However, it may be involved in gain control of photoreceptor responses to light (Kupenova and YuseinMyashkova, 2012); where this could be affected in various ways such as histamine concentration in photoreceptors or ion distributions (see discussion above). In R7 and R8 photoreceptor cells, His CIB receptors appear to mediate opponent processing of color through $R 7 / R 8$ reciprocal inhibition (Schnaitmann et al., 2018). On a longer timescale, it appears as though the HisCIB receptors on $\mathrm{R} 7 / \mathrm{R} 8$ photoreceptors, along with HisCIA receptor activity, is also sufficient to synchronize rest/wake cycles with light dark cycles (Alejevski et al., 2019). Additionally, it is reasonable to question whether the responses of histamine receptors are sensitive to any other endogenous ligands. In the butterfly Papillo xuthus both HisClA and His CIB receptors showed physiological responses to GABA as well as histamine. While the effect of GABA was on the order of 100 times less sensitive, the synergistic effect of both GABA and histamine was significantly 
stronger than to GABA or histamine alone (Akashi et al., 2018). Given the widespread distribution of GABA throughout the nervous system including the lamina, the effect and computational consequences of this complex system is unclear.

Pulse tracking does not occur on a spike by spike basis but rather each 'ON' epoch corresponds to multiple spikes referred to as a burst. From a computational perspective these bursts relay information in a more reliable fashion with increased neurotransmitter release per cycle. Analysis of Kenyon cells in the mushroom bodies show sparse responses and high projection neuron to Kenyon cell convergence (Perez-Orive et al., 2002). If the projection neurons transmitting this information are both bursty and coherent (at least for some duration of the burst cycle), then the reliability of the responses of these cells would be increased particularly at lower concentrations. This would also be consistent with the effect of pulsing odors decreasing false positive rates (Daly et al., 2013).

While the function of the Manduca's MDH circuitry is difficult enough, what purpose these cells play in other insects is even more fleeting. What purpose would wing sensory-motor information be for the midbrain of the butterflies? Though no answer will be given, the "supermodel" system of Drosophila melanogaster provides a different answer to that found in Manduca. To entice female flies to mate, male flies will sing courtship songs. They do so by vibrating their wings (von Philipsborn, et al., 2011). Using optogenetics our lab has demonstrated that specific activation of the MDH neurons reduces courtship singing behavior.

\section{Conclusions}

Odor plumes are random stochastic stimuli in space and time as air forces move emitted odor molecules. To minimize the uncertainty of stimuli, animals have developed various actions to influence stimulus sampling. Manduca generate a relatively low frequency oscillation in space as 
they cast back and forth through the plume, and a high frequency oscillation in airflow over the antenna caused by successive wing beat cycles. Examination of the casting behavior suggests that these animals are "homing" in on the stimulus; this is common among olfactory search behavior in mammals as well as invertebrates. The reason for the high frequency oscillation is not entirely clear but is consistent with sniffing in that it drives an oscillation in airflow over the olfactory sensory array. Though behavior studies in Manduca examining the effects of pulsed stimuli show a reduction in false positive rates (less unwarranted responses to odorless stimuli) compared to continuous stimuli; this effect mediates the decrease in detection thresholds seen in these studies. This along with increased separation of population odor responses suggests that motor behaviors increase the accuracy of odor processing.

Additionally, Chapters 1 and 2 described a circuit that connects the neural centers governing flight behavior to the olfactory system, whose disruption leads to a decrease in ability for antennal lobe pulse tracking cells to follow the high frequency stimuli. Thus, this circuit facilitates the ability to process its reafferent stimulus possibly by changing the global state of the antennal lobe. Observation of the antennal lobe local field potentials in response to circuit histamine receptor disruption is an increase in $20 \mathrm{~Hz}$ frequency content, this is in contrast to the inconsistent with decrease in pulse tracking among pulse tracking cells decrease. Additionally, histamine application, while increasing pulse tracking, decreases $20 \mathrm{~Hz}$ frequency content in local field activity. One mechanism seen in signal processing theory consistent with this seemingly contradictory finding is stochastic resonance. This is the phenomena where a weak oscillation in increased noise levels facilitates the processing detection of temporally structured subthreshold signals. It does so by periodically increasing the likelihood of a signal detection event spike occurring when close to threshold because the weak signal summates with the oscillation. It is important to note that noise levels were not assessed rather just the $20 \mathrm{~Hz}$ component of antennal lobe activity. Together then, it appears as though the animal's actions lead to an increase in 
accuracy in natural odor processing with an internal signal that modulates the ability of the system to detect an external signal, based on the state of the animal.

The research contained in the previous three chapters explain the anatomical, physiological and evolutionary nature of a novel and unique motor-to-sensory circuit. In particular, we show evidence for how an animal's ecological constraints impart evolutionary changes to nervous system structure which affords optimal stimulus, sensory system, and internal state interactions. The details of these interactions are unknown and represent an interesting experimental model for how nervous systems function. We suggest this research is significant as it may provide an opportunity to understand motor- to- sensory circuits specifically, and more generally, test general theories of how nervous systems function. 


\section{$\underline{\text { References }}$}

\section{Lit Review}

Chemero, A. (2011). Radical embodied cognitive science. MIT press.

Feldman, A. G. (2016). Active sensing without efference copy: referent control of perception. Journal of neurophysiology, 116(3), 960-976.

Fitzpatrick, P., Carello, C., \& Turvey, M. T. (1994). Eigenvalues of the inertia tensor and exteroception by the "muscular sense". Neuroscience, 60(2), 551-568.

Gibson, J. J. (1962). Observations on active touch. Psychological review, 69(6), 477.

Gibson, J. J. (1979). The ecological approach to visual perception: classic edition. Psychology Press.

Gonzalez-Bellido, P. T., Peng, H., Yang, J., Georgopoulos, A. P., \& Olberg, R. M. (2013). Eight pairs of descending visual neurons in the dragonfly give wing motor centers accurate population vector of prey direction. Proceedings of the National Academy of Sciences, 110(2), 696-701.

Holland, N. D. (2003). Early central nervous system evolution: an era of skin brains?. Nature Reviews Neuroscience, 4(8), 617.

Land, M. F., \& Collett, T. S. (1974). Chasing behaviour of houseflies (Fannia canicularis). Journal of comparative physiology, 89(4), 331-357.

McLean, J. H., \& Shipley, M. T. (1987). Serotonergic afferents to the rat olfactory bulb: I. Origins and laminar specificity of serotonergic inputs in the adult rat. Journal of Neuroscience, $7(10), 3016-3028$.

Mischiati, M., Lin, H. T., Herold, P., Imler, E., Olberg, R., \& Leonardo, A. (2015). Internal models direct dragonfly interception steering. Nature, 517(7534), 333.

Olberg, R. M., Worthington, A. H., \& Venator, K. R. (2000). Prey pursuit and interception in dragonflies. Journal of Comparative Physiology A, 186(2), 155-162.

Shaffer, D. M., McBeath, M. K., Roy, W. L., \& Krauchunas, S. M. (2003). A linear optical trajectory informs the fielder where to run to the side to catch fly balls. Journal of Experimental Psychology: Human Perception and Performance, 29(6), 1244.

Verhagen, J. V., Wesson, D. W., Netoff, T. I., White, J. A., \& Wachowiak, M. (2007). Sniffing controls an adaptive filter of sensory input to the olfactory bulb. Nature neuroscience, 10(5), 631.

Wachowiak, M. (2011). All in a sniff: olfaction as a model for active sensing. Neuron, 71(6), 962-973.

Wesson, D. W., Donahou, T. N., Johnson, M. O., \& Wachowiak, M. (2008). Sniffing behavior of mice during performance in odor-guided tasks. Chemical senses, 33(7), 581-596. 


\section{Chapter 1:}

Baker, T. C., Willis, M. A., Haynes, K. F., \& Phelan, P. L. (1985). A pulsed cloud of sex pheromone elicits upwind flight in male moths. Physiological Entomology, 10(3), 257-265.

Beggs, K. T., \& Mercer, A. R. (2009). Dopamine receptor activation by honey bee queen pheromone. Current biology, 19(14), 1206-1209.

Bell, J. S., \& Wilson, R. I. (2016). Behavior reveals selective summation and max pooling among olfactory processing channels. Neuron, 91(2), 425-438.

Burg, M. G., Sarthy, P. V., Koliantz, G., \& Pak, W. L. (1993). Genetic and molecular identification of a Drosophila histidine decarboxylase gene required in photoreceptor transmitter synthesis. The EMBO journal, 12(3), 911-919.

Dacks, A. M., Christensen, T. A., Agricola, H. J., Wollweber, L., \& Hildebrand, J. G. (2005). Octopamineimmunoreactive neurons in the brain and subesophageal ganglion of the hawkmoth Manduca sexta. Journal of Comparative Neurology, 488(3), 255-268.

Dacks, A. M., Reisenman, C. E., Paulk, A. C., \& Nighorn, A. J. (2010). Histamine-immunoreactive local neurons in the antennal lobes of the hymenoptera. Journal of Comparative Neurology, 518(15), 2917-2933.

Dacks, A. M., Riffell, J. A., Martin, J. P., Gage, S. L., \& Nighorn, A. J. (2012). Olfactory modulation by dopamine in the context of aversive learning. Journal of neurophysiology, 108(2), 539-550.

Dai, L. S., Zhu, B. J., Zhao, Y., Zhang, C. F., \& Liu, C. L. (2016). Comparative mitochondrial genome analysis of Eligma narcissus and other Lepidopteran insects reveals conserved mitochondrial genome organization and phylogenetic relationships. Scientific reports, 6, 26387.

Dale, H. H., \& Laidlaw, P. P. (1910). The physiological action of $\beta$-iminazolylethylamine. The Journal of physiology, 41(5), 318-344.

Daly, K. C., \& Smith, B. H. (2000). Associative olfactory learning in the moth Manduca sexta. Journal of Experimental Biology, 203(13), 2025-2038.

Daly, K. C., Durtschi, M. L., \& Smith, B. H. (2001). Olfactory-based discrimination learning in the moth, Manduca sexta. Journal of Insect Physiology, 47(4-5), 375-384.

Haas, H. L., Sergeeva, O. A., \& Selbach, O. (2008). Histamine in the nervous system. Physiological reviews, 88(3), 1183-1241.

Hallem, E. A., Ho, M. G., \& Carlson, J. R. (2004). The molecular basis of odor coding in the Drosophila antenna. Cell, 117(7), 965-979.

Hammer, M., \& Menzel, R. (1995). Learning and memory in the honeybee. Journal of Neuroscience, 15(3), 1617-1630.

Hammer, M., \& Menzel, R. (1998). Multiple sites of associative odor learning as revealed by local brain microinjections of octopamine in honeybees. Learning \& memory, 5(1), 146-156.

Hardie, R. C. (1989). A histamine-activated chloride channel involved in neurotransmission at a photoreceptor synapse. Nature, 339(6227), 704.

Homberg, U. (1994). Distribution of neurotransmitters in the insect brain. Gustav Fischer Verlag. 
Houot, B., Burkland, R., Tripathy, S., \& Daly, K. C. (2014). Antennal lobe representations are optimized when olfactory stimuli are periodically structured to simulate natural wing beat effects. Frontiers in cellular neuroscience, 8, 159.

Ignell, R., Root, C. M., Birse, R. T., Wang, J. W., Nässel, D. R., \& Winther, Å. M. (2009). Presynaptic peptidergic modulation of olfactory receptor neurons in Drosophila. Proceedings of the National Academy of Sciences, 106(31), 13070-13075.

Kaissling, K. E. (2001). Olfactory perireceptor and receptor events in moths: a kinetic model. Chemical Senses, 26(2), 125-150.

Kitching, I. J. (2002). The phylogenetic relationships of Morgan's Sphinx, Xanthopan morganii (Walker), the tribe Acherontiini, and allied long-tongued hawkmoths (Lepidoptera: Sphingidae, Sphinginae). Zoological journal of the Linnean Society, 135(4), 471-527.

Larsson, M. C., Domingos, A. I., Jones, W. D., Chiappe, M. E., Amrein, H., \& Vosshall, L. B. (2004). Or83b encodes a broadly expressed odorant receptor essential for Drosophila olfaction. Neuron, 43(5), 703-714.

Liu, W. W., \& Wilson, R. I. (2013). Glutamate is an inhibitory neurotransmitter in the Drosophila olfactory system. Proceedings of the National Academy of Sciences, 110(25), 10294-10299.

Loudon, C., \& Koehl, M. A. (2000). Sniffing by a silkworm moth: wing fanning enhances air penetration through and pheromone interception by antennae. Journal of experimental Biology, 203(19), 2977-2990.

McClintock, T. S., \& Ache, B. W. (1989). Histamine directly gates a chloride channel in lobster olfactory receptor neurons. Proceedings of the National Academy of Sciences, 86(20), 8137-8141.

Mercer, A. R., Mobbs, P. G., Davenport, A. P., \& Evans, P. D. (1983). Biogenic amines in the brain of the honeybee, Apis mellifera. Cell and tissue research, 234(3), 655-677.

Niven, J. E., Graham, C. M., \& Burrows, M. (2008). Diversity and evolution of the insect ventral nerve cord. Annu. Rev. Entomol., 53, 253-271.

Norlén, P., Ericsson, P., Kitano, M., Ekelund, M., \& Håkanson, R. (2005). The vagus regulates histamine mobilization from rat stomach ECL cells by controlling their sensitivity to gastrin. The Journal of physiology, 564(3), 895-905.

Olsen, S. R., \& Wilson, R. I. (2008). Lateral presynaptic inhibition mediates gain control in an olfactory circuit. Nature, 452(7190), 956.

Perez-Orive, J., Mazor, O., Turner, G. C., Cassenaer, S., Wilson, R. I., \& Laurent, G. (2002). Oscillations and sparsening of odor representations in the mushroom body. Science, 297(5580), 359-365.

Rind, F. C. (1983). The organization of flight motoneurones in the moth, Manduca sexta. Journal of Experimental Biology, 102(1), 239-251.

Sane, S. P., \& Jacobson, N. P. (2006). Induced airflow in flying insects II. Measurement of induced flow. Journal of Experimental Biology, 209(1), 43-56.

Sato, K., Pellegrino, M., Nakagawa, T., Nakagawa, T., Vosshall, L. B., \& Touhara, K. (2008). Insect olfactory receptors are heteromeric ligand-gated ion channels. Nature, 452(7190), 1002.

Stensmyr, M. C., Dweck, H. K., Farhan, A., Ibba, I., Strutz, A., Mukunda, L., ... \& Wicher, D. (2012). A conserved dedicated olfactory circuit for detecting harmful microbes in Drosophila. Cell, 151(6), 1345-1357. 
Tripathy, S., Staudacher, E. M., Peters, O., Kalwar, F., Hatfield, M., \& Daly, K. (2010). Odors pulsed at wing beat frequencies are tracked by primary olfactory networks and enhance odor detection. Frontiers in cellular neuroscience, 4,1 .

Voigt, C. C., \& Winter, Y. (1999). Energetic cost of hovering flight in nectar-feeding bats (Phyllostomidae: Glossophaginae) and its scaling in moths, birds and bats. Journal of Comparative Physiology B, 169(1), 38-48.

Vosshall, L. B., Wong, A. M., \& Axel, R. (2000). An olfactory sensory map in the fly brain. Cell, 102(2), 147159.

Wicher, D., Schäfer, R., Bauernfeind, R., Stensmyr, M. C., Heller, R., Heinemann, S. H., \& Hansson, B. S. (2008). Drosophila odorant receptors are both ligand-gated and cyclic-nucleotide-activated cation channels. Nature, 452(7190), 1007.

Willis, M. A., \& Baker, T. C. (1994). Behaviour of flying oriental fruit moth males during approach to sex pheromone sources. Physiological Entomology, 19(1), 61-69.

Willis, M. A., \& Arbas, E. A. (1991). Odor-modulated upwind flight of the sphinx moth, Manduca sexta L. Journal of Comparative Physiology A, 169(4), 427-440.

Willmott, A. P., \& Ellington, C. P. (1997). The mechanics of flight in the hawkmoth Manduca sexta. I. Kinematics of hovering and forward flight. Journal of experimental Biology, 200(21), 2705-2722.

Wong, A. M., Wang, J. W., \& Axel, R. (2002). Spatial representation of the glomerular map in the Drosophila protocerebrum. Cell, 109(2), 229-241.

Zheng, Y., Hirschberg, B., Yuan, J., Wang, A. P., Hunt, D. C., Ludmerer, S. W., ... \& Cully, D. F. (2002). Identification of two novel Drosophila melanogaster histamine-gated chloride channel subunits expressed in the eye. Journal of Biological Chemistry, 277(3), 2000-2005.

Chapter 2:

Alexander, R. D. (1962). Evolutionary change in cricket acoustical communication. Evolution, 16(4), 443467.

Azim, E., Jiang, J., Alstermark, B., \& Jessell, T. M. (2014). Skilled reaching relies on a V2a propriospinal internal copy circuit. Nature, 508(7496), 357.

Bell, C. C. (1981). An efference copy which is modified by reafferent input. Science, 214(4519), 450-453.

Bender, J. A., \& Dickinson, M. H. (2006). Visual stimulation of saccades in magnetically tethered Drosophila. Journal of Experimental Biology, 209(16), 3170-3182.

Brainard, M. S., \& Doupe, A. J. (2000). Auditory feedback in learning and maintenance of vocal behaviour. Nature Reviews Neuroscience, 1(1), 31.

Chalfie, M., Sulston, J. E., White, J. G., Southgate, E., Thomson, J. N., \& Brenner, S. (1985). The neural circuit for touch sensitivity in Caenorhabditis elegans. Journal of Neuroscience, 5(4), 956-964.

Colby, C. L., \& Goldberg, M. E. (1992). The updating of the representation of visual space in parietal cortex by intended eye movements. Science, 255(5040), 90-92. 
Collett, T. S., \& Land, M. F. (1975). Visual control of flight behaviour in the hoverflySyritta pipiens L. Journal of comparative physiology, 99(1), 1-66.

Crapse, T. B., \& Sommer, M. A. (2008). Corollary discharge across the animal kingdom. Nature Reviews Neuroscience, 9(8), 587.

Elliott, C. J. H. (1983). Wing hair plates in crickets: physiological characteristics and connections with stridulatory motor neurones. Journal of experimental biology, 107(1), 21-47.

Griisser, O. J. (1995). On the history of the ideas of efference copy and reafference. In Essays in the History of the Physiological Sciences: Proceedings of a Network Symposium of the European Association for the History of Medicine and Health Held at the University Louis Pasteur, Strasbourg, on March (Vol. 26, p. 35).

Hedwig, B. (2000). Control of cricket stridulation by a command neuron: efficacy depends on the behavioral state. Journal of Neurophysiology, 83(2), 712-722.

Herculano-Houzel, S. (2010). Coordinated scaling of cortical and cerebellar numbers of neurons. Frontiers in neuroanatomy, 4, 12.

Houk, J. C., Buckingham, J. T., \& Barto, A. G. (1996). Models of the cerebellum and motor learning. Behavioral and brain sciences, 19(3), 368-383.

Jordan, K., Calderone, D., Rubin, A., \& Wickenden, A. E. (2010). A review of biological communication mechanisms applicable to small autonomous systems (No. ARL-TR-5340). ARMY RESEARCH LAB ADELPHI MD.

Kim, A. J., Fenk, L. M., Lyu, C., \& Maimon, G. (2017). Quantitative predictions orchestrate visual signaling in Drosophila. Cell, 168(1-2), 280-294.

Kim, A. J., Fitzgerald, J. K., \& Maimon, G. (2015). Cellular evidence for efference copy in Drosophila visuomotor processing. Nature neuroscience, 18(9), 1247.

Mischiati, M., Lin, H. T., Herold, P., Imler, E., Olberg, R., \& Leonardo, A. (2015). Internal models direct dragonfly interception steering. Nature, 517(7534), 333.

Mohr, C., Roberts, P. D., \& Bell, C. C. (2003). The mormyromast region of the mormyrid electrosensory lobe. I. Responses to corollary discharge and electrosensory stimuli. Journal of Neurophysiology, 90(2), 1193-1210.

Ouellette, M. H., Desrochers, M. J., Gheta, I., Ramos, R., \& Hendricks, M. (2018). A Gate-and-Switch Model for Head Orientation Behaviors in Caenorhabditis elegans. eNeuro, 5(6).

Poulet, J. F., \& Hedwig, B. (2002). A corollary discharge maintains auditory sensitivity during sound production. Nature, 418(6900), 872.

Poulet, J. F., \& Hedwig, B. (2003). Corollary discharge inhibition of ascending auditory neurons in the stridulating cricket. Journal of Neuroscience, 23(11), 4717-4725.

Poulet, J. F., \& Hedwig, B. (2006). The cellular basis of a corollary discharge. Science, 311(5760), 518522.

Schnell, Á., Joesch, M., Forstner, F., Raghu, S. V., Otsuna, H., Ito, K., ... \& Reiff, D. F. (2010). Processing of horizontal optic flow in three visual interneurons of the Drosophila brain. Journal of neurophysiology, 103(3), 1646-1657.

Scott, E. K., Raabe, T., \& Luo, L. (2002). Structure of the vertical and horizontal system neurons of the lobula plate in Drosophila. Journal of Comparative Neurology, 454(4), 470-481. 
Sommer, M. A., \& Wurtz, R. H. (2002). A pathway in primate brain for internal monitoring of movements. Science, 296(5572), 1480-1482.

Sommer, M. A., \& Wurtz, R. H. (2006). Influence of the thalamus on spatial visual processing in frontal cortex. Nature, 444(7117), 374.

Umeno, M. M., \& Goldberg, M. E. (1997). Spatial processing in the monkey frontal eye field. I. Predictive visual responses. Journal of neurophysiology, 78(3), 1373-1383.

Troyer, T. W., \& Doupe, A. J. (2000). An associational model of birdsong sensorimotor learning I. Efference copy and the learning of song syllables. Journal of Neurophysiology, 84(3), 1204-1223.

von Holst, E., \& Mittelstaedt, H. (1971). The principle of reafference: Interactions between the central nervous system and the peripheral organs. Perceptual processing: Stimulus equivalence and pattern recognition, 41-72.

White, J. G., Southgate, E., Thomson, J. N., \& Brenner, S. (1986). The structure of the nervous system of the nematode Caenorhabditis elegans. Philos Trans R Soc Lond B Biol Sci, 314(1165), 1-340.

Zaretsky, M., \& Rowell, C. F. (1979). Saccadic suppression by corollary discharge in the locust. Nature, 280(5723), 583.

\section{Chapter 3:}

Corcoran, J. A., Sonntag, Y., Andersson, M. N., Johanson, U., \& Löfstedt, C. (2018). Endogenous insensitivity to the Orco agonist VUAA1 reveals novel olfactory receptor complex properties in the specialist fly Mayetiola destructor. Scientific reports, 8(1), 3489.

Dekker, T., Ibba, I., Siju, K. P., Stensmyr, M. C., \& Hansson, B. S. (2006). Olfactory shifts parallel superspecialism for toxic fruit in Drosophila melanogaster sibling, D. sechellia. Current Biology, 16(1), 101-109.

Krieger, J., Klink, O., Mohl, C., Raming, K., \& Breer, H. (2003). A candidate olfactory receptor subtype highly conserved across different insect orders. Journal of Comparative Physiology A, 189(7), 519-526.

Kritsky, G. (1991). Darwin's Madagascan hawk moth prediction. American Entomologist, 37(4), 206-210.

Larsson, M. C., Domingos, A. I., Jones, W. D., Chiappe, M. E., Amrein, H., \& Vosshall, L. B. (2004). Or83b encodes a broadly expressed odorant receptor essential for Drosophila olfaction. Neuron, 43(5), 703-714.

Lavista-Llanos, S., Svatoš, A., Kai, M., Riemensperger, T., Birman, S., Stensmyr, M. C., \& Hansson, B. S. (2014). Dopamine drives Drosophila sechellia adaptation to its toxic host. Elife, 3, e03785.

Lizbinski, K. M., Metheny, J. D., Bradley, S. P., Kesari, A., \& Dacks, A. M. (2016). The anatomical basis for modulatory convergence in the antennal lobe of Manduca sexta. Journal of Comparative Neurology, 524(9), 1859-1875. 
Martin, J. P., Beyerlein, A., Dacks, A. M., Reisenman, C. E., Riffell, J. A., Lei, H., \& Hildebrand, J. G. (2011). The neurobiology of insect olfaction: sensory processing in a comparative context. Progress in neurobiology, 95(3), 427-447.

Obara, Y. (1979). Bombyx mori mating dance: An essential in locating the female. Applied Entomology and Zoology, 14(1), 130-132.

Piersanti, S., Frati, F., Conti, E., Rebora, M., \& Salerno, G. (2014). The sense of smell in Odonata: an electrophysiological screening. Journal of insect physiology, 70, 49-58.

Price, P. W. (1997). Insect ecology. John Wiley \& Sons.

Robertson, H. M., Warr, C. G., \& Carlson, J. R. (2003). Molecular evolution of the insect chemoreceptor gene superfamily in Drosophila melanogaster. Proceedings of the National Academy of Sciences, 100(suppl 2), 14537-14542.

Rössler, W., \& Brill, M. F. (2013). Parallel processing in the honeybee olfactory pathway: structure, function, and evolution. Journal of Comparative Physiology A, 199(11), 981-996.

Stensmyr, M. C., Dweck, H. K., Farhan, A., Ibba, I., Strutz, A., Mukunda, L., ... \& Wicher, D. (2012). A conserved dedicated olfactory circuit for detecting harmful microbes in Drosophila. Cell, 151(6), 13451357.

Sweeney, A., Jiggins, C., \& Johnsen, S. (2003). Insect communication: polarized light as a butterfly mating signal. Nature, 423(6935), 31.

Wada-Katsumata, A., Silverman, J., \& Schal, C. (2013). Changes in taste neurons support the emergence of an adaptive behavior in cockroaches. Science, 340(6135), 972-975.

Willis, M. A., \& Baker, T. C. (1994). Behaviour of flying oriental fruit moth males during approach to sex pheromone sources. Physiological Entomology, 19(1), 61-69.

\section{Conclusions:}

Akashi, H. D., Chen, P. J., Akiyama, T., Terai, Y., Wakakuwa, M., Takayama, Y., ... \& Arikawa, K. (2018). Physiological responses of ionotropic histamine receptors, PxHCLA and PxHCLB, to neurotransmitter candidates in a butterfly, Papilio xuthus. Journal of Experimental Biology, 221(21), jeb183129.

Alejevski, F., Saint-Charles, A., Michard-Vanhée, C., Martin, B., Galant, S., Vasiliauskas, D., \& Rouyer, F. (2019). The HisCl1 histamine receptor acts in photoreceptors to synchronize Drosophila behavioral rhythms with light-dark cycles. Nature communications, 10(1), 252.

Crapse, T. B., \& Sommer, M. A. (2008). Corollary discharge across the animal kingdom. Nature Reviews Neuroscience, 9(8), 587. 
Elias, M. S., \& Evans, P. D. (1983). Histamine in the insect nervous system: distribution, synthesis and metabolism. Journal of neurochemistry, 41(2), 562-568.

Gisselmann, G., Pusch, H., Hovemann, B. T., \& Hatt, H. (2002). Two cDNAs coding for histamine-gated ion channels in D. melanogaster. Nature neuroscience, 5(1), 11.

Hardie, R. C. (1989). A histamine-activated chloride channel involved in neurotransmission at a photoreceptor synapse. Nature, 339(6227), 704.

Kupenova, P., \& Yusein-Myashkova, S. (2012). Participation of the histamine receptor encoded by the gene hclB (HCLB) in visual sensitivity control: an electroretinographic study in Drosophila melanogaster. Molecular vision, 18, 2497.

McClintock, T. S., \& Ache, B. W. (1989). Histamine directly gates a chloride channel in lobster olfactory receptor neurons. Proceedings of the National Academy of Sciences, 86(20), 8137-8141.

Mischiati, M., Lin, H. T., Herold, P., Imler, E., Olberg, R., \& Leonardo, A. (2015). Internal models direct dragonfly interception steering. Nature, 517(7534), 333.

Pantazis, A., Segaran, A., Liu, C. H., Nikolaev, A., Rister, J., Thum, A. S., ... \& Hardie, R. C. (2008). Distinct roles for two histamine receptors (hclA and $\mathrm{hclB}$ ) at the Drosophila photoreceptor synapse. Journal of Neuroscience, 28(29), 7250-7259.

Perez-Orive, J., Mazor, O., Turner, G. C., Cassenaer, S., Wilson, R. I., \& Laurent, G. (2002). Oscillations and sparsening of odor representations in the mushroom body. Science, 297(5580), 359-365.

Schnaitmann, C., Haikala, V., Abraham, E., Oberhauser, V., Thestrup, T., Griesbeck, O., \& Reiff, D. F. (2018). Color processing in the early visual system of Drosophila. Cell, 172(1-2), 318-330.

von Philipsborn, A. C., Liu, T., Jai, Y. Y., Masser, C., Bidaye, S. S., \& Dickson, B. J. (2011). Neuronal control of Drosophila courtship song. Neuron, 69(3), 509-522.

Witte, I., Kreienkamp, H. J., Gewecke, M., \& Roeder, T. (2002). Putative histamine-gated chloride channel subunits of the insect visual system and thoracic ganglion. Journal of neurochemistry, 83(3), 504-514.

Yusein, S., Velikova, N., Kupenova, P., Hardie, R., Wolstenholme, A., \& Semenov, E. (2008). Altered ivermectin pharmacology and defective visual system in Drosophila mutants for histamine receptor HCLB. Invertebrate neuroscience, 8(4), 211-222.

Yusein, S., Wolstenholme, A., \& Semenov, E. (2010). Functional consequences of mutations in the Drosophila histamine receptor HCLB. Journal of insect physiology, 56(1), 21-27.

Zheng, Y., Hirschberg, B., Yuan, J., Wang, A. P., Hunt, D. C., Ludmerer, S. W., ... \& Cully, D. F. (2002). Identification of two novel Drosophila melanogaster histamine-gated chloride channel subunits expressed in the eye. Journal of Biological Chemistry, 277(3), 2000-2005. 


\section{Acknowledgements}

This work would not have been possible without the help and support from my advisor Kevin C. Daly and the rest of my committee members. My colleagues Phillip C. Chapman, Rex Burkland, Benjamin Houot and Erich Staudacher along with undergraduates and fellow graduate students in the biology department. Particularly, those in the neurobiology groups in Andrew Dacks, Gary Marsat and Sarah Farris's labs. Finally, I am grateful for my family and their support throughout the years. 
Appendix A:

\title{
A flight sensory-motor to olfactory processing circuit in the moth Manduca sexta
}

\author{
Samual P. Bradley*, Phillip D. Chapman, Kristyn M. Lizbinski, Kevin C. Daly", Andrew \\ M. Dacks ${ }^{\#}$
}

West Virginia University, Department of Biology, Morgantown, West Virginia United States of America

\# Denotes co-senior authorship

* Correspondence: Samual P. Bradley, West Virginia Universtiy, Department of Biology, 53 Campus Drive Morgantown, West Virginia, 26501, United States of America

sbradle7@mix.wvu.edu

Keywords: modulation, histamine, olfaction, insect, flight.

List of Abbreviations

$\begin{array}{ll}\text { AL } & \text { Antennal lobe } \\ \text { AMMC } & \text { Antennal mechanosensory and motor center } \\ \text { ATR } & \text { allatotropin } \\ \text { BRP } & \text { bruchpilot } \\ \text { BSA } & \text { Bovine serum albumin } \\ \text { FMRF } & \text { FMRF-amide } \\ \text { HA } & \text { Histamine } \\ \text { HisClA } & \text { Histamine A receptor } \\ \text { LAC } & \text { Larval antennal center } \\ \text { LNs } & \text { Local interneurons } \\ \text { MDHn } & \text { Mesothoracic deutocerebrum histamine neurons } \\ \text { MsHisClB } & \text { Manduca sexta histamine B receptor } \\ \text { MsG } & \text { Mesothoracic ganglia } \\ \text { ORNs } & \text { Olfactory receptor neurons } \\ \text { PNs } & \text { Projection neurons } \\ \text { SEZ } & \text { Sub-esophageal zone }\end{array}$

Acknowledgements: We would like to thank Sarah Farris for technical advice and for editing the manuscript, Jackie Metheny for performing the Western Blot for the MsHisClB antibody. This work was supported by RO3 DC13997-01 to AMD, and RO1 DC009417 to KCD. 


\begin{abstract}
Neural circuits projecting information from motor to sensory pathways are common across sensory domains. These circuits typically modify sensory function as a result of motor pattern activation; this is particularly so in cases where the resultant behavior affects the sensory experience or its processing. However, such circuits have not been observed projecting to an olfactory pathway in any species despite well characterized active sampling behaviors that produce reafferent mechanical stimuli, such as sniffing in mammals and wing beating in the moth Manduca sexta. In this study we characterize a circuit that connects a flight sensory-motor center to an olfactory center in Manduca. This circuit consists of a single pair of histamine immunoreactive (HA-ir) neurons that project from the mesothoracic ganglion to innervate a subset of ventral antennal lobe (AL) glomeruli. Furthermore, within the AL we show that the Manduca sexta histamine B receptor (MsHisClB) is exclusively expressed by a subset of GABAergic and peptidergic LNs, which broadly project to all olfactory glomeruli. Finally, the HA-ir cell pair is present in fifth stage instar larvae; however, the absence of MsHisClB-ir in the larval antennal center (LAC) indicates that the circuit is incomplete prior to metamorphosis and importantly prior to the expression of flight behavior. Although the functional consequences of this circuit remain unknown, these results provide the first detailed description of a circuit that interconnects an olfactory system with motor centers driving flight behaviors including odor-guided flight.
\end{abstract}

\title{
Introduction
}

Animals exhibit stereotypical search behaviors in pursuit of potential food sources or mating partners. More specifically, some animals employ sampling strategies where rhythmic motor patterns optimize the interaction between stimuli and their affected sensory systems. Consequently, many of these motor systems project to and modulate how sensory systems process this information. For example, saccadic eye movements allow us to focus on objects despite having a fast adapting visual system (Martinez-Conde et al., 2006). Here the neural circuits driving these small movements also send a signal canceling the perception of a moving scene, therefore affording proper behavioral responses to other stimuli in the environment (Zaretsky and Rowell, 1979; Ross et al., 2001). Other motor to sensory circuits have been shown to amplify self-induced communication signals (Mohr et al., 2003), inhibit reflex responses (Chalfie et al., 1985) and are involved in sensory/motor planning (Sommer and Wurtz, 2002; Brainard and Doupe, 2000). While work in other sensory systems have made significant progress in characterizing motor to sensory circuits (Crapse and Sommer, 2014), it is not clear whether such circuits are present in the olfactory system.

When tracking odors, animals typically exhibit behaviors, such as sniffing, that periodically structure olfactory stimuli (Halpern, 1983). Each sniff cycle draws odor-laden air into the nasal cavity during inhalation and forces air out during exhalation, thus imposing a temporal structure on air/olfactory receptor interactions that persists in the absence of odor (Adrian, 1942; Kepecs et al., 2007). In this manner, sniffing couples reafferent mechanical stimuli with odor stimuli resulting in a temporally structured stimulus that improves physiological (Verhagen et al., 2007), and presumably behavioral performance. In the moth Manduca sexta, wing beating causes high frequency oscillations in airflow over the antennae in a manner analogous to sniffing (Sane and Jacobson, 2006). These periodic signals have a potentially strong effect on odor-receptor interactions in moths (Loudon et al., 1994; Loudon and Koehl, 2000) and are effectively tracked 
by antennal and antennal lobe (AL) neurons (Tripathy et al., 2010). This implies that at least part of the temporal structure of encoding neuron activity is driven by time-dependent fluctuations in stimulus concentration (Christensen et al., 1998; Daly et al., 2011), driven by wing-beating. Simulating wing-beating effects on odor exposure by pulsing odor stimuli at wing beat frequencies increases separation of neural ensemble representations for different odors (Houot et al., 2014) and enhances behavioral performance in psychophysical assays of olfactory acuity (Tripathy et al., 2010; Daly et al., 2013). While AL neurons can track pulsed stimuli when the neck connective is intact (Houot et al., 2014), AL neurons are unable to do so when using isolated head preparations (Tripathy et al., 2010; Christensen et al., 1998). This suggests that the AL receives input from flight sensorimotor centers that affects the temporal fidelity with which the AL encodes odors (Christensen et al., 1998; Tripathy et al., 2010). However, relatively little is known about neural circuits connecting flight sensory-motor centers and the AL.

There is limited data describing input from flight sensory-motor centers to the ALs of Manduca. This circuit consists of a single pair of histamine (HA) immunoreactive neurons that project from the mesothoracic ganglion (MsG) and bilaterally innervate both ALs and the antennal mechanosensory and motor center (AMMC; Homberg, 1994; Homberg and Hildebrand, 1991). The purpose of this study was to provide a detailed morphological description of these mesothoracic to deutocerebral histaminergic neurons (MDHn) and to identify candidate post synaptic targets. Using immunohistochemistry, we found that the MDHns ramify in a subset of ventral glomeruli in the AL, the AL isthmus, and the coarse neuropil. A subset of GABAergic LNs along with one FMRFamide-ir and one allatotropin-ir (ATR-ir) local interneuron express the Manduca homologue of the histamine B receptor subtype (MsHisClB) and thus represent candidate postsynaptic targets of the MDHns. Furthermore, although the MDHns are present in larvae and survive metamorphosis there is no expression of the MsHisClB receptor in larval antennal center (LAC) neurons until after pupation has occurred, suggesting the MDHns only affect olfactory processing in adults. The MDHns therefore represent a novel circuit that provides a potential source of information from a flight sensory-motor integration system to the olfactory system.

\section{Materials and Methods}

\section{Animals}

Animals were raised using a standard diet (Bell and Joachim, 1976) and rearing procedures (Tripathy et al., 2010). Adult moths were kept in brown paper bags and placed in an incubator (Percival Scientific Inc.; 166VLC8) where they were exposed to a 16/8 reverse light dark cycle set to $25^{\circ} \mathrm{C}$ and $75 \%$ humidity. Approximately 10 male or female moths aged 3-9 days were used for all experimental groups. For larval studies, stage 5 instar larvae were dissected with trachea removed. Ten larval nervous systems were used for developmental experiments.

\section{Immunohistochemistry}

Immunolabeling was performed as described previously (Dacks et al., 2010) on both sectioned and whole-mount brains depending upon the preparation. For HA immunolabeling, brains were placed in a $4 \% \mathrm{~N}$-3-dimethylaminopropyl-N'-ethylcarbodiimide (Sigma Aldrich, 03449) pre-fixative for 3-4 hours at $4^{\circ} \mathrm{C}$, before being fixed overnight in $4 \%$ paraformaldehyde 
(Electron Microscope Sciences, 15710) in 1\% phosphate buffered saline (PBS; Sigma Aldrich, SLBC5890) at $4^{\circ} \mathrm{C}$. For the MsHisClB antibody, brains were placed in $4 \%$ paraformaldehyde (Electron Microscopy Sciences, 15710; $\mathrm{pH} 7.3-7.5$ ) at $4^{\circ} \mathrm{C}$ overnight. Following fixation, brains were washed in PBS (pH 6.9). For sectioned tissue, adult brains and ganglia were embedded in 5\% agarose (Sigma Aldrich, SLBJ3744V) and sectioned between 50 and $250 \mu \mathrm{m}$ (depending on the antibody) using a Leica VT 1000 S vibrating microtome. The tissue was washed in PBS with $0.5 \%$ Triton $^{\mathrm{TM}}-\mathrm{X} 100$ (PBST; Sigma Aldrich, 110M0009V), blocked for 1 hour with 2\% IgG-free bovine serum albumin (BSA; Jackson Laboratory, 001-000-162) and incubated in primary antibody in blocking solution with 5mM with sodium azide (PBSAT; Fisher Scientific, S2271). Brains were washed and blocked as above, then incubated in secondary antibody (1:1000 Alexa 488, 546, or 633 in PBSAT; Alexa fluor ${ }^{\circledR}$; Lifescience Technologies) overnight at room temperature except for experiments using MsHisClB and/or GABA in which tissue was incubated at $4^{\circ} \mathrm{C}$. SYTO 59 (a nuclear label; Invitrogen ${ }^{\mathrm{TM}}$; S11341) was used to outline the LAC. Tissue was washed several times in Tris Buffered Saline (TBS; Bio-Rad, 170-6435) and the tissue was incubated in 1:10,000 SYTO 59 in Tris-HCl (Fisher Scientific, BP153 for 60 minutes before mounting. All tissue was washed in PBST and PBS, then run through an ascending glycerol (Sigma Aldrich, BCBN3647V) series $\left(40 \%, 60 \%\right.$ and $80 \%$ ) and mounted in Vectashield ${ }^{\circledR}$ (Vector laboratories, ZA1222). For whole-mount preparations, tissue was run through an ascending ethanol (Sigma Aldrich, SHBF6704V) dilution series (30\%, 50\%, 70\%, 95\%, and 100\%) for 10 minutes each (after the PBS wash), a 1:1 ethanol methyl salicylate solution for 15 minutes, and finally mounted in 100\% methyl salicylate (Fisher Scientific, MFCD00002214). All primary antibody information (including dilutions used, manufacturer, host-species, immunogen and RRID) is included in Table 1.

\section{Antibody Manufacturing and Characterization}

\section{Rabbit anti-histamine}

The HA antiserum was raised against synthetic HA conjugated via a carbodiimide linker to succinylated keyhole limpet hemocyanin. Control studies showed that the antibody had no cross reactivity with L-histidine or L-histidine containing peptides, and pre-adsorbing the antiserum with the HA conjugate eliminates labeling (Immunostar histochemical histamine antiserum specification sheet) as did an RNAi knock down of histidine decarboxylase in Drosophila (Melzig et al., 1996). Finally, pre-adsorbing the HA antiserum against keyhole limpet hemocyanin alone did not eliminate HA labeling in Bombus impatiens (Dacks et al., 2010). Pre-adsorption controls in Manduca tissue were performed by incubating the rabbit anti-HA antiserum for $24 \mathrm{hrs}$ in blocking solution (1mg/ml BSA in PBSAT) with HA (Sigma Aldrich, H7250) at a ratio of 10:1 HA:antiserum. Non-pre-adsorbed controls in which rabbit anti-HA antibody was incubated in parallel under identical conditions resulted in immunolabeling (Fig. 1A; n=5) whereas preadsorbing the antibody abolished all staining in Manduca optic lobe tissue (Fig. 1B; n=5).

\section{Mouse anti-bruchpilot}

Bruchpilot (Brp) is homologous to the protein ELKS/CAST in mammals and functions as a structural protein at presynaptic active zones (Wagh et al., 2006). The Brp antiserum was raised against Brp and western blots showed two bands for two isoforms of the Brp protein in Drosophila (Wagh et al., 2006). Brp labeling was absent in Brp mutants (Kittel et al., 2006) and has been 
shown to bind to amino acid sequence 1390-1740 (Fouquet et al., 2009). The Brp antiserum produced a single band at the predicted weight for the Manduca homologue of Brp in western blots using Manduca brain tissue (Lizbinski, et al., In Press). The purpose of using the anti-Brp antibody in this study was to highlight the boundaries of neuropil, rather than to make any conclusions about the distribution of the Manduca homolog of Brp.

\section{Mouse anti-GABA}

GABA antiserum was raised against GABA coupled to BSA with glutaraldehyde. Controls show that the antibody was highly specific to GABA and did not react with other amino acid BSA conjugates (Abcam data sheet). Pre-adsorption controls were performed by incubating the mouse anti-GABA antiserum for $24 \mathrm{hrs}$ in blocking solution $(1 \mathrm{mg} / \mathrm{ml}$ BSA in PBSAT) with GABA (Sigma Aldrich, cat \# A2129) at a ratio of 10:1 GABA:antiserum. Non-pre-adsorbed controls in which mouse anti-GABA antibody was incubated in parallel under identical conditions resulted in strong immunolabeling (Fig. $1 \mathrm{C} ; \mathrm{n}=5$ ) whereas preadsorbing the antibody abolished all staining in Manduca AL tissue (Fig. 1D; $\mathrm{n}=5$ ).

\section{Rabbit anti-FMRFamide}

FMRFamide antiserum was provided by Dr. Eve Marder and was raised against synthetic RF-amide coupled to bovine thyroglobulin with glutaraldehyde (Marder et al., 1987). Preadsorbing the antiserum against synthetic FMRFamide eliminated labeling in larval Manduca nervous tissue (Witten and Truman, 1996).

\section{Rabbit anti-allatotropin}

Allatotropin (ATR) antiserum was provided by Dr. Jan Veenstra and raised against purified ATR coupled to thyroglobulin using glutaraldehyde (Veenstra and Hagedorn, 1993). ELISA did not show cross reactivity with 100 pmol corazonin, vasopressin, leucokinin IV, or proctolin, but did show significant immunoreacitivity to the truncated 6-13 analogue of Manduca ATR (Veenstra and Hagedorn, 1993). Preadsorbing the antiserum against ATR eliminated immunolabeling in Manduca tissue (Utz et al., 2007).

\section{Rabbit anti-MsHisClB}

To determine the amino acid sequence of the Manduca homologue of the HA B-type receptor (MsHisClB), we used the Manduca genome (Agricultural Pest Genomics Resource Database: (www.agripestbase.org) to perform a forward protein BLAST analysis of the Drosophila melanogaster histamine B-type receptor (HisClB) amino acid sequence (ACA13298.1). The top match from the Manduca genome had an e-value of 0.0 (Msex2.04603RA). We then reverse blasted this sequence from the Manduca genome into the Drosophila genome in NCBI and the first 3 matches were Drosophila HisClB isoforms (NP_650116.2, NP_731632.1 and NP_001163591.1), all of which had e-values of 0.0. The next highest match from the Drosophila genome was the HisClA receptor (otherwise known as "ora transientless"; NP_524406.1) which is the other of the two histamine receptor types in Drosophila (Zheng et al. 2002) and had e-values of 3e-148 which is consistent with both histamine receptor types having high sequence homology (Zheng et al. 2002; Jones et al 2010). To ensure that there were not two predicted amino acid sequences from the Manduca genome with high sequence homology to the 
Drosophila HisClB receptor, we took the amino acid sequence from the Manduca genome with the second highest e-value for the Drosophila MsHisCIB (Msex2.04216-RA; e-value = 1e-119) and ran a BLAST analysis of this sequence in the Drosophila genome. The BLAST analysis resulted in an e-value of 7.37e-158 for the Drosophila ora transientless indicating that the Manduca protein with the next closest sequence similarity to Drosophila HisClA was likely not the MsHisClB homologue. Figure $1 \mathrm{E}$ is a sequence alignment of the Manduca HisClB receptor (MsHisClB) with the sequences for known histamine B receptors from Drosophila melanogaster (ACA13298.1), Apis meliferia (ABG75740.1), and Nasonia vitripennis (ACZ51422.1) (Jones et al., 2010) using the EMBL-EBI Clustal omega tool (Sievers et al., 2011; http://www.ebi.ac.uk/Tools/msa/clustalo/).

Custom affinity purified antibodies were generated in rabbit (Bethyl laboratories) using Cys-VNPDIELPQLD as the immunogenic sequence. The immunogenic sequence was highly conserved across D. melanogaster, A. mellifera and N. vitripennis (Fig. 1E). For western blots, adult brains were placed in BoltTM LDS Sample Buffer (Life Technologies, B0007, Life Technologies) with protease inhibitor cocktail (Research Products International, P50900) and DNase I (Invitrogen, 18068-015) and kept on ice for homogenization with a pestle. Samples were heated in a water bath for 10 minutes at $95^{\circ} \mathrm{C}$. We used the Novex ${ }^{\circledR}$ Bolt ${ }^{\mathrm{TM}}$ Gel Electrophoresis System (Life Technologies) with Tris-Glycine SDS Running Buffer at 165V for 2.5 hours and Bolt $^{\mathrm{TM}} 4-12 \%$ Bis-Tris Plus Precast Gels (BG04120BOX) to resolve proteins. We used the iBlot@ Gel Transfer Device (Life Technologies, IB 1001) program P0 (20 V for $1 \mathrm{~min}, 23 \mathrm{~V}$ for 4 min, 25 $\mathrm{V}$ for $2 \mathrm{~min}$ ) to transfer proteins to nitrocellulose membranes (nitrocellulose iBlot ${ }^{\circledR}$ Transfer Stacks, Life Technologies, IB3010-01). The WesternBreeze ${ }^{\circledR}$ Chromogenic Western Blot Immunodetection Kit (WB7105, anti-rabbit) protocol was used to detect proteins. Images of membranes were taken with FluorChem Q using Alpha View Analysis Software. The amino acid sequence of the MsHisClB receptor has a predicted molecular weight of 36kDa (ExPASy Bioinformatics Resource Portal: http://web.expasy.org/compute_pi/) and the western blot resulted in a single band at the predicted molecular weight of $36 \mathrm{kDa}$ (Fig. 1F). Histamine is the primary neurotransmitter of arthropod photoreceptors (Hardie, 1989; Stuart, 1999) and the HisClB receptor is expressed by glial cells in the lamina of Drosophila (Pantazis et al., 2008). Consistent with this, we observed a band of MsHisClB labeling in the lamina (Fig. 1G). Pre-adsorbing the MsHisClB antibody in a 10:1 antigenic peptide to antibody solution eliminated all labeling (Figure 1H). Preadsorption controls were run concurrently with samples incubated in antibody that had not been pre-absorbed with the antigenic peptide (Fig. 1G), but otherwise treated identically. Scan settings were increased slightly for preadsorbed tissue so that autoflourescence outlined brain structures. Finally, RT-PCR of the insect histamine A receptor showed no band at the predicted height for the receptor suggesting that the $\mathrm{MsHisClB}$ receptor is the only $\mathrm{HA}$ receptor expressed in $\mathrm{AL}$ tissue.

\section{Direct fluorescent tagging of primary antibodies}

Both neuropeptide antibodies (anti-FMRFamide and anti-ATR) and the MsHisClB receptor antibody were produced in rabbit hosts. Therefore, to double label using the neuropeptides and the rabbit anti-MsHisClB antibodies we directly fluorescently tagged each primary antibody using the APEX antibody labeling kit (Life Technologies, A10468 488, A10475 for 647; Woo et al., 2010). This method covalently bonds the IgG antibody to a fluorescent label, and therefore eliminates cross reactivity of secondary antibodies with primary antibodies raised in the same animal. To remove contaminants, the labeling tip was hydrated with $100 \mathrm{uL}$ of wash buffer to 
which 10-20 ug of IgG antibody is added and eluted with a syringe: $10 \mathrm{uL}$ of MsHisClB, and $1 \mathrm{uL}$ of both FMRFamide and ATR antibody respectively. This solution was then combined with reactive dye (either Alexa 488 or Alexa 647) containing $2 \mathrm{uL}$ of DMSO and $18 \mathrm{uL}$ of labeling buffer. This solution then incubated for 2 hours at room temperature. The solution was washed with $50 \mathrm{uL}$ of buffer and eluted through the tip. Finally, $40 \mathrm{uL}$ of elution buffer is eluted through the tip and mixed with $10 \mathrm{uL}$ of neutralization buffer to yield a final volume of $\sim 50 \mathrm{uL}$ of solution. This solution was then diluted in $350 \mathrm{uL}$ of PBSAT and tissue was incubated for 48 hours at $4^{\circ} \mathrm{C}$.

\section{Retrograde dye fills of AL PN output tracks}

Two to three day old moths were restrained with dental wax and the head capsule was opened. Once opened, dextran-Texas Red dye (ThermoFisher, D-1863) was injected into either the mushroom bodies or lateral horn (the two projection fields of AL PNs). Animals were kept alive for 2-3 days post injection and were fed sugar water to ensure that they survived. After 2-3 days, animals were sacrificed and ran through the HA staining protocol described above.

\section{Ablation Studies}

To definitively demonstrate that the MDHns are the sole source of HA to the AL, lesion experiments were performed to ablate ascending HA-ir fibers from the MDHns or more posterior HA-ir neurons in the metathoracic and abdominal ganglia. At 1-3 days post-eclosion the connective between the sub-esophageal zone (SEZ) and the prothoracic ganglion was lesioned to destroy all ascending input to the brain from the thoracic and abdominal ganglia (including the MDHns; see dashed line in Fig. 2D) or the divide between the mesothoracic and metathoracic ganglia was cut to destroy all ascending cells posterior to the mesothoracic ganglia (MsG; including pairs of HA cells in the metathoracic ganglia and the first two abdominal ganglia; see dashed line between the MsG and the MtG in Fig. 2F). Moths were fed sugar water each day following the ablation to increase survival rates. After 8 days, the brains were dissected for immunolabeling for HA-ir and brp-ir. For the ablation of the connective between the prothoracic ganglion and SEZ we used 6 moths in which we cut the connective between the prothoracic ganglion and the SEZ and 6 sham operated moths. Successful ablation was verified by a lack of HA-ir in the remnants of the connective, while sham ablation (when the connective was not cut) was verified by the presence of HA-ir in the remnants of the connective. For the ablation of the boundary between the mesothoracic and metathoracic ganglia, successful ablation was verified by a lack of HA-ir fibers in the mesothoracic ganglion that originate from the more posterior ganglia. In 10 moths, 2 moths resulted in the successful elimination of the ascending fibers from the metathoracic ganglion, but this did not result in loss of HA-ir in the AL.

\section{Confocal Microscopy}

Optical stacks were acquired using an Olympus Fluoview FV 1000 confocal microscope. All scans were taken with either a 20X or $40 \mathrm{X}$ oil lens. Confocal planes were stacked with optimized step sizes for the given objective (1.79 um for 20X and 0.54 um for 40X) in the Fluoview viewer software (FV10-ASW Version 04.00.02.09). All images were scanned at either 512x512 or $1024 \times 1024$ pixel resolution. Cell body counts and size measurements were performed in Fluoview. Corel Draw (Version 13.0.0.576) was used to organize figures. Vaa3D (Peng et al., 
2010) was used to generate 3D reconstructions of confocal stacks that could be rotated to resolve the degree to which structures physically overlap.

\section{Results}

\section{Two HA immunoreactive cells project from the MsG to the AL}

Although motor-to-sensory circuits have been extensively characterized in many sensory systems, there is a dearth of detailed descriptions of input from motor to olfactory centers. The purpose of this study was to extensively characterize the structure, candidate targets and development of a motor-to-olfactory circuit. In Manduca a pair of HA-ir cells (the MDHns) project from the MsG to the AL (Homberg, 1994). However, there is very little known about the fine morphological details of MDHns in either the MsG or the AL. Furthermore, nothing is known about the potential targets of the MDHns or their development through metamorphosis. Figure 2 shows the MDHns in the nervous system including the brain (Fig. 2A), entering the AL (Fig. 2B), entering the SEZ from the neck connective (Fig. 2C), in the neck connective (Fig. 2D), in the prothoracic ganglion (Fig. 2E), and in the MsG (Fig. 2F; n=54).

The large MDHn cell bodies ( $60 \mu \mathrm{m}$ in diameter) are located on the ventral surface of the MsG (Fig. 3A) near the intersection of the sagittal and coronal midlines, and extend large primary neurites to the dorsal MsG (Fig. 3A; $n=30$ ). In the dorsal MsG, the MDHns produce a radial planar sheet of processes, with occasional sparse innervation of the ventral MsG (Fig. 3B). Each MDHn extends a single axon ipsilaterally through the prothoracic ganglion and SEZ (Fig. 2E, 3A, 3B), and bilaterally arborizes in the ventral AL (Fig. 2A; 4A). To determine the extent to which the MDHns innervate the AL, we used the BRP antibody to delineate glomerular boundaries and immuno-labeled for HA. Varicose HA-ir processes extensively innervate a subset of ventral posterior glomeruli (Fig. 4A,B; n=21) and extend sparsely into the ventral posterior coarse neuropil of the AL. Reconstructing and rotating the confocal image stack confirms that the HA-ir processes both encapsulate and innervate the glomeruli (Fig. 4C,D). There is not much known about the ventral glomeruli in Manduca other than CO2 being processed in the LPOG (Guerenstein et al., 2004), therefore why the MDHns are restricted to this area of AL is unclear.

In addition to the MDHns, HA-ir neurons in the metathoracic and first abdominal ganglia (Fig. 2F) extend processes to the brain via the cervicothoracic connectives. The processes of these HA-ir from other ganglia intertwine with those from the MDHn in the prothoracic ganglia (Fig. 2E), making it difficult to definitively ascribe the HA-ir processes in the AL as belonging exclusively to the MDHns. Furthermore, there are 20 pairs of HA-ir neurons in the SEZ and protocerebrum of Manduca (Homberg and Hildebrand, 1991). To demonstrate that the HA-ir processes in the AL originate from the MDHns, we performed two ablation experiments (Fig. $4 \mathrm{E}, \mathrm{F})$. In the first experiment, we cut the cervicothoracic connective between the prothoracic ganglion and brain in adult moths and kept the moths alive for 8 days. This protocol eliminates HA-ir processes arising from cells in the thoracic and abdominal ganglia (including the MDHns), but leaves the processes from other HA-ir neurons in the brain intact (notice HA-ir ventral to the AL outlined by dotted line with no HA-ir overlapping with BRP-ir outlining glomeruli). Ablation of thoracic and abdominal sources of HA-ir was confirmed via elimination of HA-ir entering the ventral SEZ. Ablating the cervicothoracic connective eliminates all HA-ir in the AL (Fig. 4E) indicating that the $\mathrm{HA}$-ir processes in the AL originate from the ventral nerve cord, posterior to 
the cut site. It is possible that cutting the cervicothoracic connectives indirectly affects other HAir neurons in the brain, which might contribute to AL HA-ir processes we observe. However, we find no evidence to support this notion. In the second ablation experiment, we lesioned the thoracic ganglia at the boundary between the metathoracic ganglion and MsG. This ablates all ascending HA-ir processes posterior to the MDHns (i.e. the HA-ir cells in the metathoracic and abdominal ganglia) but leaves MDHn processes intact. These experiments show that after ablating the cells posterior to the MDHns that there is still HA-ir in the AL (Fig. 4F). Together these experiments suggest that the MDHns are the exclusive source of the HA-ir processes in the AL.

\section{The MsHisClB receptor is expressed in a subset of GABAergic LNs, one FMRFaminergic $L N$ and one allatotropinergic $L N$.}

To determine the candidate targets of the MDHns, antibodies were generated against the Manduca homolog of the HA B-type receptor (MsHisClB; Fig. 1 and see Methods). Insects possess two HA receptor types, HisClA and HisClB (Gisselman et al., 2002; Zheng et al., 2002), both of which are ligand-gated chloride channels (McClintock and Ache, 1989; Hardie, 1989). Each receptor is homomeric with two genes coding for the two subunits HisCl- $\alpha 1$ and HisCl- $\alpha 2$ (Gisselmann et al., 2001). These receptors are members of the large cys-bridge superfamily of ligand-gated ion channels comprised of four transmembrane domains (Gisselmann et al., 2001). The MsHisCIB antibody produces extensive labeling in the lamina of the optic lobes of Manduca where histaminergic photoreceptors terminate (Fig. 1G) which is consistent with HisClB receptor expression by glial cells in the lamina of Drosophila (Pantazis et al., 2008). Within the AL, MsHisClB-ir was observed in every glomerulus, which was surprising as the MDH neurons only innervate a set of ventral glomeruli. The MsHisClB antibody produces only a single band in western blots at the predicted height for the MsHisClB receptor (Fig. 1F; $\mathrm{n}=5$ ) and all labeling is eliminated by pre-adsorption with the immunogenic sequence (Fig. $1 \mathrm{G}, \mathrm{H} ; \mathrm{n}=6$ ), making it unlikely that this antibody is labeling additional proteins. It is however, possible that the MsHisClB-ir reflects distribution of the $\mathrm{MsHisClB}$ receptor during transport throughout the cell as opposed to distribution of the receptor at functional synapses.

In the AL we observed 11 ( $\pm 0.81 \mathrm{SEM}$, from 3 moths) and 9.3 ( $\pm 0.43 \mathrm{SEM}$, from 3 moths) MsHisClB-ir cell bodies in males and females, respectively, in the lateral cell cluster (Fig. 5A). The sex differences observed may be due to neurons that project to the macroglomerular complex in males, as we see widespread labeling therein (Fig. 5A). We observed two classes of MsHisClB labeled cells differing in cell body size. In each AL there were 1-2 larger MsHisClB-ir cells (23.98 um \pm 0.73 SEM diameter; $n=10)$ with the remainder having smaller cell bodies $(14.79 \mathrm{um} \pm 0.52$ SEM diameter; $\mathrm{n}=10$ ). $\mathrm{LN}$ cell bodies are found only in the lateral cell cluster and fall within in two populations based on cell body size being either $\sim 12$ ums or $\sim 20$ ums in diameter (Hoskins et al., 1986) whereas we calculate an average PN cell body size of 8.16 um ( \pm 0.16 SEM) from our retrogradely filled PNs, thus the size of MsHisClB-ir cell bodies is consistent with LNs. Furthermore, we do not observe any MsHisClB-ir processes within any of the AL output tracts (Fig. S1A) and there are no HA-ir processes innervating any of the AL output tracts (Fig. S1B). The MsHisClB-ir neurons collectively branch in every glomerulus (Fig. 5A; n=37), again consistent with the MsHisClB receptor being expressed by LNs, rather than PNs. To further functionally characterize these MsHisClB-ir cells, we co-labeled for several transmitters, including GABA (Hoskins et al., 1986), FMRFamide (Homberg et al., 1990), and ATR (Utz et al., 2006). All but one MsHisClB-ir labeled neuron was GABA-ir (Fig. 5B; n=19) with one cell co-labeled 
for MsHisClB and FMRFamide and one cell co-labeled for MsHisClB and ATR (Fig. 5E,H respectively; $n=5,10$ respectively). Together these results suggest that any influence of the MDHns on AL processing is exerted via a population of GABAergic and peptidergic LNs. The expression of the MsHisClB receptor by AL neurons and the MDHn being the sole source of HA-ir in the AL suggests that the MDH neurons provide some form of input to the AL. This does not, however, imply that the MDH neurons do not also provide input to circuitry within the MsG. MsHisClB receptor is also expressed within the $\mathrm{MsG}(\mathrm{S} 2)$, however both the MDHns and HA-ir neurons from the metathoracic and abdominal ganglion (Fig. 2F) innervate the MsG, suggesting that HA also plays a role in network function within the $\mathrm{MsG}$.

\section{MDHns survive metamorphosis but the LAC lacks MsHisClB expression.}

There are many neurons that survive metamorphosis, often being repurposed to take on new tasks to match the dramatic changes in behavioral demands between the larval and adult life stage. In Manduca, motor neurons survive metamorphosis, but their morphology and biophysical properties are altered dramatically to allow them to take on life-stage specific tasks, for instance, transitioning from participating in walking motor programs as larvae to flying motor programs as adults (Duch and Levine 2000). Given that odor-guided flight is an adult specific behavior, we predicted that the MDHns would either not be present or the MsHisClB-ir would not be expressed in the LAC. Similar to adults (see Fig. 3A), fifth instar larvae have a pair of large HA-ir cells in the MsG that ascend to the brain (Fig. 6A). As in adults, the cell bodies are also located ventrally near the intersection of the sagittal and horizontal midlines of the MsG, with a single axon ipsilaterally projecting up each connective. Furthermore, the HA-ir processes also radiate in all directions in the dorsal MsG as in the adult. Because the LAC does not express BRP-ir, we used Syto-59 to label the nuclei of cell bodies that surround the LAC (Fig. 6B,C) as a means of highlighting the boundaries of this brain region. In the larval brain, HA-ir is most abundant in the tritocerebrum (Fig. 6B; dash line) just ventral and lateral to the larval LAC (small dotted line) with a small amount of HA-ir entering the LAC $(n=17)$. This suggests that the MDHns are present and project to the olfactory system of larval Manduca. However, there are no MsHisClB-ir neurons within the LAC, despite the presence of MsHisClB-ir collaterals in the tritocerebrum (Fig. 6C; $\mathrm{n}=6$ ). This suggests that while the MDHns provide sparse innervation of the LAC, they likely do not play a functional role in the larval olfactory system, at least via the MsHisClB receptor, although it is possible that the MsHisClA receptor is expressed there. What role this circuit would play in the larval olfactory system is not clear as the larva do not fly, but there could be information pertaining to walking patterns.

\section{Discussion}

Animals use a variety of behavioral strategies to optimize internal representations of the external world, including repetitive motor patterns that alter stimulus structure. Nervous systems have concurrently evolved circuits that provide information to sensory systems about impending behaviors that will affect sensory input. Although this has been well-documented in many sensory systems, very little is known about neural circuits projecting from neural centers governing odorguided behaviors to olfactory networks. The goal of this study was to characterize a novel sensorymotor to olfactory circuit that projects from flight sensory-motor centers to the primary olfactory processing center in insects. We found that the MDH circuit provides the only source of HA to the AL and affects a small but diverse population of widely projecting LNs in adult Manduca (Fig. 7). 
Our data suggest that the MDHns provide histaminergic inhibitory input to the AL that could modify olfactory processing within the context of flight or other MsG mediated activity such as walking patterns.

The MDHn processes project laterally across the MsG (Fig. 3A), yet are most dense in the dorsal MsG (Fig. 3B), suggesting that while they may integrate information from both sides of the animal, they are likely to interact with cells that are restricted to the dorsal aspect of the MsG. The MsG contains wing and leg motor neurons, sensory afferents, CPG components and modulatory neurons some of which occupy specific MsG regions. The dendritic fields of wing elevator and depressor motor neurons are located in the dorsal region of the MsG in Manduca (Rind, 1983) whereas most of the sensory afferents from the wings are localized in both the dorsal and ventral MsG in a closely related species of hawkmoth, Agrius convolvuli (Ando et al., 2011). In addition, there are a population of non-spiking, GABAergic local interneurons that project to the dorsal side of the MsG of the locust (Watson and Burrows 1987), and populations of octopaminergic (Stevenson et al. 1992), serotonergic and dopaminergic neurons (Claassen and Kammer, 1986) that project throughout the MsG. The extensive branching of the MDHns in the MsG suggests that these neurons interact with one or more components of the MsG. The potential cumulative effect of multiple inputs onto MDHns makes understanding the input to this neural circuit challenging. Single neurons releasing multiple neurotransmitters alone can have state dependent effects on network output (Nusbaum et al., 2001; Swensen and Marder, 2000). Furthermore, this complexity is compounded when considering the MDHns impact a heterogeneous population of AL LNs.

Arthropod HA receptors are ligand gated $\mathrm{Cl}^{-}$channels (McClintock and Ache, 1989; Hardie, 1989) sharing $45 \%$ amino acid similarity to the alpha3 subunit of the human glycine receptor (Zheng et al., 2002), thus the effect of HA on MsHisClB expressing LNs is likely inhibitory in nature. Within the AL there are $~ 300 \mathrm{LNs}$ that belong to a diverse set of subtypes based on morphology, neurotransmitter content and physiological response properties (Chou et al., 2011; Reisenman et al., 2011). These LNs mediate diverse processing mechanisms such as lateral inhibition for gain control (Olsen and Wilson, 2007). In addition, these widely branching LNs activate metabotropic receptors whose effects occur on longer and more variable time scales than ionotropic receptors. Therefore the overall network effect of MDHn activity is variable in both the spatial and temporal domain making this circuit difficult to characterize. One potential mechanism would be suppression of GABA, FMRFamide and ATR release by select LNs within the AL. Theoretically, decreasing the influence of these predominantly inhibitory LNs could act to disinhibit the inhibitory AL local network, which could lead to a refinement of PN activity. While the role this refinement has on AL output activity is not clear, it could be in response to the rapid oscillatory nature of the stimulus experience which is driven in part by wing-beating (Sane and Jacobson, 2006). Finally, while invertebrate sensory-motor to sensory circuits typically function to filter reafferent stimuli, we suggest that it is unlikely that the MDHns function in this manner because non-olfactory responses persist in fully intact preparations (Tripathy et al., 2010). Therefore, it may be that MDHn activity indirectly refines PN spatiotemporal response patterns to modify the information output to higher order processing centers during flight. Indeed evidence suggests that the fine temporal structure of AL/OB output patterns contain substantial information about odor identity (Rebello et al., 2014; Staudacher et al., 2009; Daly et al., 2004). However, future studies investigating both the activity patterns of MDHns during flight behavior and the consequences of HA release on AL response properties are necessary to confirm this hypothesis. 
Many active sampling behaviors rapidly sample the sensory field providing discrete epochs of input to a sensory system; for example, micro-saccadic eye movements mentioned above. In addition, the details of temporally structured reafference may be dependent on the behavior of the animal. For instance, when exposed to a novel stimulus mice and rats increase their sniff frequencies (Wesson et al., 2008a; Kepecs et al., 2007) and sniff frequency modulation is dependent on the specifics of the behavioral task such as free exploration, detection, and discrimination (Wesson et al., 2008b). Insects also show stereotyped active sampling behaviors that are temporally structured. Bombyx mori require wing beating to track pheromone plumes despite their inability to fly (Obara, 1979) and male oriental fruit moths continue to fan their wings as they track a calling female even though their final approach is on foot (Baker and Carde, 1979).

From a whole nervous system perspective, it is perhaps not surprising that network-specific processing of information must be adjusted based on inputs from many disparate networks. It is becoming increasingly apparent that networks receive input from a large number of different sources and thus must integrate a variety of ongoing contexts. The mammalian Raphe nuclei provide widespread serotonergic input, yet they also receive input from many other brain areas (Dorocic et al., 2014; Liu et al, 2014; Weissbourd et al 2014). More specifically, the olfactory systems of animals receive a variety of inputs from other brain regions including serotonergic (Kent et al., 1987; McLean et al., 1987; Dacks et al., 2006), dopaminergic (Dacks et al., 2012), cholinergic (Macrides et al., 1981; Mandairon et al., 2006), octopaminergic (Dacks et al., 2005, Sinakevitch et al., 2005; Sinakevitch and Strausfeld, 2006; Dacks and Nighorn, 2011) and GABAergic (Nunez-para et al., 2013; Garcia-Llanes et al., 2010) cells all of which modify sensory processing within different, sometimes competing contexts. Our data support the hypothesis that olfactory processing in Manduca may also be adjusted within the context of ongoing activity in the MsG via the histaminergic MDHns.

\section{References}

Adrian,ED. 1942. Olfactory reactions in the brain of the hedgehog. The Journal of Physiology 100:459-473.

Ando N, Wang H, Shirai K, Kanzaki R. 2011. Central projections of the wing afferents in the hawkmoth, Agrius convolvuli. J insect phys 57:1518-1536.

Baker TC, Carde RT. 1979. Analysis of pheromone-mediated behaviors in male Grapholitha molesta, the oriental fruit moth (Lepidoptera: Tortricidae). Environ Entomol 8:956-968.

Bell CC. 1981. An efference copy which is modified by reafferent input. Science 214:450-453.

Bell RA, Joachim FG. 1976. Techniques for rearing laboratory colonies of tobacco hornworms and pink bollworms. Ann Entomol Soc Am 69:365-373.

Berg BG, Schachtner J, Homberg U. 2009. Gamma-aminobutyric acid immunostaining in the antennal lobe of the moth Heliothis virescens and its colocalization with neuropeptides. Cell Tissue Res 335:593-605.

Brainard MS, Doupe AJ. 2000. Interruption of a basal ganglia-forebrain circuit prevents plasticity of learned vocalizations. Nature 404:762-766. 
Chalfie M, Sulston JE, White JG, Southgate E, Thomson JN, Brenner S. 1985. The neural circuit for touch sensitivity in Caenorhabditis elegans. J Neurosci 5:956-964.

Chou YH, Spletter ML, Yaksi E, Leong JC, Wilson RI, Luo L. 2010. Diversity and wiring variability of olfactory local interneurons in the Drosophila antennal lobe. Nature neuroscience. 13:439-449.

Christensen TA, Waldrop BR, Hildebrand JG. 1998. Multitasking in the olfactory system: context-dependent responses to odors reveal dual GABA-regulated coding mechanisms in single olfactory projection neurons. J Neurosci 18:5999-6008.

Claassen DE, Kammer AE. 1986. Effects of octopamine, dopamine, and serotonin on production of flight motor output by thoracic ganglia of Manduca sexta. J Neurobiol 17:1-14.

Clustering CC, Kittel RJ, Wichmann C, Rasse TM, Fouquet W, Schmidt M, Schmid A, Wagh D A, Pawlu C, Kellner RR, Willig KI, Hell SW, Buchner E, Heckmann M, Sigrist SJ. 2006. Bruchpilot promotes active zone assembly, Ca2+ channel clustering, and Vesicle Release. Science 312:1051-1054.

Crapse TB, Sommer MA. 2008. Corollary discharge across the animal kingdom. Nature Reviews Neuroscience 9:587-600.

Dacks AM, Christensen TA, Agricola HJ, Wollweber L, Hildebrand JG. 2005. Octopamineimmunoreactive neurons in the brain and subesophageal ganglion of the hawkmoth Manduca sexta. J Comp Neurol. 488:255-68.

Dacks AM, Christensen TA, Hildebrand JG. 2006. Phylogeny of serotonin-immunoreactive neuron in the primary olfactory center of the insect brain. J Comp Neurol 498:727-746.

Dacks AM, Nighorn AJ. 2011. The organization of the antennal lobe correlates not only with phylogenetic relationship, but also life history: a Basal hymenopteran as exemplar. Chem Senses 36:209-20.

Dacks AM, Reisenman CE, Paulk AC, Nighorn AJ. 2010. Histamine-immunoreactive local neurons in the antennal lobes of the hymenoptera. J Comp Neurol 518:2917-2933.

Dacks AM, Riffell JA., Martin JP, Gage SL, Nighorn AJ. 2012. Olfactory modulation by dopamine in the context of aversive learning. J Neurophysiol 108:539-550.

Daly KC, Galan RF, Peters OJ, Staudacher EM. 2011. Detailed characterization of local field potential oscillations and their relationship to spike timing in the antennal lobe of the moth Manduca sexta. Front Neuroeng 4:1-22.

Daly KC, Kalwar F, Hatfield M, Staudacher EM, Bradley SP. 2013. Odor detection in Manduca sexta is optimized when odor stimuli are pulsed at a frequency matching the wing beat during flight. PLoS One 8:1-18. 
Daly KC, Wright GA, Smith BH. 2004. Molecular features of odorants systematically influence slow temporal responses across clusters of coordinated antennal lobe units in the moth Manduca sexta. Journal of neurophysiology 92:236-254.

Dorocic IP, Fürth D, Xuan Y, Johansson Y, Pozzi L, Silberberg G, Carlén M, Meletis K. 2014. A Whole-Brain Atlas of Inputs to Serotonergic Neurons of the Dorsal and Median Raphe Nuclei. Neuron 83:663-678.

Duch C, Levine RB. 2000. Remodeling of membrane properties and dendritic architecture accompanies the postembryonic conversion of a slow into a fast motoneuron. J Neurosci 20:6950-6961.

Fouquet W, Owald D, Wichmann C, Mertel S, Depner H, Dyba M, Hallermann S, Kittel RJ, Eimer S, Sigrist SJ. 2009. Maturation of active zone assembly by Drosophila Bruchpilot. J Cell Biol 186:129-145.

Guerenstein, PG, Christensen TA, Hildebrand JG. 2004. Sensory processing of ambient CO2 information in the brain of the moth Manduca sexta. Journal of Comparative Physiology A. 190:707-725.

Gracia-Llanes FJ, Crespo C, Blasco-Ibáñez JM, Nacher J, Varea E, Rovira-Esteban L, MartínezGuijarro FJ. 2010. GABAergic basal forebrain afferents innervate selectively GABAergic targets in the main olfactory bulb. Neuroscience 170:913-922.

Halpern BP. 1983. Tasting and smelling as active, exploratory sensory processes. Am J Otolaryngol 4:246-249.

Hardie RC. 1989. A histamine-activated chloride channel involved in neurotransmission at a photoreceptor synapse. Nature 339:704-706.

Hernández PP, Olivari F a, Sarrazin AF, Sandoval PC, Allende ML. 2007. Regeneration in zebrafish lateral line neuromasts: expression of the neural progenitor cell marker sox 2 and proliferation-dependent and-independent mechanisms of hair cell renewal. Dev Neurobiol 67:637-654.

Holst E, Mittelstaedt H. 1950. The principle of reafference: Interactions between the central nervous system and the peripheral organs. Die Naturwissenschaften 37:464-476.

Homberg U. 1994. Distribution of neurotransmitters in the insect brain. Gustav Fischer Verlag.

Homberg U, Hildebrand JG. 1991. Histamine-immunoreactive neurons in the midbrain and suboesophageal ganglion of the sphinx moth Manduca sexta. Journal of comparative neurology. 307:647-657.

Hoskins SG, Homberg U, Kingan TG, Christensen T a., Hildebrand JG. 1986. Immunocytochemistry of GABA in the antennal lobes of the sphinx moth Manduca sexta. Cell Tissue Res 244:243-252. 
Houot B, Burkland R, Tripathy S, Daly KC. 2014. Antennal lobe representations are optimized when olfactory stimuli are periodically structured to simulate natural wing beat effects. Front Cell Neurosci 8:159.

Jones AK, Bera AN, Kees K, Sattelle DB. 2010. The cys-loop ligand-gated ion channel gene superfamily of the parasitoid wasp, Nasonia vitripennis. Heredity 3:247-259.

Kennedy JS, Ludlow AR, Sanders CJ. 1981. Guidance of flying male moths by wind-borne sex pheromone. Physiol Entomol 6:395-412.

Kent KS, Hoskins SG, Hildebrand JG. 1987. A novel serotonin-immunoreactive neuron in the antennal lobe of the sphinx moth Manduca sexta persists throughout postembryonic life. Dev Neurobiol 18:451-465.

Kepecs A, Uchida N, Mainen ZF. 2007. Rapid and precise control of sniffing during olfactory discrimination in rats. J Neurophysiol 98:205-213.

Liu Z, Zhou J, Li Y, Hu F, Lu Y, Ma M, Feng Q, Zhang JE, Wang D, Zeng J, Bao J, Kim JY, Chen ZF, El Mestikawy S, Luo M. 2014. Dorsal raphe neurons signal reward through 5-HT and glutamate. Neuron 81:1360-74.

Lizbinski KM, Metheny JD, Bradley SP, Kesari A, Dacks AM. In Press. The Anatomical Basis for Modulatory Convergence in the Antennal Lobe of Manduca sexta. J Comp Neurol. In Press.

Loudon C, Best B, Koehl M. 1994. When does motion relative to neighboring surfaces alter the flow through arrays of hairs? J Exp Biol 193:233-54.

Loudon C, Koehl MA. 2000. Sniffing by a silkworm moth: wing fanning enhances air penetration through and pheromone interception by antennae. J Exp Biol 203:2977-2990.

Mandairon N, Ferretti CJ, Stack CM, Rubin DB, Cleland T a., Linster C. 2006. Cholinergic modulation in the olfactory bulb influences spontaneous olfactory discrimination in adult rats. Eur J Neurosci 24:3234-3244.

Martinez-Conde S, Macknik SL, Hubel DH. 2002. The function of bursts of spikes during visual fixation in the awake primate lateral geniculate nucleus and primary visual cortex. Proc Natl Acad Sci USA 99:13920-13925.

Martinez-Conde S, Macknik SL, Troncoso XG, Dyar TA. 2006. Microsaccades counteract visual fading during fixation. Neuron 49:297-305.

Martinez-conde S, Martinez-conde S, Macknik SL, Macknik SL, Hubel DH, Hubel DH. 2000. Microsaccadic eye movements and firing of single cells in the striate cortex of macaque monkeys. Nat Neurosci 3:409.

McClintock TS, Ache BW. 1989. Histamine directly gates a chloride channel in lobster olfactory receptor neurons. Proc Natl Acad Sci USA 86:8137-8141. 
McLean JH, Shipley MT. 1987. Serotonergic afferents to the rat olfactory bulb: I. Origins and laminar specificity of serotonergic inputs in the adult rat. J Neurosci 7:3016-3028.

Melzig J, Buchner S, Wiebel F, Wolf R, Burg M, Pak WL, Buchner E. 1996. Genetic depletion of histamine from the nervous system of Drosophila eliminates specific visual and mechanosensory behavior. J Comp Physiol A 179:763-773.

Mohr C, Roberts PD, Bell CC. 2003. The mormyromast region of the mormyrid electrosensory lobe. II. Responses to input from central sources. J Neurophysiol 90:1211-1223.

Nunez-Parra A, Maurer RK, Krahe K, Smith RS, Araneda RC. 2013. Disruption of centrifugal inhibition to olfactory bulb granule cells impairs olfactory discrimination. PNAS 110:14777-82.

Nusbaum MP, Blitz DM, Swensen AM, Wood D, Marder E. 2001. The roles of co-transmission in neural network modulation. Trends in neurosci 24:146-154.

Obara T. 1979. Bombyx mori mating dance: an essential in locating the female. Appl Entomol Zool 14:130-132.

Pantazis A, Sezaran A, Liu C-H, Nikolaev A, Rister J, Thum AS, Roeder T, Semenov E, Juusola M, Hardie RC. 2008. Distinct roles for two histamine receptors (hclA and hclB) at the Drosophila photoreceptor synapse. J Neurosci 28:7250-7259.

Peng H, Ruan Z, Long F, Simpson JH, Myers EW. (2010). V3D enables real-time 3D visualization and quantitative analysis of large-scale biological image data sets. Nature biotechnology. 28:348-353.

Porter J, Craven B, Khan RM, Chang S-J, Kang I, Judkewitz B, Volpe J, Settles G, Sobel N. 2007. Mechanisms of scent-tracking in humans. Nat Neurosci 10:27-29.

Poulet JF, Hedwig B. 2002. A corollary discharge maintains auditory sensitivity during sound production. Nature 418:872-876.

Rebello MR, McTavish TS, Willhite DC, Short SM, Shepherd GM, Verhagen JV. 2014. Perception of Odors Linked to Precise Timing in the Olfactory System. PLoS biology 12.

Reinagel P, Godwin D, Sherman SM, Koch C. 1999. Encoding of visual information by LGN bursts. J Neurophysiol 81:2558-2569.

Reisenman CE, Dacks AM, \& Hildebrand JG. 2011. Local interneuron diversity in the primary olfactory center of the moth Manduca sexta. Journal of Comparative Physiology A, 6:653-665.

Rind CF. 1983. The organization of flight motoneurones in the moth, Manduca sexta. J Exp Biol 102:239-251.

Ross J, Concetta Morrone M, Goldberg ME, Burr DC. 2001. Changes in visual perception at the time of saccades. Trends in Neurosciences 24:113-121. 
Rothermel M, Carey RM, Puche a, Shipley MT, Wachowiak M. 2014. Cholinergic inputs from basal forebrain add an excitatory bias to odor coding in the olfactory bulb. J Neurosci 34:46544664.

Sane SP, Jacobson NP. 2006. Induced airflow in flying insects II. Measurement of induced flow. J Exp Biol 209:43-56.

Sievers F, Wilm A, Dineen DG, Gibson TJ, Karplus K, Li W, Lopez R, McWilliam H, Remmert M, Söding J, Thompson JD, Higgins D. 2011. Fast, scalable generation of high-quality protein multiple sequence alignments using Clustal Omega. Mol Syst Biol 7:1-6.

Sinakevitch I, Niwa M, Strausfeld NJ. 2005. Octopamine-like immunoreactivity in the honey bee and cockroach: comparable organization in the brain and subesophageal ganglion. J Comp Neurol. 488:233-54.

Sinakevitch I, Strausfeld NJ. 2006. Comparison of octopamine-like immunoreactivity in the brains of the fruit fly and blow fly. J Comp Neurol. 494:460-75.

Sommer MA, Wurtz RH. 2002. A pathway in primate brain for internal monitoring of movements. Science 296:1480-1482.

Staudacher EM, Huetteroth W, Schachtne, J, Daly KC. 2009. A 4-dimensional representation of antennal lobe output based on an ensemble of characterized projection neurons. Journal of neuroscience methods 180:208-223.

Stevenson PA, Pflüger HJ, Eckert M, Rapus J. 1992. Octopamine immunoreactive cell populations in the locust thoracic-abdominal nervous system. J Comp Neurol 315:382-397.

Stuart AE. 1999. From fruit flies to barnacles, histamine is the neurotransmitter of arthropod photoreceptors. Neuron 22:431-433.

Swensen AM, Marder E. 2000. Multiple peptides converge to activate the same voltagedependent current in a central pattern-generating circuit. J Neurosci 20: 6752-6759.

Tripathy SJ, Peters OJ, Staudacher EM, Kalwar FR, Hatfield MN, Daly KC. 2010. Odors pulsed at wing beat frequencies are tracked by primary olfactory networks and enhance odor detection. Front Cell Neurosci 4:1-14.

Utz S, Huetteroth W, Vömel M, Schactner J.2008. Mas-allatotropin in the developing antennal lobe of the sphinx moth Manduca sexta: distribution, time course, developmental regulation, and colocalization with other neuropeptides. Devel Neurobiology 68:123-142.

Veenstra JA, Hagedorn HH. 1993. Sensitive enzyme immunoassay for Manduca allatotropin and the existence of an allatotropin-immunoreacitve peptide in Periplaneta americana. Arc of insect biochem and phys 23:99-109.

Wagh D a., Rasse TM, Asan E, Hofbauer A, Schwenkert I, Dürrbeck H, Buchner S, Dabauvalle MC, Schmidt M, Qin G, Wichmann C, Kittel R, Sigrist SJ, Buchner E. 2006. Bruchpilot, a 
protein with homology to ELKS/CAST, is required for structural integrity and function of synaptic active zones in Drosophila. Neuron 49:833-844.

Watson AH, Burrows M. 1987. Immunoctochemical and pharmacological evidence for GABAergic spiking local interneurons in the locust. J Neurosci 7:1741-1751.

Weiss KR, Cohen JL, Kupfermann I. 1978. Modulatory control of buccal musculature by a serotonergic neuron (metacerebral cell) in Aplysia. J Neurophysiol 41:181-203.

Weissbourd B, Ren J, DeLoach KE, Guenthner CJ, Miyamichi K, Luo L. 2014. Presynaptic partners of dorsal raphe serotonergic and GABAergic neurons. Neuron 83:645-62.

Wesson DW, Carey RM, Verhagen J V., Wachowiak M. 2008a. Rapid encoding and perception of novel odors in the rat. PLoS Biol 6:717-729.

Wesson DW, Donahou TN, Johnson MO, Wachowiak M. 2008b. Sniffing behavior of mice during performance in odor-guided tasks. Chem Senses 33:581-596.

Witten JL, Truman JW. 1996. Developmental plasticity of neuropeptide expression in motoneurons of the moth, Manduca sexta: steroid hormone regulation. J Neurobio. 29:99-114.

Woo SH, Stumpfova M, Jensen UB, Lumpkin EA, Owens, D M. 2010. Identification of epidermal progenitors for the Merkel cell lineage. Development. 23:3965-3971.

Zaretsky M, Rowell CHF. 1979. Saccadic suppression by corollary discharge in the locust. Nature. 280: 583-585.

Zheng Y, Hirschberg B, Yuan J, Wang AP, Hunt DC, Ludmerer SW, Schmatz DM, Cully DF. 2002. Identification of two novel Drosophila melanogaster histamine-gated chloride channel subunits expressed in the eye. J Biol Chem. 3:2000-2005. 


\section{Figure legends}

Figure 1. Characterization of the histamine (HA) GABA and Manduca sexta histamine B receptor (MsHisClB) antibodies. (A) HA labeling in control animals where the antibody was not preadsorbed. Arrow head highlights HA labeling that enters the ventral and medial AL (for all panels outlined with a dashed line). (B) HA labeling in the antennal lobe (AL) is abolished after the HA antibody was pre-adsorbed with a 10:1 HA to antibody solution.(C) GABA labeling remains in control animals where the antibody was not pre-adsorbed with GABA. (D) GABA labeling in the AL is abolished after the GABA antibody was pre-adsorbed with a 10:1 GABA to antibody solution. For each panel the same dorsal lateral axis is used. (E) Amino acid sequence alignment of the histamine B receptor subunits of Manduca sexta (MsHB; Msex2.04603-RA), Drosophila melanogaster (DmHB; ACA13298.1), Apis mellifera (AmHB; ABG75740.1) and Nasonia vitripennis (NvHB; ACZ51422.1). Asterisks indicate sequence identity across all 4 species. Bold font indicates the immunogenic peptide sequence from Manduca that was used to generate the MsHisClB antibody. (F) Western blot using MsHisClB receptor antibody on Manduca brain tissue resulted in a single band at the predicted molecular weight $(36 \mathrm{kDa})$ of the MsHisClB protein. (G) Frontal section of optic lobe depicting MsHisClB-ir in the lamina (as labeled by an asterisks). (H) Pre-adsorption with the immunogenic peptide sequence eliminates all labeling in the lamina. Scale bars $=50 \mu \mathrm{m} . \mathrm{D}=$ dorsal, $\mathrm{L}=$ lateral, $\mathrm{A}=$ anterior.

Figure 2. MDH neurons project from the mesothoracic ganglia to the AL of Manduca sexta. (A) Frontal view of HA-ir labeling in a whole mount brain preparation. Hatched line outlines the AL boundary. (B) Saggital view of a HA-ir process entering the AL (bracket). (C) Frontal view of HA-ir processes entering the SEZ from the cervicothoracic connective. Notice that 4 pairs enter the SEZ. (D) HA-ir processes in the cervicothoracic connective. Brackets highlight three HA-ir processes. (E) Horizontal view of the HA-ir processes in the prothoracic ganglia. Notice 4 pairs ascending from here as well. (F) Horizontonal view of HA-ir in the MsG, the metathoracic ganglia, and the first two abdominal ganglia. Each SEZment has a pair of HA-ir cell bodies located in the medial third of their respective ganglia. (G) Schematic of the Manduca nervous system highlighting the MDHns (green). Hatched boundary indicates the MsG. All scale bars = $100 \mathrm{um}$. $\mathrm{AL}=$ antennal lobe; ef=esophageal foramen; SEZ=sub-esophageal zone; CTC=cervicothoracic connective; $\mathrm{PtG}=$ prothoracic ganglia; $\mathrm{MsG}=$ mesothoracic ganglia; $\mathrm{MtG}=$ metathoracic ganglia; ab1=abdominal ganglion 1; ab2=abdominal ganglion 2 .

Figure 3. MDH neurons processes radiate laterally throughout the $\mathrm{MsG}$, but are primarily restricted to the dorsal aspect. (A) Horizontal view of the MSG showing two cell bodies with each cell projecting out one side of the ganglia. (B) Sagittal section of the MsG shows two large HA-ir cells with cell bodies (white arrow head) situated ventrally and a radiating dendritic field dorsally with the axon (black arrow) projecting up the connective between the mesothoracic and prothoracic ganglia. White dotted line indicates the boundary between the mesothoracic and metathoracic ganglia. Arrow indicates MDHn cell body in each image. All scale bars $=100 \mu \mathrm{m}$.

Figure 4. The MDH neurons provide the sole source of HA-ir input to the ALs. (A) Saggital section of the AL with HA-ir (green). Bruchpilot (Brp) (magenta) outlines glomeruli of the AL. Dotted line outlines the posterior boundary of the AL. Scale bar $=100 \mathrm{um}$. (B) High magnification view of inset from (A). Highly varicose HA-ir processes innervate 4-6 ventral posterior glomeruli. Scale bar $=50 \mathrm{um}$. (C) Rotation of image (A) about the y-axis showing HA still overlapping with BRP 
labeling. (D) Rotation of image (A) about the x-axis again showing HA overlapping with BRP labeling, collectively confirming that HA ramifies glomeruli. (E) Frontal section showing that HAir is absent in the AL following ablation of the cervicothoracic connective. Scale bar $=100 \mathrm{um}$. (F) Sagittal view of HA-ir in the AL following ablation between the MsG and the metathoracic ganglia in which the lesioning of metathoric HA-ir neuron axons was confirmed. Asterisks indicates MDHn cell bodies. Dashed lines indicate boundary of AL in E and F. Scale bars = 50 um.

Figure 5. Within the AL the MsHisClB receptor is expressed by a subset of GABAergic LNs and one FMRF-amidergic and one ATRergic LN. (A) MsHisClB (green) and GABA (magenta) colabeling in the lateral cell cluster of the AL. MsHisClB-ir is expressed in all AL glomeruli. Scale bar $100 \mathrm{um}$. (B) GABA-ir and MsHisClB-ir expression in the lateral cell cluster. (C), (D). Inset from (B) highlights a single large MsHisClB-ir cell body that does not express GABA. (E) FMRFamide-ir (cyan) and MsHisClB-ir (green) expression in the lateral cell cluster. (F), (G) Inset from (E) highlights a single large cell body that expresses both MsHisClB-ir and FMRFamide-ir. (H) ATR-ir (orange) and MsHisClB-ir (green) expression in the lateral cell cluster. (I), (J) Inset from $(\mathbf{H})$ highlights a single large cell body that expresses both MsHisClB-ir and FMRFamide-ir. All scale bars $=50$ um unless otherwise noted.

Figure 6. The MDHns survive metamorphosis, but the MsHisClB receptor is not expressed in the larval antennal center (LAC). (A) Horizontal view of HA-ir in the 5th instar larval MsG shows highly similar cell morphology and radiation patterns of fine processes as in the adult MsG. (B) HA-ir in the larval brain (green) shows extensive branching in the tritocerebrum (dash-dot line), but very little innervation in the LAC (dashed line). Syto-59 (magenta) highlights the boundary of the tritocerebrum and LAC. (C) MsHisClB-ir (green) is present in the tritocerebrum, but not in the LAC. LAC and tritocerebrum highlighted with Syto-59 (magenta) as in (B). All scale bars $=100$ um.

Figure 7. Schematic of the proposed MDHn circuit. (A) Manduca with overlaid nervous system cartoon. (B) Schematic of the MDHn cells from the thoracic ganglia to the AL. Only one cell is shown in detail with processes radiating in the MsG, a small process in the prothoracic ganglion, projecting up the cervicothoracic connective, a branch to the AMMC, and bilateral projections to each AL. (C) MDHn projection entering the ventral AL (green) along with the proposed AL circuitry. For the sake of simplicity, only the processes from MsHisClB-ir expressing neurons (green outline) are shown. MsHisClB-ir GABAergic (pink with green outline) and peptidergic (cyan or orange with green outline for FMRFamide and allatotropin, respectively) LNs ramify each glomerulus. Other cell types are also present including PNs (open circles), GABAergic LNs (pink circles with black outlines), ATR LNs (orange circles with black outline), and FMRF LNs (blue circles with black outline). $\mathrm{AL}=$ antennal lobe; oe=esophageal foramen; SEZ=subesophageal zone; $\mathrm{CTC}=$ cervicothoracic connective; $\mathrm{PtG}=$ prothoracic ganglia; $\mathrm{MsG}=$ mesothoracic ganglia; $\mathrm{MtG}=$ metathoracic ganglia; ab1=abdominal ganglion 1; ab2=abdominal ganglion 2 . 
Tables

Table 1. Primary antibodies used in this study

\begin{tabular}{|c|c|c|c|c|c|}
\hline Antigen & Immunogen & $\begin{array}{l}\text { Manufacturer, } \\
\text { host, monoclonal } \\
\text { vs. polyclonal }\end{array}$ & $\begin{array}{l}\text { Catalog } \\
\#\end{array}$ & RRID & $\begin{array}{l}\text { Dilution } \\
\text { used }\end{array}$ \\
\hline $\begin{array}{l}\text { Histamine } \\
\text { (HA) }\end{array}$ & $\begin{array}{l}\text { Synthetic histamine } \\
\text { coupled to } \\
\text { succinylated keyhole } \\
\text { limpet } \\
\text { hemocyanin with } \\
\text { carbodiimide linker }\end{array}$ & $\begin{array}{l}\text { Immunostar, } \\
\text { Rabbit, } \\
\text { polyclonal }\end{array}$ & 22939 & AB_572245 & $1: 500$ \\
\hline $\begin{array}{l}\text { Bruchpilot } \\
\text { (BRP) }\end{array}$ & $\begin{array}{l}\text { Bruchpilot peptide } \\
\text { sequence (1390- } \\
\text { 1740) from head } \\
\text { homogenate }\end{array}$ & $\begin{array}{l}\text { DSHB, Mouse, } \\
\text { monoclonal }\end{array}$ & Nc-82 & AB_2314866 & $1: 50$ \\
\hline $\begin{array}{l}\text { Manduca sexta } \\
\text { Histamine B } \\
\text { receptor } \\
\text { (MsHisClB) }\end{array}$ & $\begin{array}{l}\text { Histamine B } \\
\text { receptor peptide } \\
\text { sequence } \\
\text { (VNPDIELPQLD) }\end{array}$ & $\begin{array}{l}\text { Bethyl } \\
\text { Laboratory } \\
\text { (custom), Rabbit, } \\
\text { polyclonal }\end{array}$ & N/A & N/A & $1: 5000$ \\
\hline $\begin{array}{l}\gamma \text {-aminobutyric } \\
\text { acid (GABA) }\end{array}$ & $\begin{array}{l}\text { Purified GABA } \\
\text { conjugated to BSA }\end{array}$ & $\begin{array}{l}\text { Abcam, Mouse, } \\
\text { monoclonal }\end{array}$ & ab49675 & AB_880138 & $1: 500$ \\
\hline $\begin{array}{l}\text { Allatotropin } \\
\text { (ATR) }\end{array}$ & $\begin{array}{l}\text { Allatotropin coupled } \\
\text { to thyroglobulin with } \\
\text { glutaraldehyde }\end{array}$ & $\begin{array}{l}\text { Dr. Jan Veenstra, } \\
\text { Rabbit, } \\
\text { polyclonal }\end{array}$ & N/A & AB_2313973 & $1: 8^{*}$ \\
\hline $\begin{array}{l}\text { FMRF-amide } \\
\text { (FMRF) }\end{array}$ & $\begin{array}{l}\text { Synthetic FMRF- } \\
\text { Amide coupled to } \\
\text { bovine thyroglobulin } \\
\text { with gluteraldehyde }\end{array}$ & $\begin{array}{l}\text { Dr. Eve Marder, } \\
\text { Rabbit, } \\
\text { Polyclonal }\end{array}$ & N/A & AB_572232 & $1: 8^{*}$ \\
\hline
\end{tabular}

* See fluorescent tagging subsection of the methods for details. 


\section{Figure 1:}

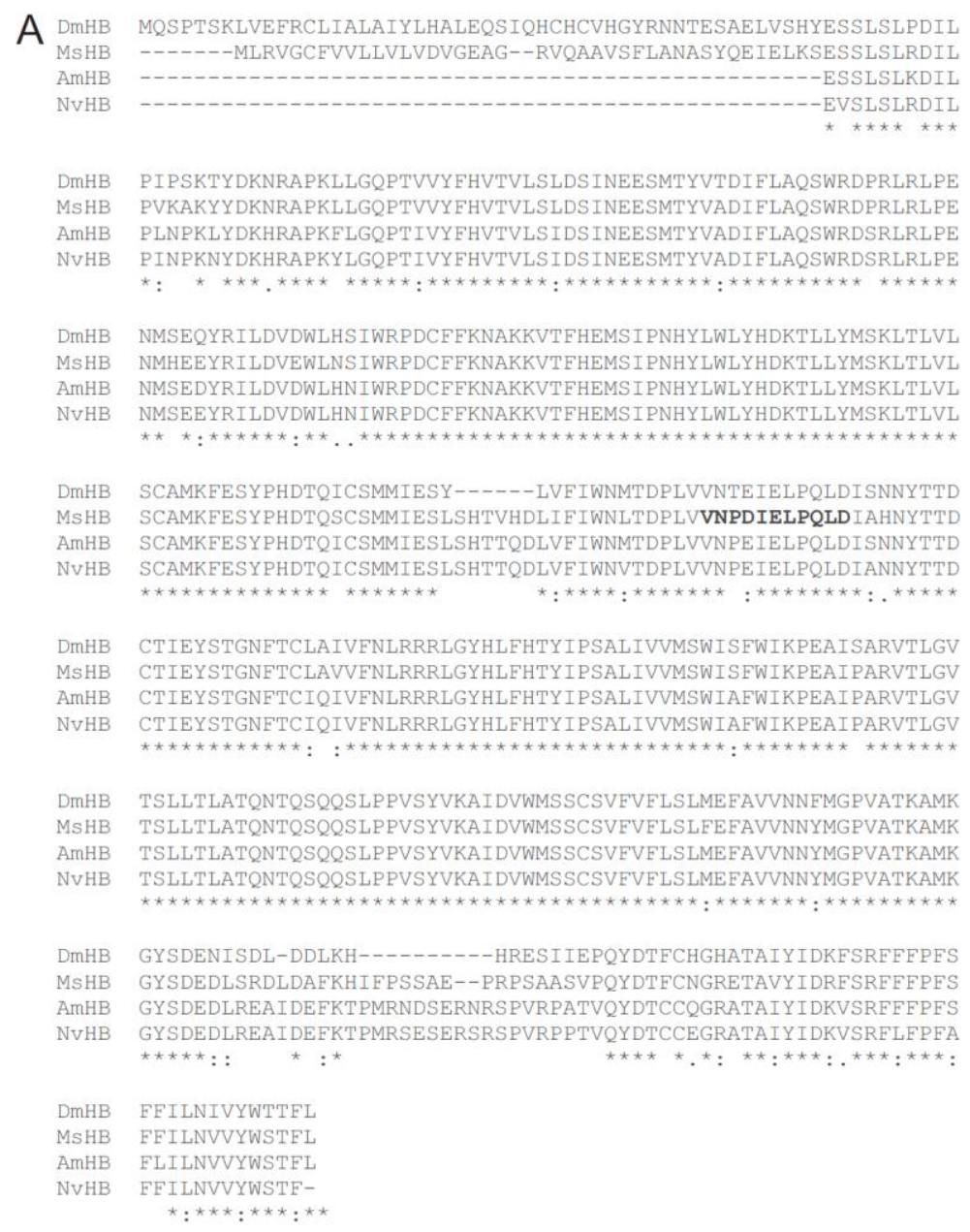

B
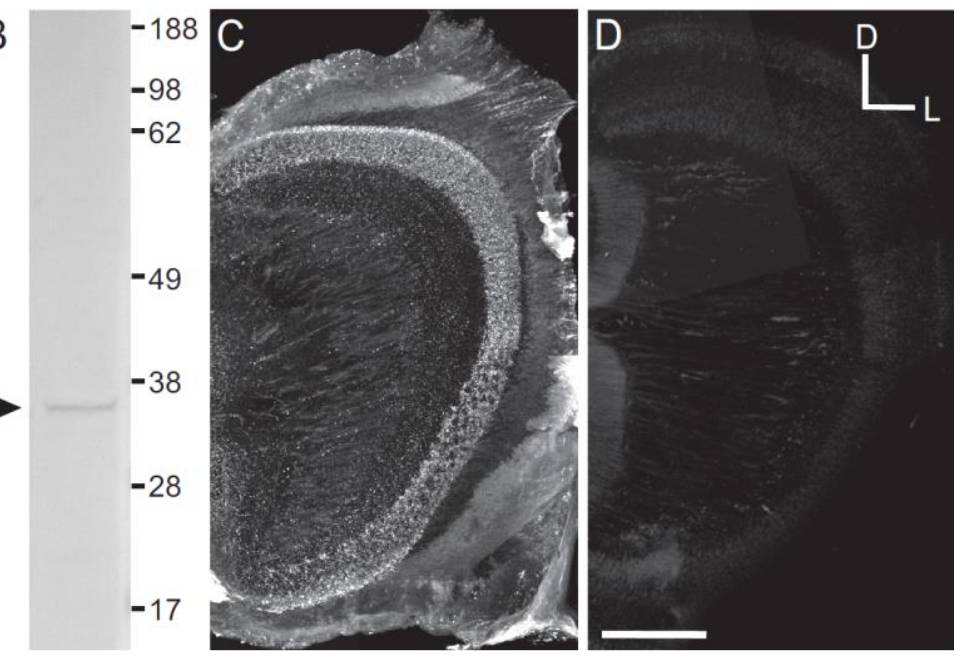
Figure 2:

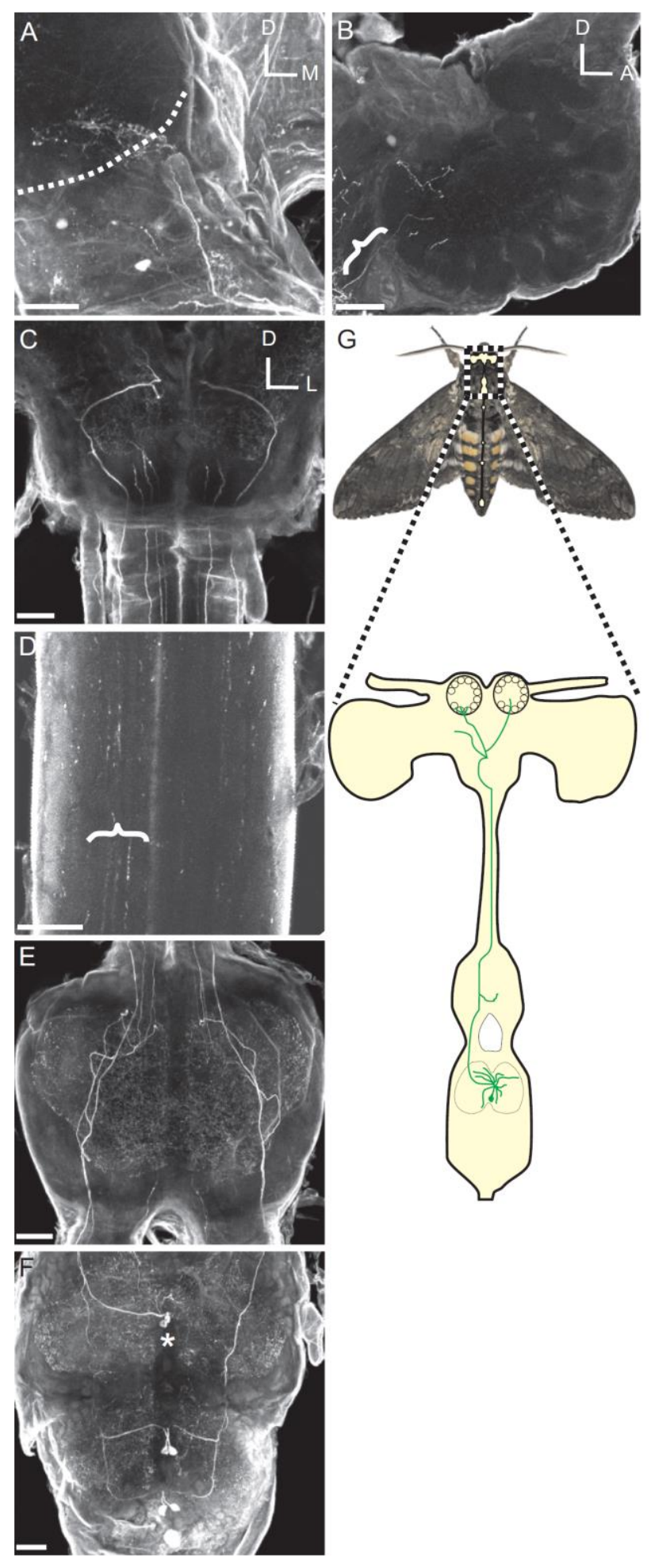


Figure 3:
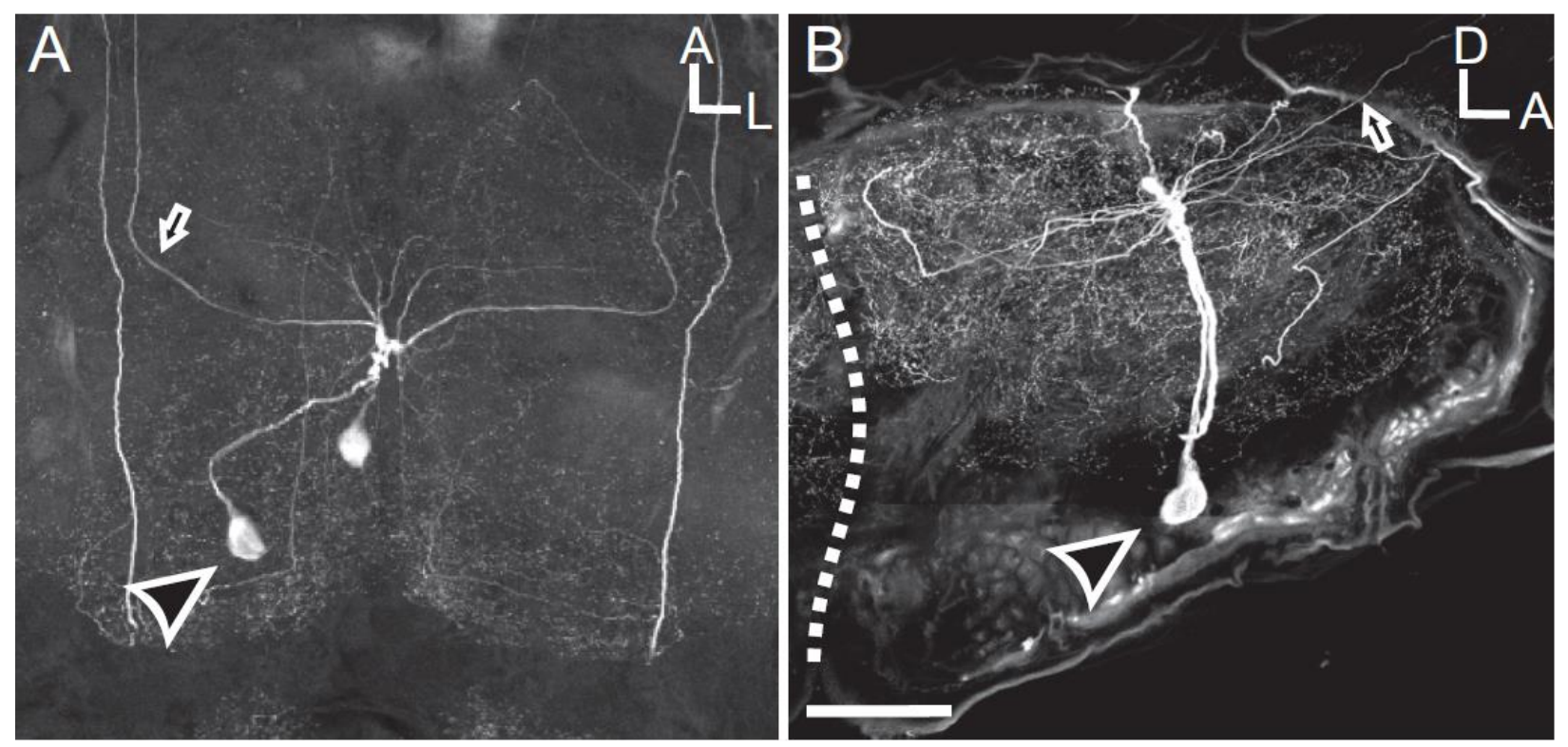
Figure 4:

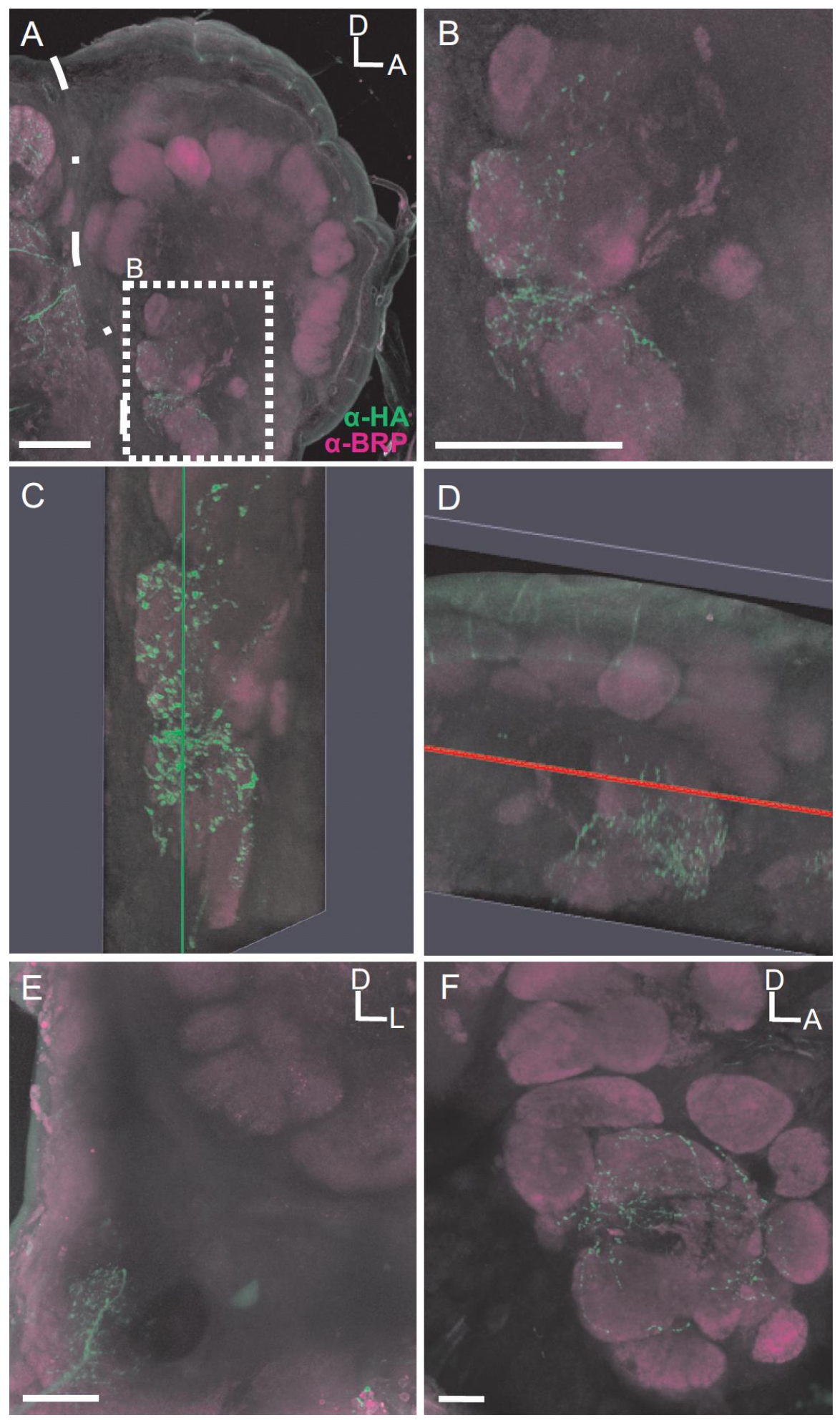


Figure 5:

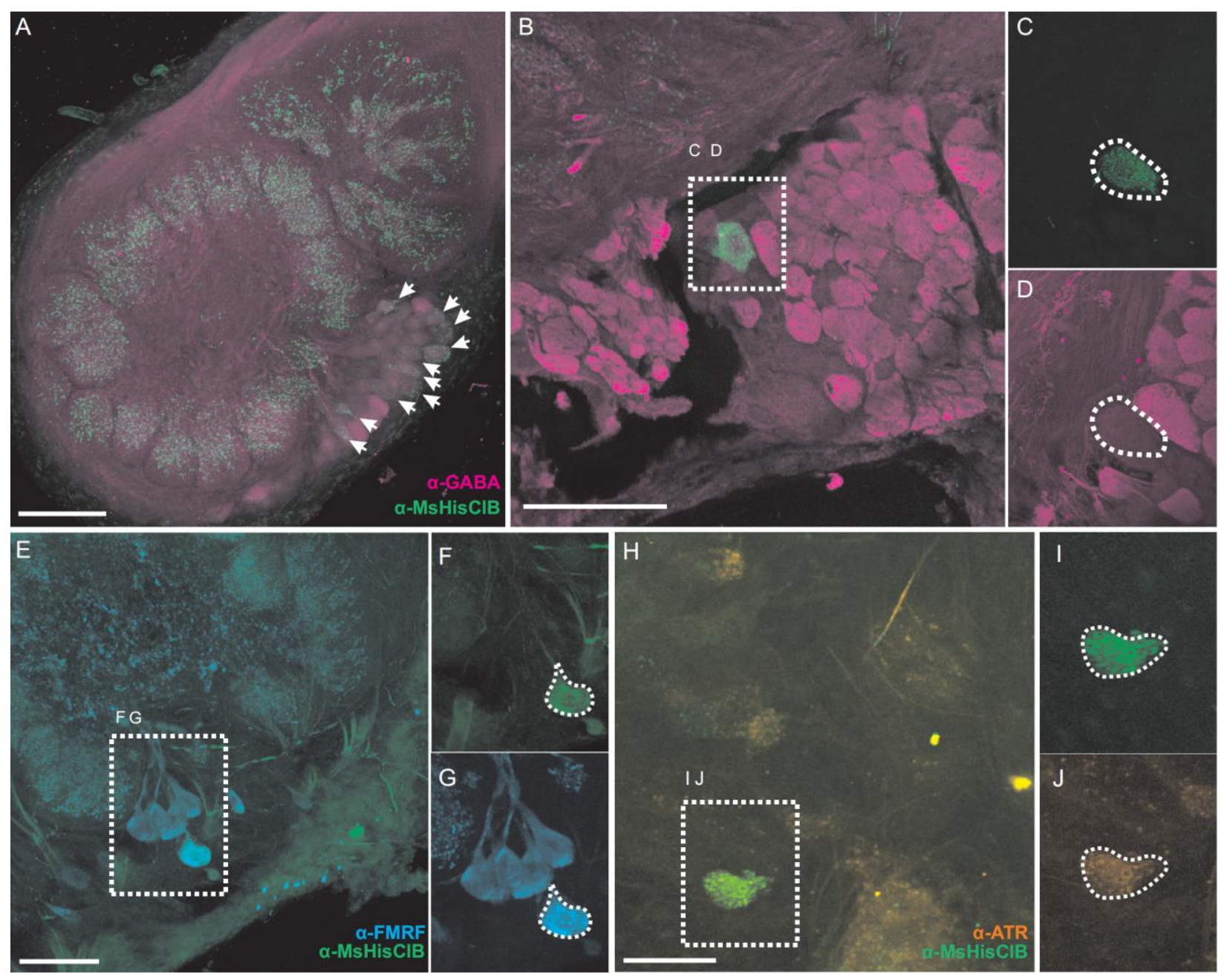


Figure 6:
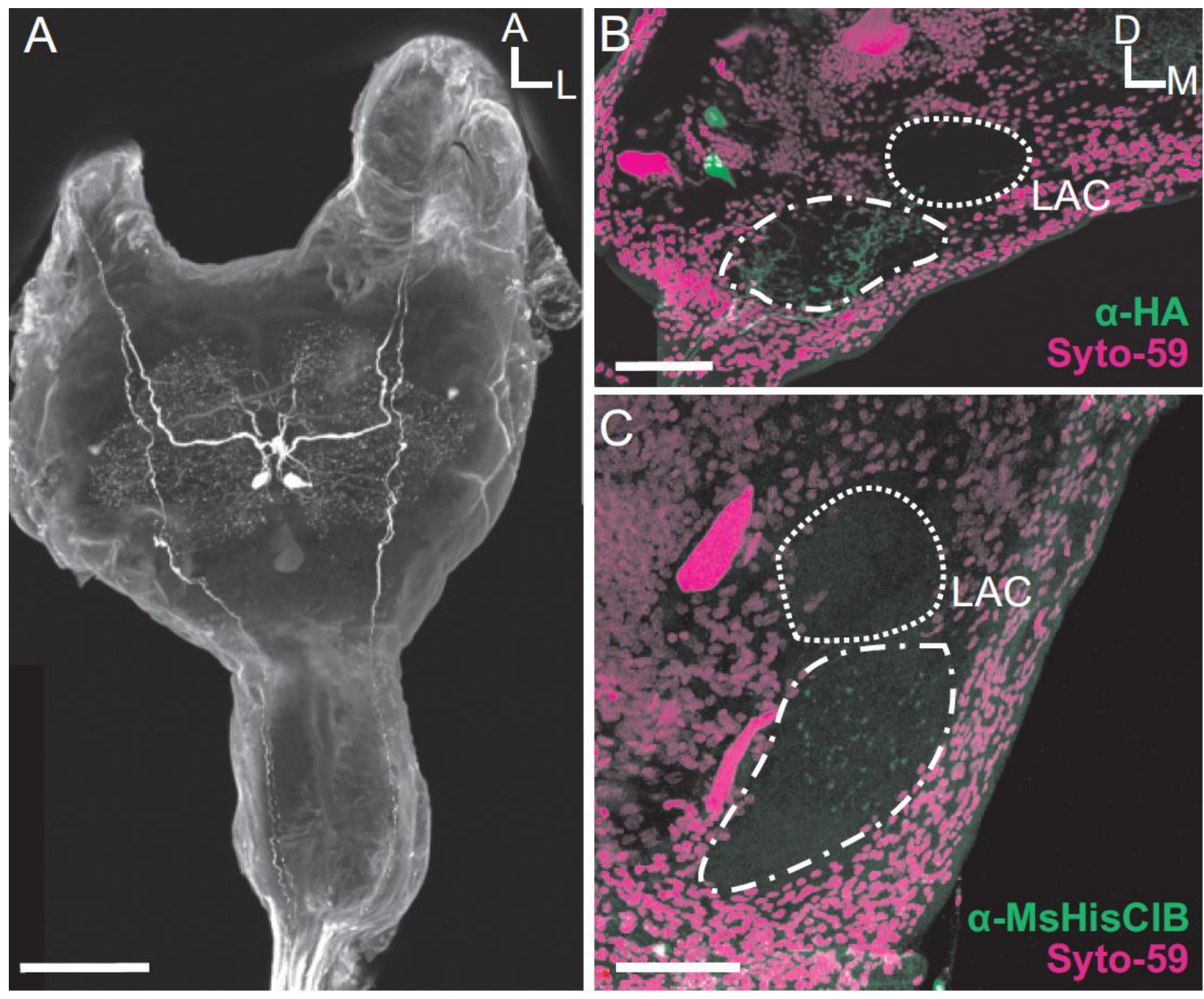
Figure 7:

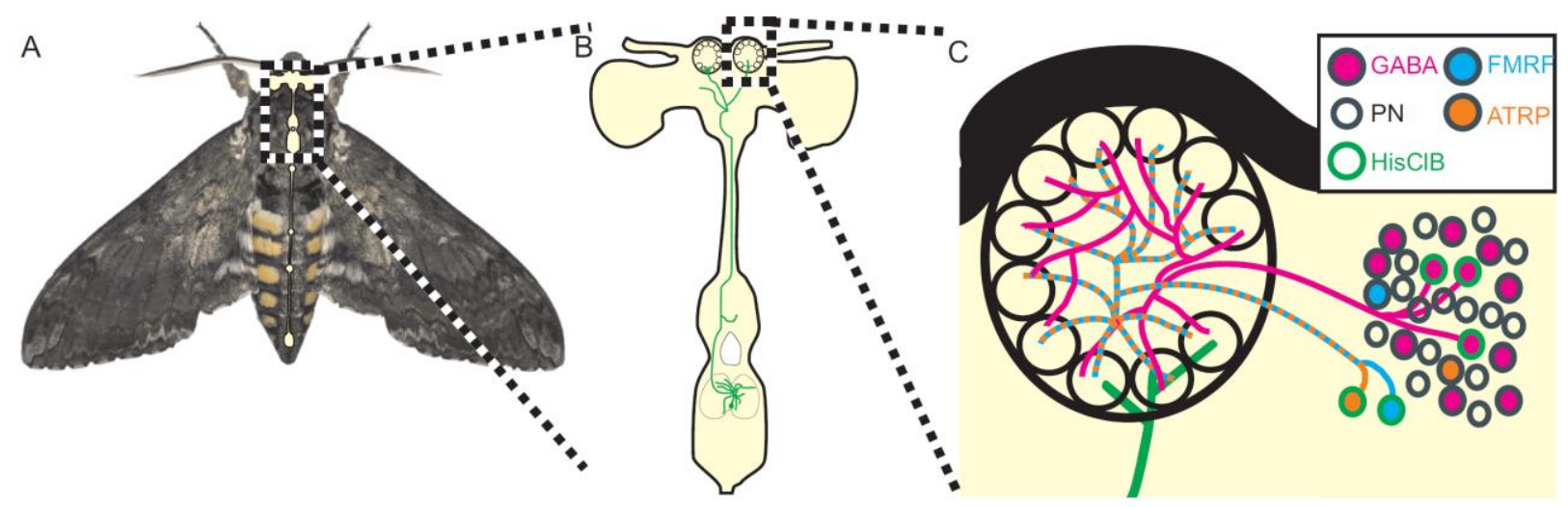




\section{Appendix B:}

\section{PNAS Classification: Biological sciences, Neuroscience}

Title: Flight Motor Networks Modulate Primary Olfactory Processing: A Novel Corollary Discharge Circuit in the Moth Manduca sexta.

Phillip D. Chapman ${ }^{1}$, Rex Burkland ${ }^{1}$, Samual P. Bradley, Benjamin Houot ${ }^{2}$, Victoria Bullman, Andrew M. Dacks, Kevin C. Daly

West Virginia University, Morgantown, WV, United States

${ }^{1}$ These authors contributed equally to this work.

${ }^{2}$ Division of Chemical Ecology, Department of Plant Protection Biology Swedish University of Agricultural Sciences, Box 102, S-230 53 Alnarp Sweden

\section{Abstract:}

Nervous systems must distinguish sensory signals derived from an animal's own movements (reafference) from environmentally derived sources (exafference). To accomplish this, motor networks producing reafference transmit motor information, via a corollary discharge circuit (CDC), to affected sensory networks, modulating sensory function during behavior. While CDCs have been described in most sensory modalities, none have been observed projecting to an olfactory pathway. In moths, two histaminergic neurons (MDHns) project from flight sensorimotor centers in the mesothoracic neuromere to the antennal lobe $(A L)$ where they provide the sole source of histamine (HA), but whether they serve a CDC function is unknown. We demonstrate that MDHn spiking activity is positively correlated with wing motor output and increased prior to bouts of motor activity, suggesting that MDHns communicate global locomotor state (consistent with a "higher-order" CDC), rather than providing a precisely timed motor copy. Within the AL, HA application sharpened entrainment of $P N$ responses to odor stimuli embedded within simulated wing beat induced flows, whereas MDHn axotomy or AL HA receptor (HA-r) blockade reduced entrainment. This finding is also consistent with higher order CDCs, as the MDHns enhanced rather than filtered entrainment of AL PNs. Finally, HA-r blockade increased odor detection and discrimination thresholds in behavior assays. These results establish the MDHns as a higher order CDC that modulates AL temporal resolution, enhancing odor-guided behavior. The MDHns thus appear to represent a novel higher order CDC to the olfactory pathway of an insect, the unique nature of this CDC highlights the importance of motor-to-sensory signaling as a context-specific mechanism to finetune sensory function.

Acknowledgements: We thank Mark Willis for fruitful discussions during the course of this research and Sadie Bergeron and Gary Marsat for comments on this manuscript. This research was supported by NIH DC009417 to KCD and AFOSR FA9550-17-1-0117 to KCD and AMD.

Significance: Across vertebrates and invertebrates, corollary discharge circuits (CDCs) project to and inform sensory networks about an animals' movements, which directly impact sensory 
processing. Failure of CDCs likely underlie sensory hallucinations in schizophrenia, Parkinson's disease and dyspnea, highlighting the fundamental importance of CDCs for successfully interpreting sensory cues to adaptively interact with the external world. Ultimately, understanding the role of CDCs in integrating sensory motor function will be vital to understand these diseases, but mechanistically little is known about how CDCs function. CDCs have been identified in most sensory domains except olfaction. Our findings indicate that a histaminergic CDC enhances the ability of the olfactory system to more precisely encode stimulus temporal structure, resulting in enhanced olfactory acuity.

\section{Introduction:}

As animals locomote, their motor actions can directly affect sensory function, causing selfinduced, or "reafferent", sensory neural responses. Unchecked, reafference can interfere with or otherwise influence the experience of externally derived or "exafferent", sensory cues. Furthermore, behaviors causing reafference can be an integral component of active sensory sampling strategies. For instance, saccadic eye movements continually shift the retinal image in a ballistic fashion to interrogate the visual environment and yet visual experience is perceived as stable. This visual stabilization likely occurs because the superior colliculus sends information about eye movement commands to the frontal eye field of the cortex[1]. Such motorto-sensory pathways are referred to as corollary discharge circuits (CDCs), which are a class of forward circuits that specifically provide information about motor activity to sensory systems, allowing them to account for behavior-induced effects on sensory function. CDCs can provide precisely timed facsimiles of motor commands (i.e., an efference copy) to modulatory-like signals that represent current or pending changes in behavioral state [2]. While all CDCs provide motor information to sensory systems, they can be further classified based on their functional consequences on sensory processing. CDCs that filter out reafferent inputs or inhibit sensory-driven reflexes [e.g. 3, 4] are classified as lower order CDCs, while tho se that predict, stabilize, facilitate sensory signal analysis, or sensory motor learning [e.g. 5, 6] can be classified as higher order CDCs[2]. Given their fundamental role in sensory-motor interactions, evidence of CDCs have been observed in vision[2, 5-7], hearing [4, 8, 9] and the sensing of body posture[10, 11], and their failure likely underlies sensory hallucinations in schizophrenia[12], Parkinson's disease[13] and dyspnea[14]. Indeed, CDCs have been characterized to some degree in nearly every sensory domain except olfaction, and to date no higher order CDC has been described in any invertebrate nervous system.

Like eye saccades in vertebrates, active olfactory sampling behaviors such as sniffing, antennal and tongue flicking, and wing beating are periodic[15]. These active sampling behaviors increase air flow and turbulence around the olfactory epithelium, inducing a mechanosensory component to olfactory neural responses observable even in the absence of odor[16-19]. In the hawkmoth Manduca sexta and other related insects, wing beating, in addition to casting back and forth through odor plumes, are an important component of active odor sampling behavior[20-22]. Wing beating can generate substantial oscillatory airflow over the antennae[23] and vibrates the antennae at the wing beat frequency during flight[24]. This implies that during odor-guided flight, olfactory sensory neurons on the antennae are periodically exposed to odorant molecules in higher velocity flows induced by wing beating, presumably enhancing odor-receptor interactions[21]. Far from hindering moths, periodic odor stimulation is readily tracked by both local interneurons (LNs) and projection neurons (PNs) of the antennal lobe (AL; the primary olfactory network)[25]. Pulsed delivery of odors elicits more 
distinctive AL odor representations relative to continuous odor stimulation[18], and appears to be required for several moth species to track and locate odor sources[20, 26, 27]. In theory, the ability to track odors presented at the wing beat frequency could arise from purely feedforward mechanisms[28]. However, AL neural tracking of stimuli presented at wing beat frequencies requires neural connectivity between flight motor circuits in the thoracic neuromeres and the $\mathrm{AL}[25]$, suggesting that motor centers may directly influence the temporal resolution of the olfactory system. The only known connection between the flight motor pattern generating centers and the olfactory system in $M$. sexta is a single pair of mesothoracic to deutocerebral histaminergic (HA) neurons (MDHns); these cells represent the exclusive source of HA in the $\mathrm{AL}[29,30]$. Within each $\mathrm{AL}$ of $M$. sexta, 16 predominantly GABAergic LNs express the HA-B receptor $(\mathrm{MsHisClBr}$ ) and collectively these $L N$ s ramify all $A L$ glomeruli whereas the $H A-A$ receptor was not observed[30]. In arthropods, there are only two known HA receptors, both of which are inhibitory ionotropic $\mathrm{Cl}^{-}$channels[31-33], suggesting that MDHns disinhibit the $\mathrm{AL}$ network when active.

Adult $M$. sexta primarily fly to locomote and use their legs to grasp objects that they land upon, this suggests that MDHn function primarily relates to flight behavior. Consistent with this, the MsHisClBr is not expressed within the larval antennal center[30], despite the MDHns being present and projecting to these centers across all larval stages. This implies that that this circuit only becomes functional in adults and takes on a flight related role. In most insects, the MDHns project to the sub esophageal zone and antennal mechanosensory and motor center (AMMC). However, in nocturnally active plume tracking insects like caddisflies[34] and moths, the MDHns innervate the AL as well[35]. Interestingly, this circuit appears to have been lost in closely related butterflies[35], which are diurnal and differ from moths in their flight mechanics and relative reliance on visual, rather than olfactory cues. The MDHns are therefore excellent candidates for a CDC between flight sensory motor centers in the ventral nerve cord and the olfactory system in the moth brain, however neither their function during flight nor their functional role in olfactory processing and odor-guided behavior is known.

Here we demonstrate that the MDHns function as a higher order, flight-to-olfactory CDC. We show that MDHn spiking activity is positively correlated to the ongoing level of wing motor output and increased MDHn spike rate precedes bouts of motor output. Furthermore, increasing AL HA enhances entrainment to olfactory stimuli presented at the wing-beat frequency, while disrupting AL HA-r function or removing MDHn input reduces entrainment. Finally, disruption of AL HA-r function decreases olfactory acuity in behavioral detection and discrimination threshold assays. Collectively, these results lead to the conclusion that during flight, the MDHns which likely disinhibit the $A L$ network, upregulate $A L$ entrainment to the stimulus temporal structure thereby enhancing olfactory acuity in behavioral assays. MDHns therefore do not filter the effects of wing beat induced sensory reafference from the neural response as would be the case in a lower order CDC. Rather, the MDHns upregulate the ability of the olfactory system to entrain to the temporal features of the odor stimulus and enhance the ability of moths to both detect and discriminate between odors. While several studies in insects have characterized different neural circuit mechanisms that coordinate modulation of sensory processing with changes in behavioral state[8, 36], our results appear to represent a novel higher order motor-to-olfactory CDC. 


\section{Results:}

MDHn activity is positively correlated with forewing motor output. The MDHns arborize throughout the dorsal aspect of the mesothoracic neuromere, which along with the metathoracic neuromere, house flight central pattern generating circuitry, including wing sensory input[3739]. To characterize the relationship between the MDHns and wing motor output, we developed an approach that leaves the entire central nervous system intact, exposing the mesothoracic neuromere where all sensory motor nerves emanating from the thoracic neuromeres were sectioned for stability; this also allowed us to make intracellular recordings from individual MDHns while simultaneously recording forewing depressor and elevator motor neuron output from the trunk IIN1b fiber using a suction electrode[40] (Fig. 1A). Intracellular electrode guidance to MDHns was visually blind, but spike shape and a tonic firing pattern guided selection of specific cells for recording. Each recorded cell was dye filled, and HAimmunolabeling was used to confirm that an MDHn was recorded (Fig 1B).

All recorded $\mathrm{MDHns}(\mathrm{N}=5)$ produced highly stereotyped spike waveforms and were tonically active even in the absence of motor output (Fig 1C). In 4 of the 5 animals we were able to the hold intracellular recording long enough to induce wing motor output via bath application of chlordimeform $\left(10^{-5} \mathrm{M}\right)$ an effective and selective octopamine agonist known to reliably induce fictive flight in insects, including $M$. sexta[37]. In all cases MDHn tonic spike frequency was positively correlated with the presence and strength of wing motor output (Fig. 1C-G). This correlation could indicate that the MDHns receive input from motor circuitry or that chlordimeform directly affects the MDHns in parallel with motor circuitry. However, increases in MDHn firing rate were coupled to individual brief bouts of wing motor output (Fig. 1C), suggesting that MDHn activity was coupled to motor output per se and not necessarily chlordimeform application. This also suggests that MDHns were driven by network components that produce and regulate the initiation and cessation of wing motor output. In cases where wing motor output increased or otherwise remained tonically active on a time scale of minutes, MDH activity increased in coordination with gradual increases in motor output (Fig. 1D). Mean normalized spike rate of both MDHn and IIN1b were positively correlated across all recordings (Fig. 1E) and manually segmenting recordings into epochs of wing motor output versus "quiescence" (SI Methods) demonstrated a significant increase in MDHn spike rate during wing motor output (Fig. 1F). Thus, the activity of MDHns represents a corollary of wing motor output.

MDHn activity could provide two types of information about wing motor output. MDHn spiking activity could be a precise efference copy of wing motor function (indicative of a lowerorder $\mathrm{CDC}$ ), or rather than encoding precise wing movement, MDHn spiking activity could reflect the current behavioral state of the flight motor network (observed in higher order CDCs). Cross-correlation analysis revealed no temporally precise spiking relationships between the recorded motor output fiber and MDHn (Fig. 1G). Rather, MDHn activity preceded bouts of motor activity by approximately $100 \mathrm{~ms}$ and the correlation between MDHn and IIN1b spiking was only evident when data was smoothed across $25 \mathrm{~ms}$ or more (Fig. 1G), indicating that MDHn spiking activity and flight motor output were correlated on a slower timescale. Thus, while MDHn and wing motor output appear to be driven by an at least partially overlapping circuitry, the MDHns do not represent a precise efference copy per se. This is further supported by the observation that in all MDHn recordings, there was persistent tonic spiking in the absence of motor output. Thus, MDHns appear to encode changes in behavioral state. 


\section{Antennal lobe neural entrainment to stimulus temporal structure is modulated by}

histamine. MHDns increase their spiking activity during wing motor output, thus it stands to reason that $\mathrm{HA}$ release in the $\mathrm{AL}$ increases as well. We next asked whether $\mathrm{HA}$ release from the MDHns, which are the exclusive source of HA in the AL of M. sexta[30], affects neural responses to temporally patterned odor stimulation that simulates the periodic flow effects induced by wing beating[23]. We therefore used extracellular tetrodes to record simultaneously from multiple individual AL neural units[41] while stimulating the antenna with a single odorant (either 2-hexanone or 2-octanone). Odor was presented in blocks of five $500 \mathrm{~ms}$ long $20 \mathrm{~Hz}$ pulse trains using a $50 \%$ duty cycle (i.e. $25 \mathrm{~ms}$ on and $25 \mathrm{~ms}$ off) and $10 \mathrm{sec}$ between each train of a block. This was repeated every two minutes for $30 \mathrm{~min}$. After the first block, the moth received one of the three treatments. On average 18-22\% of AL neural units within each group entrained to odor pulse trains (Fig. 2A). Based on their spiking characteristics, these units can be putatively classified as PNs[42]. First, to disrupt HA function, we bath applied the HA-r antagonist cimetidine ( $500 \mu \mathrm{M}$; Fig. $\left.2 \mathrm{~A}_{\mathrm{i}}\right)$. In many units that were initially able to entrain to $20 \mathrm{~Hz}$ pulsed stimuli, cimetidine application decreased their ability to entrain to stimulus temporal structure. For example, the unit depicted in Figure $2 \mathrm{~A}_{\mathrm{ii}}$, initially responded reliably to all ten pulses of the pulse train across all 5 repeats as indicated by 10 prominent peaks in the inset histogram. After cimetidine application, the same unit failed to reliably entrain to the stimulus; it failed to respond to the first two pulses then consistently responded to three, perhaps 4 subsequent pulses. To evaluate the ability of units to entrain to the $20 \mathrm{~Hz}$ pulse trains, we calculated power spectral densities for each unit in response to each stimulus block, then calculated the integrated power from 18-22 Hz[25]. Cimetidine application significantly decreased the mean integrated power across units over time indicating that, relative to time matched controls, the ability of units to entrain to pulsed odor had degraded within 6 minutes of application (Fig. 2A $\mathrm{A}_{\text {iii }}$ ). If blocking HA-r function reduces the ability of $\mathrm{AL}$ neurons to entrain to pulsed stimuli, it stands to reason that removing input from the sole source of HA in the AL (the MDHns[30]) should have the same effect. Therefore, our second approach was to sever the neck connective in a second group of moths, thus axotomizing the two MDHn axons therein (Fig. $2 \mathrm{~B}_{\mathrm{i}}$ ). As with cimetidine application, we observed that in moths where the neck connectives were cut, units that were initially able to reliably track pulsed odor were less able to track over time relative to time-matched sham surgery controls (e.g. Fig. $2 \mathrm{~B}_{\mathrm{ii}}$ ). Across the population this manifest as a significant reduction in integrated power around the pulsing frequency within 16 min (Fig. 2B $\mathrm{B}_{\mathrm{iii}}$ ). It is important to note that entrainment across the population was not completely lost in either case. Rather, there was a loss of responses to individual pulses of a train (Fig. 2A inset red box) and/or the relative degradation in ability of the cell to produce discrete bursts to individual pulses separated by interstitials with no spiking (see Fig. 2Bii, before vs after). Finally, if disrupting the MDH circuit degrades the temporal fidelity of odor encoding, bath application of HA should have the opposite effect. Therefore, in a final group of moths HA (50 $\mu \mathrm{M})$ was bath applied during pulsed odor stimulation (Fig. 2C $\mathrm{C}_{\mathrm{i}}$ ). Within 4 minutes of initiating HA application, the ability of individual units within the ensemble to entrain to the stimulus temporal structure increased and in some instances, units that did not initially entrain to odor pulses were recruited into the population of entrained units (e.g. Fig. $2 \mathrm{C}_{\text {ii) }}$. Across the population, we observed a significant increase in mean integrated power at the pulsing frequency relative to controls (Fig. $\left.2 \mathrm{C}_{\mathrm{ii}}\right)$. This HA-induced increase in power only occurs at the pulse frequency and does so as the overall population spiking response to the pulse trains increases as well (SI Fig. 1). These results collectively indicate that $\mathrm{MDHn}$ release of $\mathrm{HA}$ within the $\mathrm{AL}$ enhances entrainment to the 
stimulus temporal structure as opposed to filtering out the reafference. This again supports the notion that this circuit be classified as a higher order CDC[2].

Olfactory acuity is histamine dependent. The capacity for the olfactory system to guide behavior is fundamentally dependent on its ability to detect and identify (i.e. discriminate) important odors. Simulating wing beat induced flows enhances both separation of neural representations of different odors[18] and olfactory acuity in behavioral assays[25, 43].

Therefore, we predict that because this CDC enhances odor processing it will likewise enhance behavioral measures of odor detection and discrimination. Two behavioral assays were used to determine if HA circuit function contributes to the detection and discrimination of odors (see SI Methods and Materials). Both assays were based on a well-established Pavlovian olfactory learning approach[44-49] where moths were first conditioned (for detection assays) or differentially conditioned (for discrimination assays) to respond to target odors. Twenty-four hours post conditioning, moths were randomly assigned into drug or control injection treatments and tested in a blind format. Based on initial control experiments (See SI Fig. 2), an effective dose of $\sim 1 \mathrm{nl}$ of $50 \mu \mathrm{M}$ cimetidine was injected into each AL. Fifteen minutes following injection, moths were challenged with a blank stimulus then a series of increasing concentrations of the conditioned odor to determine the concentration at which they detected the odor as measured by a significant increase in conditioned feeding response relative to the response to blanks. Both groups acquired the conditioned response (Fig 3A). However, tests subsequent to injection indicated that cimetidine injected moths displayed an order of magnitude higher detection threshold relative to controls (Fig. 3B). This was replicated using a different HA-r antagonist, ranitidine (see SI Materials and Methods, SI Results and SI Fig. 3). Collectively, these results suggest that HA within the AL enhances olfactory sensitivity.

As detection is a prerequisite for identification[45, 48], it stands to reason that increased detection thresholds would also impact the moths ability to identify target odors. Here we observed that discrimination thresholds also increased when HA-r function was blocked. Moths in both drug treated, and control groups learned to differentially respond to the CS+ and CSodors (Fig. 3C). Again, 24h after conditioning, moths were injected with either cimetidine or the saline vehicle, this time in a double-blind format. We then tested with both the CS+ and CSodors across a dilution series of increasing concentration to determine the odor discrimination threshold, the lowest odor concentration at which moths responded significantly more to the CS+ relative to the CS- (i.e. a "conditioned differential response"; see SI methods). The discrimination threshold for saline injected moths occurred at an odor concentration of 0.1 $\mu \mathrm{g} / 2 \mu \mathrm{l}$, but when injected with cimetidine, a significant differential response was observed at $10.0 \mu \mathrm{g} / 2 \mu \mathrm{l}$. Thus, disruption of HA-r function decreases both the ability to detect (Fig. 3B) and identify (Fig. 3D) odors.

\section{Discussion:}

Nervous systems must coordinate sensory with motor network function to adjust sensory processing based on planned and ongoing motor activities. CDCs are one class of neural circuits that provide information about motor output to sensory pathways to optimize sensory processing within the context of specific behaviors. CDCs can be broadly classified into two categories, "lower-order" and "higher-order", defined based on the functional consequence they have on their target sensory pathway[2]. Lower-order CDCs directly inhibit the reafference with 
precisely timed spikes that gate sensory signals[4]. Higher-order CDCs on the other hand, can activate hundreds of milliseconds prior to the onset of a behavior and can modulate the state of a sensory network to accommodate imminent changes in behavior[9]. Furthermore, higher order CDCs do not block or filter the reafferent sensory input, rather they exploit the reafferent input to facilitate sensory processing[2]. Our results indicate that the MDHns are a higher-order CDC that functions to disinhibit the $A L$ in advance of imminent motor actions of the wings, enhancing the ability of the AL to entrain to the stimulus temporal structure. MDHn firing rate increases just prior to and during wing motor output, but is not synchronous with IIN1b motor neuron spiking, suggesting that the MDHns do not provide precise information about the timing of motor output (i.e. an efference copy), but rather they appear to represent the broad behavioral state of flight.

The input signals that drive MDHn activity remain unknown though the list of candidates is relatively small and includes sensory afferents from the wings, legs and thorax; central neurons that mediate motor patterns; and the motor neurons themselves. Sensory afferents are unlikely to drive MDHns as our approach was to cut all thoracic sensory afferent (and motor) fibers; this occurred $\sim 45$ min prior to recording. Furthermore, in $M$. sexta, MDHn local processes within the mesothoracic neuromere are restricted to its dorsomedial aspect[30], while sensory afferents in a closely related moth species predominately innervate its ventrolateral aspect[39]. However, we cannot rule out the possibility that sensory input to the pterothoracic ganglia normally contributes to MDHn activity in intact animals. Additionally, MDHn activity precedes wing motor output, making it unlikely that motor output drives their activity either. Thus, our anatomical and physiological data suggest that these cells are centrally (as opposed to peripherally) driven.

The ability of the olfactory system to track odor timing is highly dependent on LNs that control a variety of network-wide coding features including the transient nature of PN responses[50, 51]. LNs therefore represent an elegant target for CDCs to regulate a sensory network. Pulse tracking is only weakly present in antennal field recordings in M. sexta but dominates AL local field potentials and spiking in at least some PNs. Furthermore, pulse tracking is both odor- and GABA- dependent, which implies lateral interactions clarify this periodic signal[25]. Thus, while GABA mediates pulse tracking in PNs, our current results suggest that the MDHns modulate this ability, and that LNs are the most likely target. Indeed, arthropods express just two HA-rs, both of which are ionotropic $\mathrm{Cl}^{-}$channels[31-33] and the $\mathrm{AL}$ of $M$. sexta, the MsHisClB receptor is expressed exclusively by 16 GABAergic AL LNs which broadly ramify the entire $A L[30]$. This implies that during flight, increased MDHn activity inhibits this subpopulation of inhibitory LNs. While the postsynaptic targets of these $16 \mathrm{LNs}$ are unknown, the consequence of HA signaling is enhancement of the AL network to encode the temporal structure of olfactory stimuli at the level of PN output. This in turn enhances detection and identification at the level of sensory perception. Given that mammalian sniffing behavior produces the same physical flow effects as wing beating, it stands to reason that an analogous system might facilitate olfaction in mammals.

If the MDHns sharpen AL entrainment to pulsed stimuli, how might this result in enhanced behavioral performance in the psychophysical assays of olfactory sensitivity and acuity? Primary olfactory networks are spontaneously active and noisy. Superimposed upon olfactory network dynamics are weak mechanosensory-driven oscillatory dynamics produced by active sampling behaviors like sniffing [16, 17] and wing beating[23, 24]. While AL neurons can be entrained to pulses of clean air[18,25], moths do not respond to these clean air pulse trains in behavioral assays (relative to the same duration continuous clean air stimulus), and thus 
oscillating mechanosensory responses from the AL are behaviorally subthreshold. However, pulsed odor stimuli are more easily detected in behavioral detection threshold assays than continuous stimuli[25, 43], suggesting that antennal and AL mechanosensory responses which are time-coupled and summate with odor-evoked activity, may facilitate stronger odor responses. Our results suggest that the MDHns fine tune AL entrainment to oscillating airflow while the moth is in flight and actively seeking odor sources, rather than canceling out these weak mechanosensory oscillations as would be the case for a lower-order CDC.

Taken together, we demonstrate that the MDHns represent an olfactory CDC that enhances olfactory processing presumably during flight. The MDHns interconnect flight motor-centers and the olfactory system, are active during wing motor output which results in enhanced the temporal fidelity of AL neurons and odor-guided behavior of moths. Thus, the MDHns meet the criteria of a CDC. Furthermore, the MDHns appear to function as a "higher-order" CDC to the $\mathrm{AL}$ as their activity sharpens temporal entrainment to the stimulus. Thus, the MDHns likely influence the ability of the AL network to track odor timing and facilitate assembly of a salient "olfactory image". Given that odor-guided behavior in M. sexta is performed primarily during flight and the MDHns originate in a flight sensory and motor pattern generating center, we propose that the MDHns optimize olfactory function within the context of odor-guided flight. Finally, given their ubiquity across insects[35] and their projections into multiple additional sensory processing centers, we have only begun to understand the multimodal nature of MDHns role in coordinating wing motor actions with sensory processing.

\section{Materials and Methods}

SI Materials and Methods detail all experimental procedures. Briefly, intracellular recordings of MDHns were made in "CNS intact" preparations that exposes the pterothoracic ganglion and lesions only nerves emanating from the pro, meso and metathoracic neuromeres to eliminate muscle contraction near the recording site. Multiunit studies of AL neural spiking responses to pulsatile stimuli were performed using a fully intact preparation described in[49]. Putative PNs are identified on spiking characteristics[42]. Equal ratios of males and females were used for all behavior pharmacology experiments. All behavioral pharmacological methods and psychophysical assays have been previously detailed[45, 47, 48].

\section{SI Materials and Methods}

\section{Subjects}

For all experiments, male and female $M$. sexta were reared in-house using documented methods[44]. At pupal stage 17, individuals were isolated in brown paper bags and placed in an incubator with a $16 / 8$ reverse light/dark cycle, at $25^{\circ} \mathrm{C}$, and $75 \% \mathrm{RH}$. Bags were checked daily; those with newly eclosed adult moths were dated. All subjects were kept in the incubator $\sim 5-7$ days post-eclosion before use.

\section{Stimulus Delivery System}

A custom built olfactometer and exhaust system was used, which has been previously described and characterized[43]. Air was provided from a centralized supply line, passed through a 500cc Drierite cartridge (Indicating Drierite, mesh 8; Drierite: 23025) then purified using a charcoal filter made from a 500cc Drierite cartridge filled with granular 20-60 mesh activated charcoal (Sigma-Aldrich: C3014). Airflow was controlled using a 150-mm direct 
reading flowmeter with an aluminum/sapphire float (Cole-Parmer: 1-010293). Filtered air was then passed through a 3-way valve (The Lee Co., LFAA1200118H). In behavioral experiments this valve was controlled by a programmable logic chip (PLC; Direct Logic, DL-05). For AL multiunit experiments, valves were controlled via opto-isolated TTL signals from the data acquisition computer and software (below). In either case, filtered air normally enters the valve then passes out the normally open exit port. Upon activation, air is shunted to a second normally closed output port that directs the airflow to an odor cartridge made from borosilicate glass attached to luer fittings with a $\sim 1.7 \mathrm{ml}$ internal volume. Odors were applied to small piece of Whatman No. 1 filter paper and inserted into the cartridge. During olfactory conditioning and for the initial dose response studies, the odor cartridge was place approximately $10 \mathrm{~cm}$ from the moth to create a more distributed plume from the olfactometer. In this case, output from the olfactometer to the animal only occurred when the valve was actuated and air passed through the cartridge. For all other experiments, both the normally open and normally closed lines were then tied into two arms of a T-fitting, which provided a constant stream of airflow over the antenna via the third arm of the T-fitting; this approach reduces the overall flow dynamics caused by valve actuation into an amplitude range consistent with what is driven by wing beating[23, 43$]$. In this case the third arm of the T-fitting was positioned $2-3 \mathrm{~mm}$ from the antenna. Effluent from the T-valve was removed by an exhaust port positioned behind the animal on the downwind side of the odor delivery stage. Each exhaust port was unique to the constraints of the staging for specific experiments but all produced an ambient airflow of $0.3 \mathrm{~m} / \mathrm{s}$ at the position of the antenna and was calibrated using a hotwire anemometer (Fisher Scientific).

\section{Dual intracellular/extracellular recordings}

To characterize the relationship between spiking of the MDHns and motor output to the wing muscles we made dual intracellular recordings of MDHns and extracellular suction electrode recordings of the IIN1B nerve which provides motor output to the forewing. First, the wings/legs of animals were removed, and animals were placed at $4^{\circ} \mathrm{C}$ to slightly anesthetize them for mounting and surgery. A dorsal longitudinal cut was made along the thorax, and a cross sectional cut was made along the thoracic/abdominal line. The thorax was opened by placing two insect pins in the thoracic cavity, and out underneath the wing. Internal musculature and digestive organs blocking the ventral nerve cord were removed. A small piece of cuticle that rests in-between the prothoracic and pterothoracic ganglion was cut, and the musculature attached to the cuticle was also removed. The dorsal portion of the pterothoracic ganglionic sheath was carefully removed, and all peripheral nerves were cut to ensure recording stability. To further immobilize the pterothoracic ganglion during recording, the tip of a laboratory scoopula was cut so it was roughly the width of the ganglion and was placed underneath the ganglion with a very light upward pressure applied to the nerve cord. A custom-built borosilicate glass suction electrode was placed in the saline bath and a light back pressure was used to obtain a tight seal on the IIN1B nerve. This effectively allowed recording of gross wing motor

output. It is possible that the axotomized sensory afferents were still active and recorded by the suction electrode, however, it seems highly unlikely that sensory axons could generate a backpropagating spike particularly after the $\sim 45$ min delay between the initial cut and the subsequent recording. Finally, an intracellular electrode was carefully lowered to the ganglion and the primary neurites of the MDHns were targeted. If a penetrated neuron displayed the physiological characteristics consistent with the MDHn (i.e. 15 Hz tonic spike rate with a 0.1 ms spike halfwidth), a separate line containing $10^{-5} \mathrm{M}$ chlordimeform HCL (Sigma-Aldrich, 35914 ) in saline was switched on to activate the flight central pattern generator[37, 38], 
activation took $\sim 2 \mathrm{~min}$. After $\sim 10$ minutes of recording time, recorded cell was filled by passing continuous current $(0.2-2.0 \mathrm{M} \Omega)$ for at least 7 minutes.

Intracellular electrodes were pulled to produce resistance between 40-100 M $\Omega$ (Sutter Instruments, P-2000). The tips of the electrodes were filled with Alexa Fluor 568 hydrazide sodium salt for microinjection (Life Technologies, A10441), and the stems were filled with $2 \mathrm{M}$ potassium acetate. Intracellular electrodes were connected to the headstage of Axoclamp 2B (Molecular Devices) using silver/silver chloride wire. Another silver/silver chloride wire was placed in the thoracic cavity as a reference electrode. The suction electrode was constructed with standard methods with the exception that a glass capillary tube was hand pulled over an open flame to create the hourglass shape necessary for a tight seal around the nerve tip. The suction electrode was connected to an amplifier (Model 3000, A-M Systems) by silver/silver chloride wire, and another silver/silver chloride wire ran along the outside of the electrode as a reference. Signals were digitized (Digidata 1440A: Molecular Devices) and written to an external hard drive (16 bit at $20 \mathrm{kHz}$ Clampex, version 10.6: Molecular Devices).

To confirm the identity of MDHns, directly after recording and filling MDHn, dissected ganglia were placed in a 4\% N-3-dimethylaminopropyl-N'-ethylcarbodiimide (Sigma-Aldrich, 03449) for 3-4 hours at $4{ }^{\circ} \mathrm{C}$, and placed in $4 \%$ paraformaldehyde (Electron Microscopy Sciences, 15710) in $1 \%$ phosphate buffered saline (PBS: Sigma-Aldrich, SLBJ3744V) overnight. Ganglia were then washed four times in PBS with 0.5\% Triton TM X100 (PBST: Sigma-Aldrich, 110M0009V) for 15 minutes, and stored for up to 3 weeks at $4^{\circ} \mathrm{C}$ for batch processing. When enough ganglia were collected, brains were blocked in 5\% normal goat serum (NGS: Jackson Laboratories, CAT\#) for one hour, then placed in primary antibody solution (1:500 Rabbit-anti-HA Immunostar, 22939 in $5 \%$ NGS and mM sodium azide (PBSAT Fisher Scientific: S2271) overnight. Ganglia were washed four times in PBST, blocked for an hour using the blocking solution above, and placed in secondary antibody solution (1:1000 Alexa Flour 488) in PBSAT overnight. Ganglia were washed twice in PBST for 15 minutes, then twice in PBS for 15 minutes, then ran through an ascending ethanol series and cleared in methyl salicylate. Images were obtained using an Olympus FV1000 laser scanning confocal microscope.

Finally, data from confirmed MDHns were analyzed in MATLAB (Version R2016a) using custom-written scripts. Intracellular traces were binarized and compressed by a factor of 10 , then the instantaneous firing rate was calculated. The instantaneous firing rates of all recordings were smoothed using gaussian smoothing windows ranging from 2 to $1000 \mathrm{~ms}$. Firing rate changes in individual recordings that occurred over long time periods were z-score normalized, and MDHn and IIN1B firing rate was plotted using a scatter plot and the data was fitted using linear regression analysis. Epochs of wing motor output were identified by thresholding then determining where the first and last above threshold spikes occurred within each bout of wing motor output. Only bouts that had clear quiescent periods before and after each bout were included, rather than bouts of wing motor output that merged together, or CPG activity that was continuously active. Spike rates were calculated for the MDHn and IIN1B fibers between and 
within bouts, and significant changes in spike rate were determined using t-tests (paired, 1tailed).

\section{Multiunit $A L$ recordings}

To characterize the effect of HA release on odor-driven response properties of AL neurons, multi-unit recordings were made using standard methods[18, 25, 41, 49, 52]. Briefly, individual male moths were placed into $12.7 \mathrm{~mm}$ ID copper tubes, their heads fixed with soft molten dental wax. Scales were removed from the head capsule and a $2 \mathrm{~mm}^{2}$ window was opened by dissecting cuticle from the rostro-medial portion just in front and between the compound eyes. Next, the caudle section of the head capsule, where the cibarial pump muscle attaches, was surgically cut free and moved rostrally with muscle attached, then glued in place with Loctite ${ }^{\mathrm{TM}}$ quick drying gel. A small amount of occluding trachea and vascular tissue was removed to expose the brain. The completed preparation was positioned on the recording stage and standard Manduca saline was applied to keep the brain hydrated and remove hemolymph. Next, a 16-channel silicone microelectrode array $(2 \times 2$ TET; NeuroNexus Technologies, Ann Arbor, MI, USA) was placed into the AL along the caudal seam between the $A L$ and the rest of the deutocerebrum using a motorized micromanipulator (HS6, WPI). Typical placement drives the tips of these probes $\sim 600 \mu \mathrm{m}$ in depth though precise depth was unknown because of dimpling and displacement of the brain as it is impaled.

All recordings and stimulation protocols are mediated through Neuralynx Cheetah software driving a 32 channel Neuralynx hardware. All three experiments were carried out in separate groups of animals. We used a stimulation protocol which pulses odor at $20 \mathrm{~Hz}$ for $500 \mathrm{~ms}$ using a $50 \%$ duty cycle (i.e. $25 \mathrm{~ms}$ on, $25 \mathrm{~ms}$ off for a total of 10 pulses). This pulse train was repeated as a block of 5 repeats with $10 \mathrm{sec}$ between each repeat. The block of 5 pulse trains were then repeated with 2 min interstitials. Thus, every two min we collected a block of 5 pulse trains. The first block was presented followed by initiation of an experimental treatment. For the pharmacological treatments, either cimetidine $(500 \mu \mathrm{M})$ or $\mathrm{HA}(50 \mu \mathrm{M})$ was bath applied starting immediately following the first pulse train block. In both cases drug was introduced into the saline drip line and the premixed dilution replaced the clean saline supply. Yoked control groups were treated in the same manner, but the introduced saline contained no pharmacological agent. For experiments in which the neck connective was severed to axotomize the MDHns, an initial block of pulse trains was delivered to the antennae and then iridectomy scissors were placed from underneath the preparation and used to sever the neck connective. This procedure was $\sim 50 \%$ successful and non-successful cuts served as the sham control.

Unitary spike trains were sorted in a semi-automated manner using MClust[53]. Clusters of spikes were initially identified using the KlustaKwik (K. Harris) algorithm and manually inspected and cleaned. Final retention of clusters as single unit spike trains for further analysis was based on standard descriptive statistics including ISI histograms, Peak by channel by time plots, and which are automatically calculated in MClust.

Analysis: In order to determine the ability of the recorded AL unitary population to entrain to individual pulses in each pulse train, we used a previously described method whereby the integrated power around the pulse frequency $(20+/-2 \mathrm{~Hz})$ was calculated, then averaged across units, animals and pulse trains for each 2 min step in time[18, 25]. Welch's method of power spectral density was used on binarized spike train data. For parametric analysis and display, 
mean and standard error at each 2 min step were calculated in MS Excel for each experimental and corresponding control group and normalized to their respective initial integrated power by dividing power at time $=x$ by power at time $=0$, where ' $x$ ' is the specific 2 min time interval. As the control data for the two pharmacological treatments were identical, we pooled these results for statistical analysis. 1-tailed t-test comparison of experimental and control group means at each sequential step in time was used to identify onset of experimentally induced increase or decrease in tracking ability of the recorded population $(p<0.01)$.

\section{Behavior-pharmacology}

Subject Preparation: Three studies were performed to determine whether HA-r function contributes to behavioral olfactory acuity. We first identified an optimal pharmacological antagonist and dosage, we then characterized both detection and discrimination thresholds to pulsatile odor stimulation while disrupting AL HA-r function. In all cases, approximately equal numbers males and females were assigned to experimental and control conditions and standard injection and testing protocols were used[48, 54]. Moths were inserted head first into $4 \mathrm{~cm}$ long aluminum tubes (1.2 cm ID) with the head protruding out and above a $\sim 1 \mathrm{~cm}^{2}$ tab at the top of the tube. The head was immobilized to the tab with tape to stabilize it for surgery and injection. Scales on the head capsule were removed with forceps and compressed air. Small openings were made in the cuticle of the head capsule to the right side of the sagittal midline and on the anterior edge of the contralateral eye using an insect mounting pin. Teflon coated silver wire electromyographic (EMG) electrodes were then inserted through the openings contacting the cibarial pump muscle (a feeding muscle) and the contralateral eye (as a reference). Electrodes were connected to an amplifier (DAM 50, WPI Inc.). Amplified EMG signal was output to a loudspeaker and oscilloscope to monitor expression of conditioned feeding responses. A small amount of adhesive (Loctite ${ }^{\mathrm{TM}}$ ) was applied to each electrode to keep the electrodes in place during the subsequent surgery and injection process. Electrode circuit impedance was tested using a low voltage impedance meter $(\mathrm{FHC})$ to confirm adequate electrode placement; only preparations with a circuit impedance in the range of 0.1-0.9 $\mathrm{M} \Omega$ were used. Finally, the proboscis was extended and threaded through a $4 \mathrm{~cm}$ length of tubing (Tygon; $1.27 \mathrm{~mm}$ ID) leaving the distal tip of the proboscis exposed to apply sucrose solution (the unconditioned stimulus). This tubing was then attached to the larger tube with soft dental wax.

Olfactory Conditioning Protocols: For all behavioral experiments, moths were conditioned to respond to test odorants using well-established Pavlovian-based olfactory conditioning protocols[44]. These protocols produce a conditioned feeding response that can be used to assess behavioral detection[47] and discrimination thresholds[45]. For the conditioning phase of all experiments, subjects were placed in the conditioning stage $\sim 10 \mathrm{~cm}$ down wind of the olfactometer nozzle and the olfactometer output came only from the odor line. For each conditioning trial, the conditioning stimulus (a cartridge with $3 \mu$ of undiluted odor) was presented continuously for $4 \mathrm{sec}$. Three seconds into the odor stimulation, a $0.75 \mathrm{M}$ sucrose solution was applied to the partially extended proboscis, also for $4 \mathrm{sec}$. For detection threshold experiments, each animal received 6 odor-food conditioning trials, each separated by 6 min. On each trial, animals were scored for the presence or absence of feeding activity in response to CS presentation based on either movement of the proboscis or an increase in EMG activity of the feeding muscle observed on the oscilloscope and loudspeaker. During conditioning trials, conditioned responses were recorded if feeding activity occurred during the first $3 \mathrm{~s}$ of odor 
presentation, prior to application of sucrose solution. For discrimination threshold assays, a twoodor differential conditioning protocol was used and consisted of 12 trials total; 6 CS+ (odor paired with $0.75 \mathrm{M}$ sucrose solution) and $6 \mathrm{CS}$ - (odor only) trials. Two pseudorandom sequences of the CS+ and CS- trials were used to avoid stimulus sequence effects: 1$)+--+-+$ +-+--+ and 2) - + + - + - + - + + -. Furthermore, both odorants (2-hexanone and 2-octanone) were used as the CS+ and CS- odors in separate but equally sized subgroups to methodologically control for odor-dependent effects. Counterbalanced data was pooled by CS+ and CS- for analysis and display. Again, a six min inter-trial interval was maintained between trials as above and the presence of conditioned feeding responses were recorded by trial for both odors. Post conditioning, all animals were placed back into the incubator at $25^{\circ} \mathrm{C}$, and $75 \%$ $\mathrm{RH}$, then tested $24 \mathrm{hrs}$ later.

Surgery and Injection Protocols: Prior to testing, both ALs were surgically exposed for injection using standard protocols[48]. To expose the ALs a $\sim 2 \mathrm{~mm}^{2}$ piece of cuticle was removed from the anterior portion of the head capsule. Just posterior to this opening, a patch of cuticle with the cibarial pump muscle attached was then sectioned, leaving the muscles attached. This section was moved forward into the previously removed cuticle exposing the antennal lobes. A small drop of Loctite ${ }^{\mathrm{TM}}$ adhesive was used to hold the repositioned muscle and cuticle in place, allowing the muscle to function approximately normally. Some minor trachea obstructing direct access to the ALs was also removed. During surgery and post injection, the exposed brain was periodically given 3-4 droplets from a syringe of $\mathrm{pH}$ buffered Manduca physiological saline to clear any hemolymph accumulation and keep the brain hydrated. Next, a sharp quartz intracellular electrode (P2000 Sutter Instruments) was sheared at the tip to produce an $\sim 10 \mu \mathrm{m}$ ID injection needle, filled with the desired solution, then fitted to a General Valve Pico Spritzer II pressure injector. Each injection needle was calibrated to produce $\sim 1 \mathrm{nl}$ droplets. Recalibration was performed between each injection to ensure proper dose delivery. If the injection needle clogged or broke, such that actual dose was potentially incorrect, the animal was excluded, and the needle replaced.

\section{Testing Protocols}

To determine the most effective minimum dose and pharmacological agent, a dose response study was performed for two HA antagonists. Testing was conducted 15 minutes post injection based on multi-unit results (above) which indicated that this was sufficient time for the drugs produce changes in network function. Moths were conditioned, then assigned to one of $8 \mathrm{drug}$ treatment groups ( $n=60 /$ group or 360 total moths), or a matched saline control group for each drug treatment type that was randomly collected in tandem ( $n=60 /$ group or 120 total moths). Moths from control groups were injected with $\mathrm{PH}$ buffered Manduca physiological saline. Moths in drug treatment groups were injected with a dilution of one of two HA h-2 receptor antagonists: cimetidine (CIM; $50 \mu \mathrm{M}, 5 \mu \mathrm{M}, 0.5 \mu \mathrm{M}$; Sigma-Aldrich) or ranitidine hydrochloride (RAN; $50 \mu \mathrm{M}$, $5 \mu \mathrm{M}, 0.5 \mu \mathrm{M}$; Sigma-Aldrich), dissolved in the physiological saline. The highest antagonist dilution was well below previously documented concentrations associated with the emergence of secondary effects[55]. In all cases, two individuals carried out the experimental protocols; one person injected and a second person, blind to the injection contents, tested moths for conditioned responses to the test panel. All moths were tested first with an odor blank then across a log-step dilution series of the CS (2-hexanone) from 0.001 - $10 \mu \mathrm{g}$ odor diluted in $2 \mu \mathrm{l}$ mineral oil, dilutions were calculated based on density. For these first dose response studies, 
each test stimulus was presented a $4 \mathrm{~s}$ continuous stream of odor presented $\sim 10 \mathrm{~cm}$ from the antennae and at a measured flow of $275 \mathrm{ml} / \mathrm{min}$. After testing, each test subject received a small drop of $0.75 \mathrm{M}$ sucrose solution to confirm that the animal could elicit a feeding response and establishes that a lack of feeding response was not due to an inability to behave. Based on these dose-response results, we selected a single h-2 antagonist and dose for subsequent experiments (cimetidine, $50 \mu \mathrm{M}$ ).

To determine the role of AL HA-r function on olfactory sensitivity, we used a detection threshold assay[43, 47] where odor was pulsed to simulate wing beating induced flows. All animals were conditioned, injected then tested with the same dilution series as described above but for the following exceptions. First, odor was interleaved into clean air and delivered as a $20 \mathrm{~Hz}$ pulse train for a total of $4 \mathrm{~s}$ using a 10:40 ms odor:air duty cycle. Second, the odor nozzle was positioned $\sim 2-3 \mathrm{~mm}$ from a single antenna, which was restrained with small spring coil. Third, the out flow of the odor delivery nozzle was lowered to $30 \mathrm{~cm} / \mathrm{s}$ to replicate previously measured wing beat induced axial inflows past the antenna caused by wing beating in tethered moths[23]. The olfactometer produces approximately the same amplitude of flux in flow as wing beating as well as oscillating odor concentrations[43]. Moths were assigned to either a $50 \mu \mathrm{M}$ cimetidine, or a saline control group ( $n=60 /$ group); we used a double-blind injection and testing protocol.

Finally, to determine the effect of HA-r function on olfactory acuity, we performed a discrimination threshold assay, which has been detailed previously[47]. Here two odors 2 hexanone (Sigma 98\% pure) and 2-octanone (Sigma 98\% pure) were used. Again, animals were injected and tested $24 \mathrm{~h}$ post conditioning as described for the detection threshold assay, though in this case testing was over a dilution series of two odors and ranged from 10-100ug in $2 \mathrm{uL}$ of mineral odor. All moths were tested first with a pulsed odor blank, then with pulsed odor across the log-step dilution series of both CS+ and CS-.

\section{Statistical Analysis}

Behavioral data were analyzed in SAS using the general linear modeling (GLM) procedure[56]. GLM has the advantage of hierarchically partitioning variance components, thus providing a more stringent statistical test. Each drug treatment group was compared individually against a methodologically matched control group. Here, we hierarchically partitioned variance associated with the main effect of odor concentration, drug treatment, age, and sex as well as their 2 and 3way interactions with a significance value of $p<.05$. Note that the only variable of interest was the treatment by concentration interaction for the detection threshold study and the reinforcement by treatment by concentration interaction for the discrimination threshold studies. These interaction terms explicitly evaluate the effect of the drug treatment on the odor concentration-response function. Post-hoc analyses were then performed using one-tailed paired t-tests to statistically identify detection and discrimination thresholds by specific statistical comparison as previously described[43, 45, 47]. Here detection threshold was defined as the lowest concentration of odor that produced a significant increase in conditioned response probability over the response to blanks $(p<.01)$. Discrimination threshold was defined as the lowest concentration for which there was a significant differential response between the CS+ and CS- $(p<0.01)$. All inset regressions for conditioning data are $2^{\text {nd }}$ order polynomial functions to highlight acquisition of the CR. All test results are displayed with inset $3^{\text {rd }}$ order polynomial regressions to highlight the odor concentration-response functions. 
A one-tailed paired t-test was used to statistically identify detection and discrimination thresholds by specific statistical comparison as previously described[43, 45, 47]. Here detection threshold was defined as the lowest concentration of odor that produced a significant increase in conditioned response probability over the response to blank stimuli $(p<.01)$. Discrimination threshold was defined as the lowest concentration at which there was a significant differential response between the CS+ and CS- $(p<0.01)$. All inset regressions for conditioning data are $2^{\text {nd }}$ order polynomial functions to highlight acquisition of the CR. All test results are displayed with inset $3^{\text {rd }}$ order polynomial regressions to highlight the odor concentration-response functions.

\section{Supplemental Behavioral Results}

The goal of the behavior pharmacological studies was to determine the role of HA on olfactory function. The MDHns are the exclusive source of HA to the ALs[30], thus our approach was to train moths to elicit a conditioned feeding response to target odors, pharmacologically block HA$r$ activation using focal injections, then compare detection thresholds as a function of pharmacological treatment. In our first series of experiments we conditioned four groups of moths to respond to 2 -hexanone then tested using standard $4 \mathrm{sec}$ continuous stimuli. Figure S1A displays acquisition curves for these groups, which include three concentrations of cimetidine groups and a saline-vehicle injected control group. Mean response values for the acquisition phase were normalized according to the initial responsiveness to the blank stimulus for each group. Note that inset regression lines indicate that acquisition of a conditioned feeding response to odor occurred at approximately the same rate and to the same degree across groups.

Cimetidine: First, to evaluate the effect of increasing cimetidine dose we statistically compared the saline control group to each drug dose group. Results established that the higher two doses of cimetidine significantly reduced responses to odor overall (SI Fig.1B; $n=60 /$ group; $p<0.01$ ). Next, to evaluate differences in concentration response as a function of drug treatment we used general linear modeling. This model was significant (GLM; $n=60 / g r o u p ; p<.001$ ) and explained approximately $35 \%$ of the variance in conditioned feeding response. Importantly we observed a drug treatment by odor concentration interaction $(p=0.014)$ indicating different concentration response functions and possibly different detection thresholds. Post hoc comparison of CR probability for each odor concentration to the blank for both the drug treated and matched saline-vehicle control group indicated a significantly higher detection threshold for the drug treated group for the highest drug dose ( $50 \mu \mathrm{M}$ cimetidine; Fig. 1C). However, no differences detection threshold was found at the lower two dosages (Fig. 1D and 1E). Across all experimental and control groups, the detection threshold was determined to be $1 \mu \mathrm{g}$, except for the $50 \mu \mathrm{M}$ cimetidine treatment which was one log step higher.

Ranitidine: By comparison SI Figure 2 displays acquisition and dose response functions for 3 concentrations of ranitidine-injected groups along with their matched control groups. Responses during acquisition were normalized to the initial responsiveness for each group (Fig 2A). Inset regression lines indicate that all groups acquired the conditioned responses at the approximately same rate and to the same degree. To evaluate the effect of dose, we again statistically compared the saline control group with each drug dose. Results established that only the highest dose of ranitidine significantly reduced responses to odor overall (SI Fig. 2B; $n=60 ; p<0.05)$. The general linear model explaining variation in conditioned response 
probability between groups during detection threshold testing was significant $(p<.0001)$ and explained approximately $37 \%$ of the variance. We again found a significant interaction of drug treatment by odor concentration $(p<0.05)$. We then compared detection thresholds for each treatment experimental and control group. SI Figure $2 \mathrm{C}$ displays an increased detection threshold for the $50 \mu \mathrm{M}$ treatment group (paired t-test; $n=60 ; p<.01$ ). This analysis also indicated an increase in detection threshold for the $5 \mu \mathrm{M}$ injected moths (Fig. 2D; paired t-test; $n=60$; $\mathrm{p}<.01$ ). By comparison, the $0.5 \mu \mathrm{M}$ treatment group, produced the same detection threshold of 1 $\mu \mathrm{g} / 2 \mu \mathrm{L}$ as the control groups. Taken together with the cimetidine experiment these results indicate a generalized disruption of olfactory sensitivity when injecting competitive HA h-2 receptor antagonists into the $\mathrm{AL}$. 
Figure legends

Figure 1: MDH activity is correlated with flight motor patterns. $(A)$ Schematic of key components of the moth CNS including the antennal lobes (AL), subesophageal zone (SEZ) and antennal mechanosensory and motor center (AMMC) and the pterothoracic ganglion (PTG) which includes the fused mesothoracic and metathoracic neuromeres. Also highlighted is our experimental approach, which included the simultaneous intracellular recording of an MDHn (blue) and suction electrode recording of the IIN1b nerve fiber (red) while wing motor output is driven from the flight central pattern generator via bath applied chlordimeform $(50 \mu \mathrm{M})$. (B) HA immunolabeling (green) of the MDHns with the intracellularly recorded $\mathrm{MDHn}$ filled with Alexa 568 (magenta). Inset below zoom of two distinct cell bodies labeled. Left: both laser channels. Center: Alexa 568 channel showing a single filled cell body and primary neurite. Right: HA channel showing the two cell bodies and primary neurites of the MDHn pair. Complete spatial overlap confirms the recording was of an MDHn. $\left(C_{i}\right)$ Superimposition of the smoothed instantaneous spike rate of the recorded $\mathrm{MDHn}$ (blue) and the raw extracellular recording of the IIN1b fiber (red). Inset dashed rectangle highlights the time sample shown in $\left(C_{i i}\right)$ which shows the raw spike trains for both traces. Note that the $\mathrm{MDH}$ spike rate always increases just prior to and during bouts of wing motor output. $(D)$ Plot of z-score normalized spike rate for MDH (blue) and IIN1b (red) across 10 min of continuous recording demonstrating that as IIN1b activity increases over time, so too does MDH spike rate $(r=0.71)$. ( $E$ ) Scatterplot of $z$-score normalized spike rate of MDH and IIN1b. Inset linear regression $\left(n=4\right.$ recordings/738 points; $R^{2}$ $=0.09, r=0.30) .(F)$ Mean spike rate from epochs where the IIN1b was quiet versus producing wing motor output from the recording highlighted in $\mathrm{C}$. Inset error bars represent the standard error. Inset statistical comparisons between states indicates corresponding significant increase in both IIN1b and MDH (Welch's t-test; $n=8$ recording segments; $p<0.05$ ). ( $G$ ) Crosscorrelation between MDH and IIN1b firing rates using Gaussian smoothing windows ranging in width from $2 \mathrm{~ms}$ to $1000 \mathrm{~ms}$. Note that for smoothing widows within typical spike integration times $(2-5 \mathrm{~ms})$ there is no correlation between measures.

Figure 2: HA enhances entrainment of $A L P N s$ to rapidly pulsed odor. To evaluate the effect of MDHn HA release on the ability of AL PNs to entrain to pulsed stimuli, we performed three experiments, each in separate groups of animals. For all experiments, multi-channel electrodes were placed into the $A L$ and multi-unit recordings were made while the ipsilateral antenna was stimulated with a block of five $500 \mathrm{~ms}$ long stimulation at $20 \mathrm{~Hz}$ pulse trains every two minutes for a total of 15 presentations. After the first block of pulse trains animals were challenged with an experimental treatment. $(A)$ In the first group, to disrupt HA-r function we bath applied $50 \mu \mathrm{M}$ cimetidine $(\mathrm{CIM})$ in saline vehicle continuously over the course of the experiment. $(B)$ In the second group of animals, to remove intrinsic HA input from the MDHn's the neck connective was cut thereby axotomizing the MDHns. $(C)$ in the last group of animals, direct bath application of $\mathrm{HA}(50 \mu \mathrm{M})$ in saline vehicle was used to simulate increased MDHn output during flight. Columns $(A-C)$ : (i) Schematic of each experiment. (ii) Exemplar peristimulus rasters (top) and histograms (bottom) for the baseline responses (before) and during/after treatments. (iii) Mean integrated power from $18-22 \mathrm{~Hz}$ by time across all recorded neurons that entrained to the pulsed odor at some point during each experiment. Error bars represent the standard error. Results plotted as a function of time since treatment. Power was normalized by dividing mean power from each block by the mean baseline (pre-treatment block) power. Inset arrows indicated the first block where there was a significant difference in power between experimental and control treatments (Welch's t-test for two samples with unequal variance; $p<0.05$ ). Inset 
regressions are second order polynomials. Inset red rectangle $\left(A_{i i}\right)$ highlights the loss of responses to the first two pulses as a consequence of cimetidine relative to pre-treatment.

Figure 3: HA-r blockade disrupts behavioral measures of olfactory acuity. ( $A$ ) Acquisition of the conditioned feeding response to a single odor (2-hexanone) as a function of conditioning trial for groups of moths in the detection threshold assay. Twenty-four hours later, one group of moths was bilaterally injected with either $50 \mu \mathrm{M}$ cimetidine (CIM) in saline vehicle or the saline vehicle without drug (Saline) in a blind manner then tested. $(B)$ Conditioned feeding response as a function of odor concentration for the CIM and Saline groups. Inset open and filled arrowheads indicate detection threshold concentrations, for the Saline and CIM groups respectively, as defined by the lowest concentration odor yielding a significant increase in response relative to the blank (1-tailed paired t-test; $n=60 ; p<0.001$. (C) Acquisition of the differential conditioned feeding response to the CS+ and CS- stimuli for CIM and saline injected groups. Moths were first differentially conditioned to one of the two odorants (2-hexanone or 2-octanone). Both odors were used as the CS+ and CS- in separate but equally sized groups to counterbalance odordependent effects; for display, data was pooled by CS+ and CS-. (D) Discrimination index ((CS)-(CS+)) displayed by concentration for the CIM and Saline injected groups. Inset open and filled arrowheads indicate discrimination threshold, the concentration at which there was a significant differential response to the CS+ and CS- odors using 1-tailed paired t-tests (saline controls: $p=0.03 ; n=46$; CIM injected: $p=0.05 ; n=43$ ). All inset regression lines are $3^{\text {rd }}$ order polynomials and all error bars represent the standard error.

\section{Supplemental Figure Legends}

SF1: HA increases PN entrainment and overall spiking response magnitude. Results based on 22 units recorded in 3 moths from the multiunit study. $(A)$ Mean raw power spectral densities from pulses responses recorded over thirty minutes. For display purposes, we collapsed the 15 timesteps of the $30 \mathrm{~min}$ experiment into five time periods (i.e. three steps/period). Shown are frequencies from $5-50 \mathrm{~Hz}$. $(B)$ The same mean power spectra displayed in A but normalized by the mean power within each period to correct for broad global changes in power. Note that in either case, power at the pulsing frequency consistently goes up primarily at the pulsing frequency and this power systematically increases over the 30 min course of the experiment. (C) Mean spike rate during the 600 ms duration of the pulse driven response. Inset asterisks indicate a significant increase in spike rate during the response as compared to the initial response (1-tailed matched sample t-test; $p<0.05)$. (D) Mean spontaneous spike rate of the same population of units during the $600 \mathrm{~ms}$ preceding each pulse train. Note that initially, odor responses drove fewer spikes than were spontaneously evoked.

SF2: Bilateral cimetidine injection increases detection thresholds. $(A)$ Acquisition of a conditioned feeding response to an odor (2-hexanone) as a function of six consecutive forward paired odor-food conditioning trials. Inset are $2^{\text {nd }}$ order polynomials for each treatment, error bars are standard errors and for clarity shown only for the control group. $(B)$ Mean conditioned feeding response to odor during the test phase of the experiment as a function of drug dose (control data excluded) and collapsed across odor concentration. Inset letters indicate significant changes in conditioned feeding response to odor (one tailed paried t-test; $p<0.05$ ). $(C-E)$ Conditioned feeding response as a function of odor concentration for saline vehicle control (saline) vs saline vehicle with cimetidine (CIM). Inset regressions are $3^{\text {rd }}$ order 
polynomial functions. Open and closed arrows indicate detection threshold for saline control and CIM injected groups respectively. (C) $50 \mu \mathrm{M}$ CIM; (D) $5 \mu \mathrm{M} \mathrm{CIM;}(E) 0.5 \mu \mathrm{M} \mathrm{CIM}$.

SF3: Bilateral ranitidine injection increases detection thresholds. $(A)$ Acquisition of a conditioned feeding response to an odor (2-hexanone) as a function of six consecutive forward paired odorfood conditioning trials. Inset are $2^{\text {nd }}$ order polynomials for each treatment, error bars are standard errors and for clarity shown only for the control group. (B) Mean conditioned feeding response to odor during the test phase of the experiment as a function of drug dose (control data excluded) and collapsed across odor concentration. Inset letters indicate significant changes in conditioned feeding response to odor (one tailed paried t-test; $p<0.05)$. $(C-E)$ Conditioned feeding response as a function of odor concentration for saline vehicle control (saline) vs cimetidine (CIM) in saline. Open and closed arrows indicate detection threshold for saline and CIM injected groups respectively. Inset regressions are $3^{\text {rd }}$ order polynomial functions. (C) $50 \mu \mathrm{M}$ CIM; (D) $5 \mu \mathrm{M}$ CIM; (E) $0.5 \mu \mathrm{M}$ CIM. 


\section{References}

1. Sommer MA, Wurtz RH (2008) Visual perception and corollary discharge. In: Perception. pp 408-418

2. Crapse TB, Sommer M a (2008) Corollary discharge across the animal kingdom. Nat Rev Neurosci 9:587-600. doi: 10.1038/nrn2457

3. Roy JE (2004) Dissociating self-generated from passively applied head motion: neural mechanisms in the vestibular nuclei. J Neurosci 24:2102-2111 . doi: 10.1523/JNEUROSCI.3988-03.2004

4. Poulet JF a, Hedwig B (2002) A corollary discharge maintains auditory sensitivity during sound production. Nature 418:872-876. doi: 10.1038/nature00919

5. Sommer MA, Wurtz RH (2006) Influence of the thalamus on spatial visual processing in frontal cortex. Nature 444:374-377. doi: 10.1038/nature05279

6. Cavanaugh J, Berman RA, Joiner WM, Wurtz RH (2016) Saccadic corollary discharge underlies stable visual perception. J Neurosci 36:31-42. doi: 10.1523/JNEUROSCI.2054-15.2016

7. Rath-Wilson K, Guitton D (2015) Oculomotor control after hemidecortication: A single hemisphere encodes corollary discharges for bilateral saccades. Cortex 63:232249 . doi: 10.1016/j.cortex.2014.08.020

8. Poulet JFA, Hedwig B (2006) The cellular basis of a corollary discharge. Science 311:518-22 . doi: 10.1126/science.1120847

9. Schneider DM, Nelson A, Mooney R (2014) A synaptic and circuit basis for corollary discharge in the auditory cortex. Nature 513:189-194 . doi:

10.1038/nature 13724

10. Montgomery JC, Bodznick D (1994) An adaptive filter that cancels self-induced noise in the electrosensory and lateral line mechanosensory systems of fish. Neurosci Lett 174:145-148 . doi: 10.1016/0304-3940(94)90007-8

11. Moore JD, Deschênes M, Furuta T, et al (2013) Hierarchy of orofacial rhythms revealed through whisking and breathing. Nature 497:205-10 . doi: 10.1038/nature12076

12. Ford JM, Mathalon DH, Roach BJ, et al (2013) Neurophysiological evidence of corollary discharge function during vocalization in psychotic patients and their nonpsychotic first-degree relatives. Schizophr Bull 39:1272-1280 . doi: $10.1093 /$ schbul/sbs129

13. Brown P (2003) Oscillatory nature of human basal ganglia activity: Relationship to the pathophysiology of parkinson's disease. Mov. Disord. 18:357-363

14. Meek PM, Schwartzstein RM, Adams L, et al (1999) Dyspnea: mechanisms, assessment, and management: a consensus statement. Am J Respir Crit Care Med 159:321-340

15. Halpern BP (1983) Tasting and smelling as active, exploratory sensory processes. Am J Otolaryngol Neck Med Surg 4:246-249 . doi: 10.1016/S01960709(83)80066-0

16. Walsh RR (1956) Single cell spike activity in the olfactory bulb. Am J Physiol 
186:255-257

17. Macrides F, Chorover SL (1972) Olfactory bulb units: activity correlated with inhalation cycles and odor quality. Science (80- ) 175:84-87 . doi:

10.1126/science.175.4017.84

18. Houot B, Burkland R, Tripathy S, Daly KC (2014) Antennal lobe representations are optimized when olfactory stimuli are periodically structured to simulate natural wing beat effects. Front Cell Neurosci 8:159 . doi: 10.3389/fncel.2014.00159

19. Connelly T, Yu Y, Grosmaitre X, et al (2014) G protein-coupled odorant receptors underlie mechanosensitivity in mammalian olfactory sensory neurons. Proc Natl Acad Sci U S A 1-6 . doi: 10.1073/pnas.1418515112

20. Obara Y (1979) Bombyx mori mationg dance: An essential in locationg the female. Appl Entomol Zool 14:130-132 . doi: 10.1303/aez.14.130

21. Loudon C, Koehl M a (2000) Sniffing by a silkworm moth: wing fanning enhances air penetration through and pheromone interception by antennae. J Exp Biol 203:29772990

22. Bau J, Justus KA, Cardé RT (2002) Antennal resolution of pulsed pheromone plumes in three moth species. J Insect Physiol 48:433-442 . doi: 10.1016/S00221910(02)00062-8

23. Sane SP, Jacobson NP (2006) Induced airflow in flying insects II. Measurement of induced flow. J Exp Biol 209:43-56 . doi: 10.1242/jeb.01958

24. Sane SP, Dieudonné A, Willis MA, Daniel TL (2007) Antennal mechanosensors mediate flight control in moths. Science 315:863-866 . doi: 10.1126/science.1133598

25. Tripathy S, Peters O, Staudacher E, et al (2010) Odors pulsed at wing beat frequencies are tracked by primary olfactory networks and enhance odor detectio. Front Cell Neurosci 4:1-14 . doi: 10.3389/neuro.03.001.2010

26. Baker TC, Willis MA, Haynes KF, Phelan PL (1985) A pulsed cloud of sex pheromone elicits upwind flight in male moths. Physiol Entomol 10:257-265. doi: 10.1111/j.1365-3032.1985.tb00045.x

27. Willis MA, Baker TC (1984) Effects of intermittent and continuous pheromone stimulation on the flight behaviour of the oriental fruit moth, Grapholita molesta. Physiol Entomol 9:341-358. doi: 10.1111/j.1365-3032.1984.tb00715.x

28. Szyszka P, Gerkin RC, Galizia CG, Smith BH (2014) High-speed odor transduction and pulse tracking by insect olfactory receptor neurons. Proc Natl Acad Sci U S A 111:16925-30. doi: 10.1073/pnas.1412051111

29. Homberg $U$ (1994) Distribution of neurotransmitters in the insect brain. Fischer, Stuttgart [etc.]

30. Bradley SP, Chapman PD, Lizbinski KM, et al (2016) A flight sensory-motor to olfactory processing circuit in the moth Manduca sexta. Front Neural Circuits 10:5. doi: 10.3389/fncir.2016.00005

31. Hardie RC (1988) Effects of antagonists on putative histamine receptors in the first visual neuropile of the housefly (i). J Exp Biol 138:221-241 
32. Hardie RC (1989) A histamine-activated chloride channel involved in neurotransmission at a photoreceptor synapse. Nature 339:704-706

33. Roeder T (2003) Metabotropic histamine receptors - Nothing for invertebrates? Eur J Pharmacol 466:85-90 . doi: 10.1016/S0014-2999(03)01553-X

34. Lofstedt C, Hansson BS, Petersson E, et al (1994) Pheromonal secretions from glands on the 5th abdominal sternite of hydropsychid and rhyacophilid caddisflies (Trichoptera). J Chem Ecol 20:153-170 . doi: 10.1007/BF02065998

35. Chapman PD, Bradley SP, Haught EJ, et al (2017) Co-option of a motor-tosensory histaminergic circuit correlates with insect flight biomechanics. Proc R Soc B Biol Sci 284:

36. Farooqui T, Robinson K, Vaessin H, Smith BH (2003) Modulation of early olfactory processing by an octopaminergic reinforcement pathway in the honeybee. $\mathrm{J}$ Neurosci 23:5370-5380

37. Kinnamon SC, Klaassen LW, Kammer AE, Claassen D (1984) Octopamine and chlordimeform enhance sensory responsiveness and production of the flight motor pattern in developing and adult moths. J Neurobiol 15:283-293

38. Vierk R, Pflueger HJ, Duch C (2009) Differential effects of octopamine and tyramine on the central pattern generator for Manduca flight. J Comp Physiol A Neuroethol Sensory, Neural, Behav Physiol 195:265-277 . doi: 10.1007/s00359-0080404-5

39. Ando N, Wang $\mathrm{H}$, Shirai $\mathrm{K}$, et al (2011) Central projections of the wing afferents in the hawkmoth, Agrius convolvuli. J Insect Physiol 57:1518-1536 . doi:

10.1016/j.jinsphys.2011.08.002

40. Eaton JL (1974) Nervous system of the head and thorax of the adult tobacco hornworm, Manduca sexta (Lepidoptera: Sphingidae). Int J Insect Morphol Embryol 3:4766 . doi: 10.1016/S0020-7322(74)81006-8

41. Daly KC (2004) Molecular features of odorants systematically influence slow temporal responses across clusters of coordinated antennal lobe units in the moth Manduca sexta. J Neurophysiol 92:236-254 . doi: 10.1152/jn.01132.2003

42. Lei $\mathrm{H}$, Reisenman $\mathrm{CE}$, Wilson $\mathrm{CH}$, et al (2011) Spiking patterns and their functional implications in the antennal lobe of the tobacco hornworm Manduca sexta. PLoS One 6: . doi: 10.1371/journal.pone.0023382

43. Daly KC, Kalwar F, Hatfield M, et al (2013) Odor detection in Manduca sexta is optimized when odor stimuli are pulsed at a frequency matching the wing beat during flight. PLoS One 8:1-18 . doi: 10.1371/journal.pone.0081863

44. Daly KC, Smith BH (2000) Associative olfactory learning in the moth Manduca sexta. J Exp Biol 203:2025-2038

45. Daly KC, Carrell LA, Mwilaria E (2008) Characterizing psychophysical measures of discrimination thresholds and the effects of concentration on discrimination learning in the moth Manduca sexta. Chem Senses 33:95-106 . doi: 10.1093/chemse/bjm068

46. Daly KC, Bradley S, Chapman PD, et al (2015) Space takes time: concentration dependent output codes from primary olfactory networks rapidly provide additional 
information at defined discrimination thresholds. Front Cell Neurosci 9:515 . doi: 10.3389/fncel.2015.00515

47. Daly KC, Carrell L a, Mwilaria E (2007) Detection versus perception: physiological and behavioral analysis of olfactory sensitivity in the moth (Manduca sexta). Behav Neurosci 121:794-807 . doi: 10.1037/0735-7044.121.4.794

48. Mwilaria EK, Ghatak C, Daly KC (2008) Disruption of GABAA in the insect antennal lobe generally increases odor detection and discrimination thresholds. Chem Senses 33:267-281 . doi: 10.1093/chemse/bjm085

49. Daly KC, Christensen $\mathrm{T}$ a, Lei $\mathrm{H}$, et al (2004) Learning modulates the ensemble representations for odors in primary olfactory networks. Proc Natl Acad Sci U S A 101:10476-10481 . doi: 10.1073/pnas.0401902101

50. Christensen T a, Waldrop BR, Hildebrand JG (1998) Multitasking in the olfactory system: context-dependent responses to odors reveal dual GABA-regulated coding mechanisms in single olfactory projection neurons. J Neurosci 18:5999-6008

51. Christensen T a, Waldrop BR, Harrow ID, Hildebrand JG (1993) Local interneurons and information-processing in the olfactory glomeruli of the moth Manduca sexta. J Comp Physiol a-Sensory Neural Behav Physiol 173:385-399 . doi: 10.1007/BF00193512

52. Daly KC, Galán RF, Peters OJ, Staudacher EM (2011) Detailed characterization of local field potential oscillations and their relationship to spike timing in the antennal lobe of the moth Manduca sexta. Front Neuroeng 4:12 . doi: 10.3389/fneng.2011.00012

53. Redish AD, Schmitzer-Torbert NC (2002) MCLUST spike sorting toolbox, version 3.0

54. Gage SL, Daly KC, Nighorn A (2013) Nitric oxide affects short-term olfactory memory in the antennal lobe of Manduca sexta. J Exp Biol 216:3294-3300 . doi: 10.1242/jeb.086694

55. Cannon KE, Fleck MW, Hough LB (2004) Effects of cimetidine-like drugs on recombinant GABAA receptors. Life Sci 75:2551-2558 . doi: https://doi.org/10.1016/j.lfs.2004.05.020

56. Cohen J, Cohen P, West SG, Aiken LS (2013) Applied multiple regression/correlation analysis for the behavioral sciences. Routledge 


\section{Figure 1:}
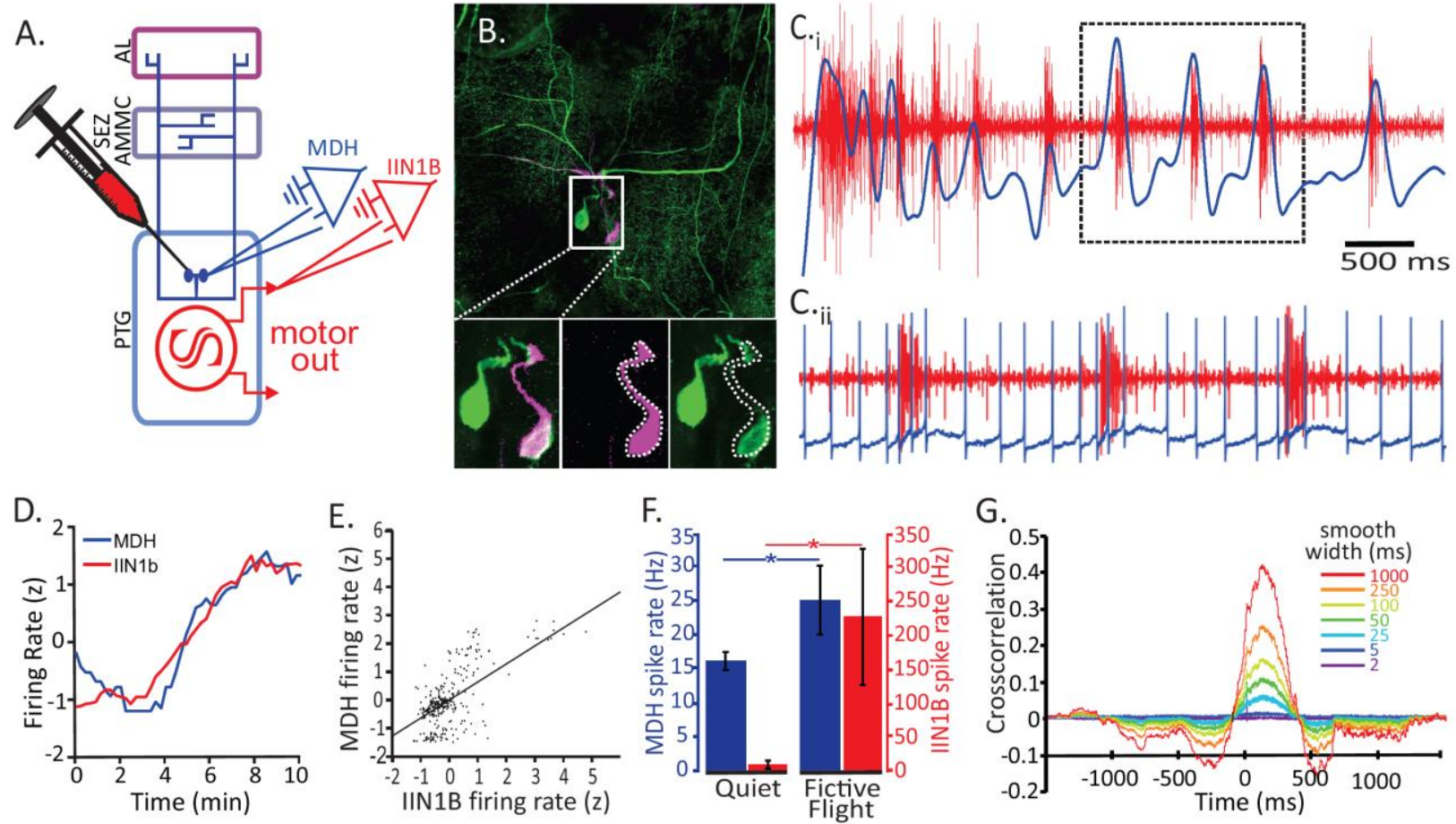

G.

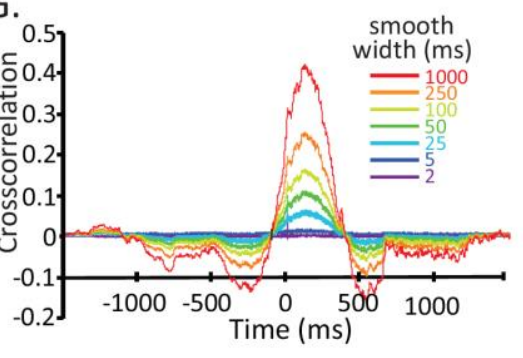


Figure 2:

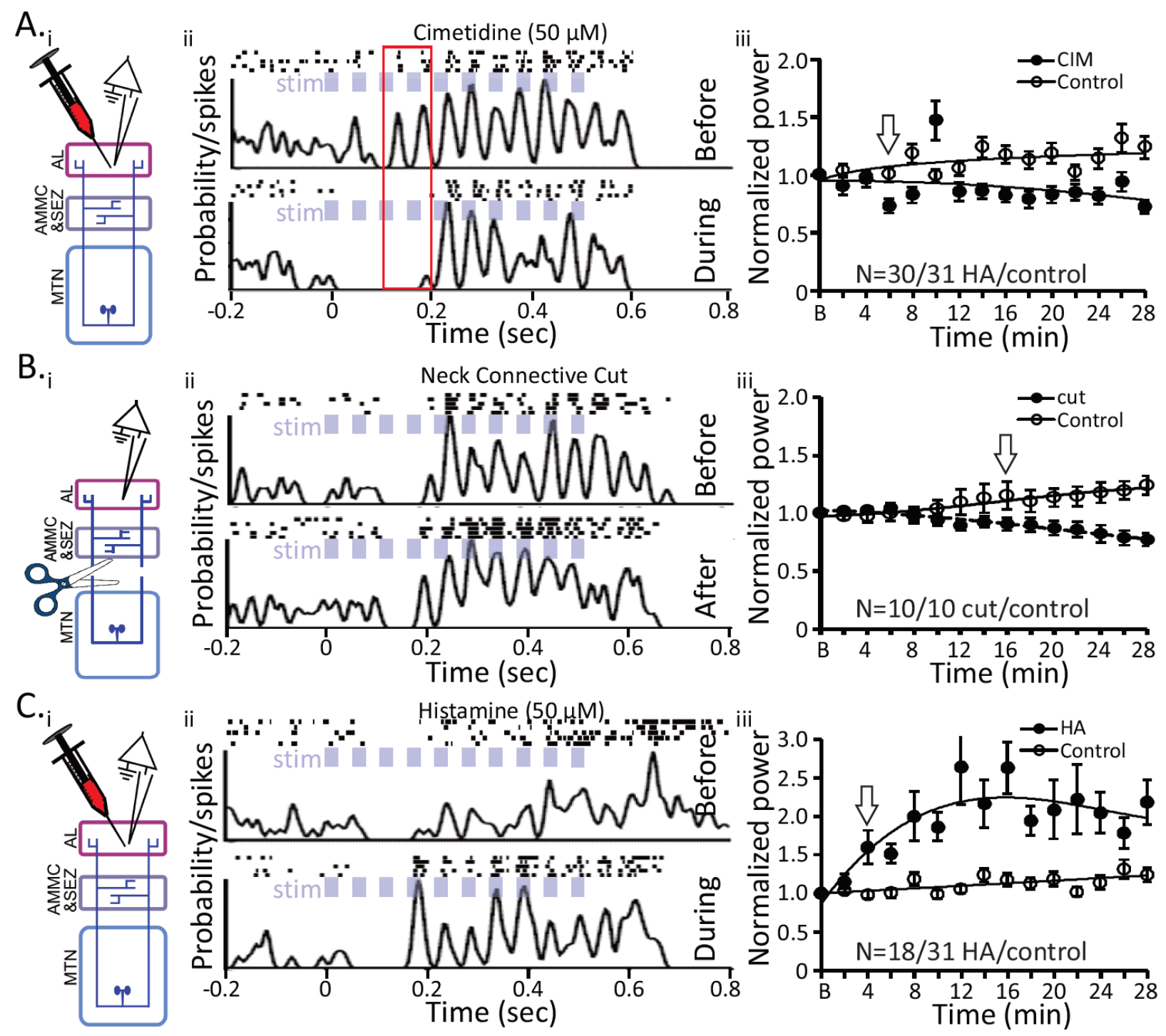


Figure 3:
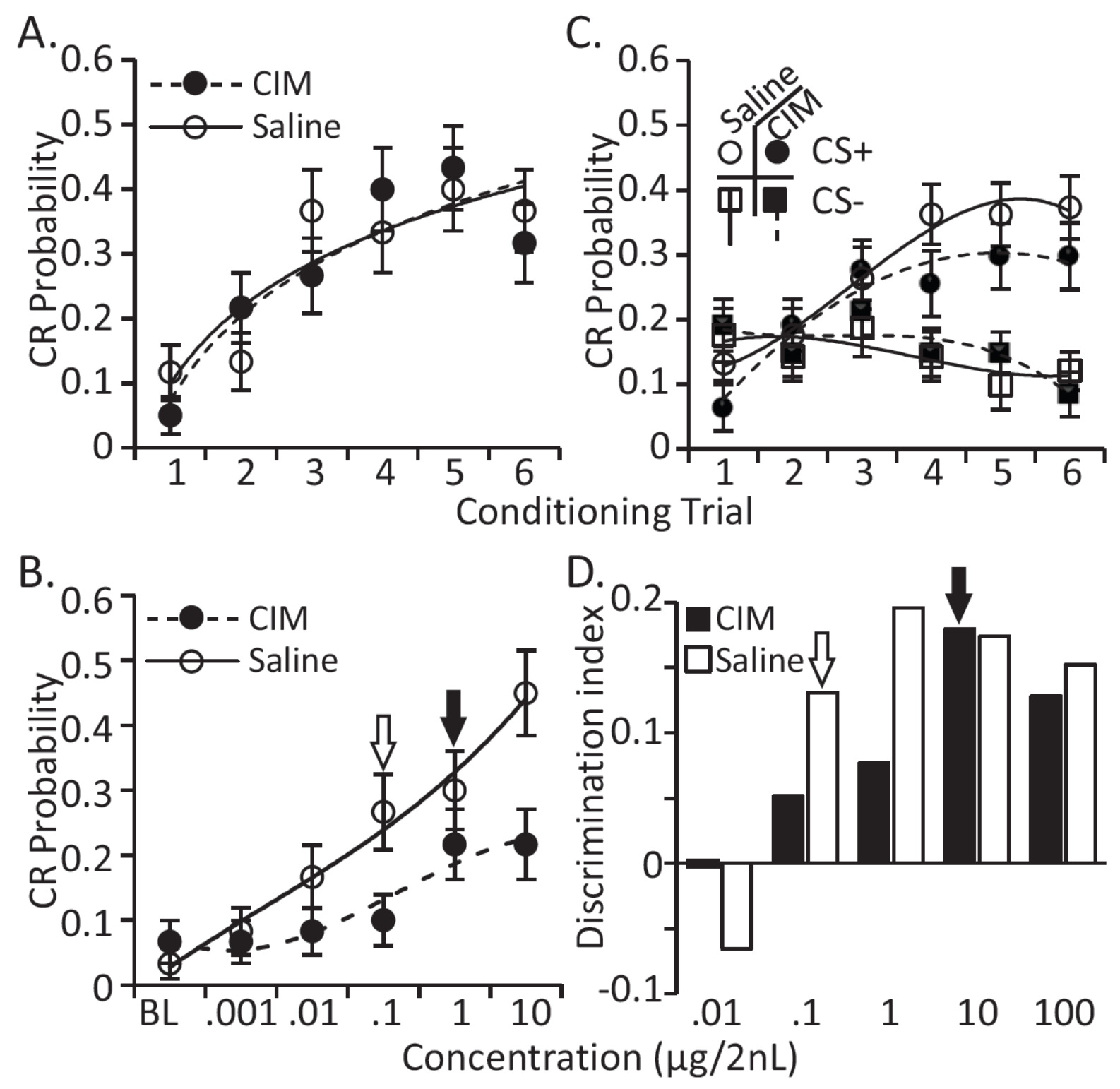
SI Figure 1:
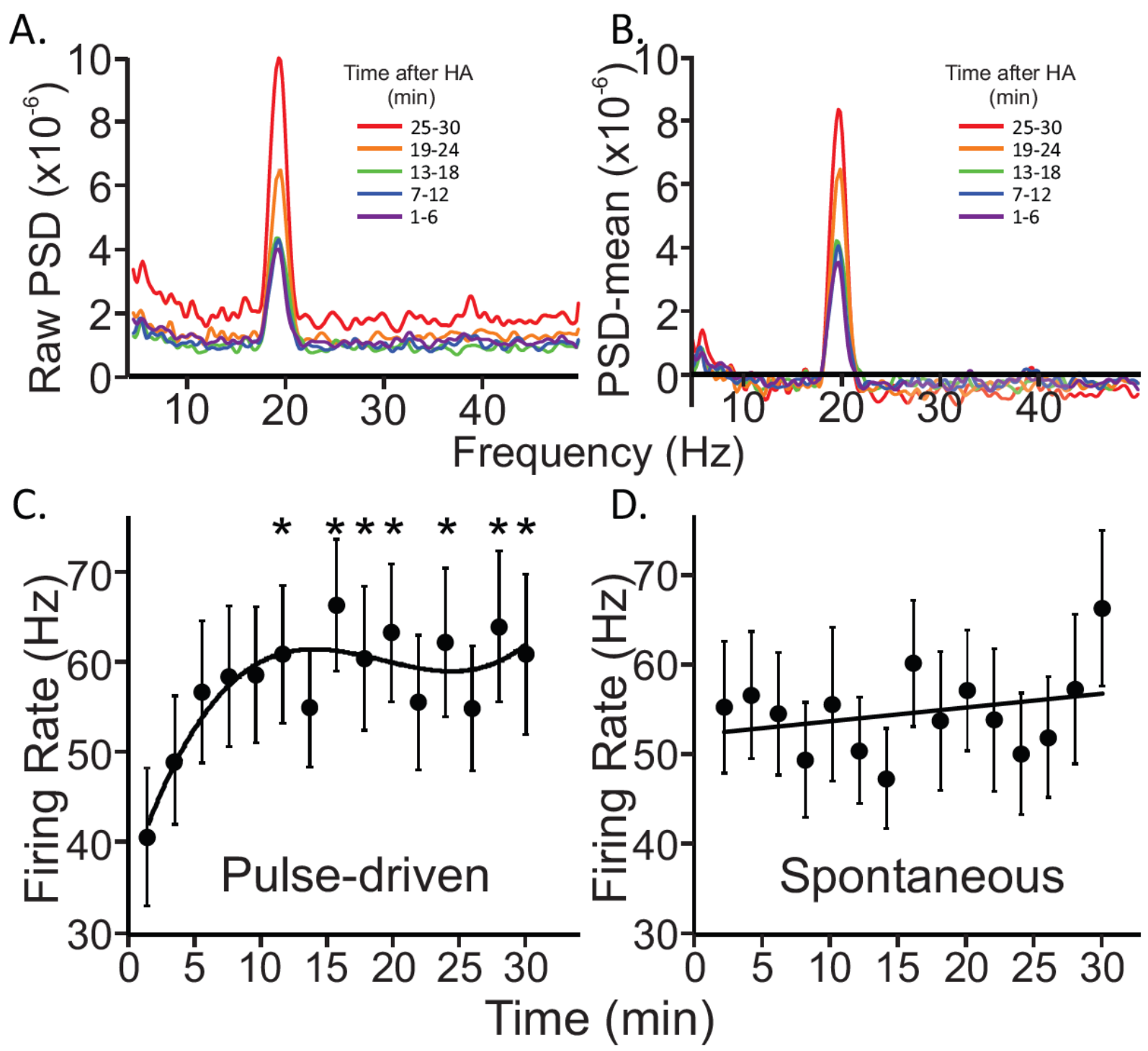


\section{SI Figure 2:}
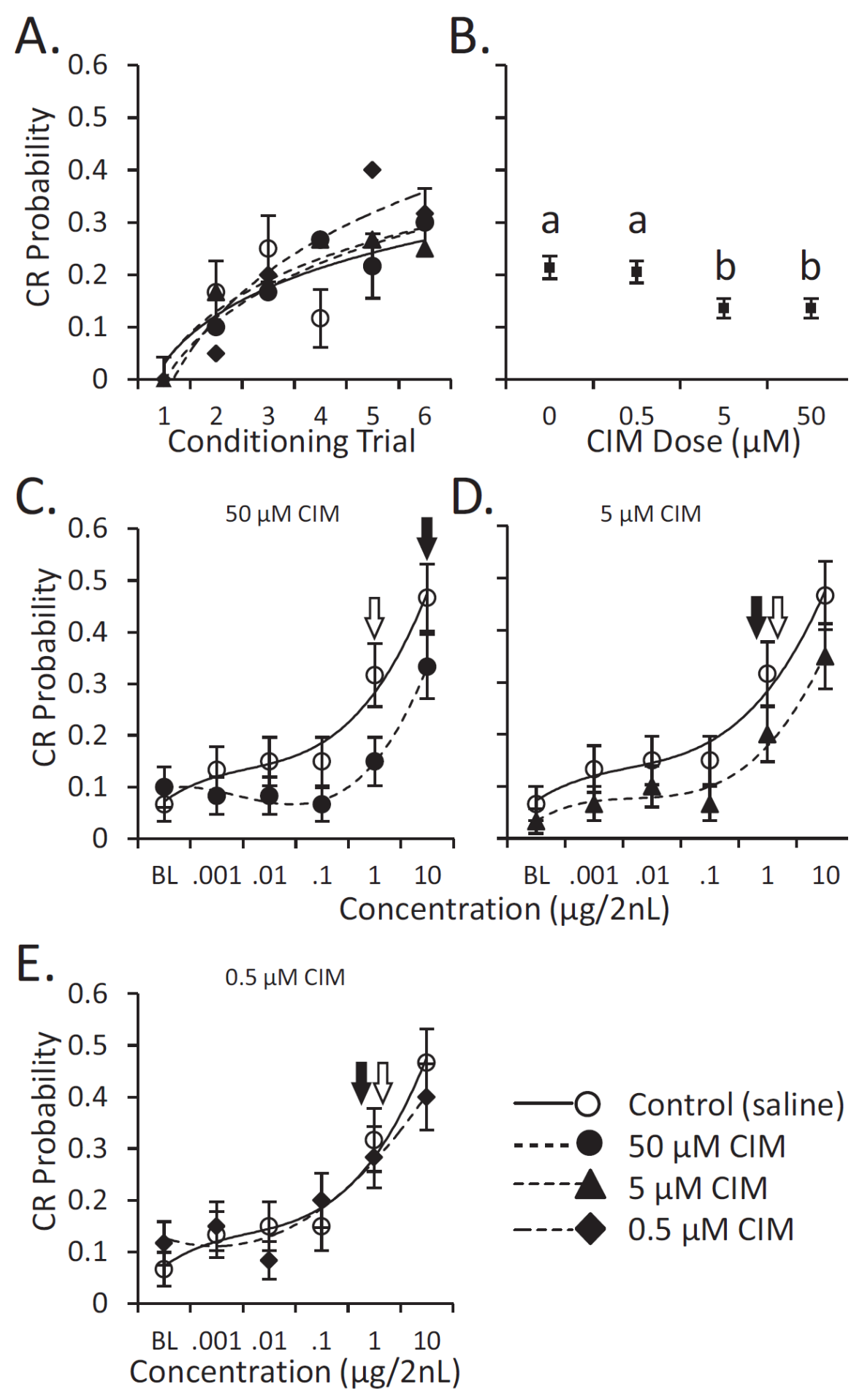


\section{SI Figure 3:}

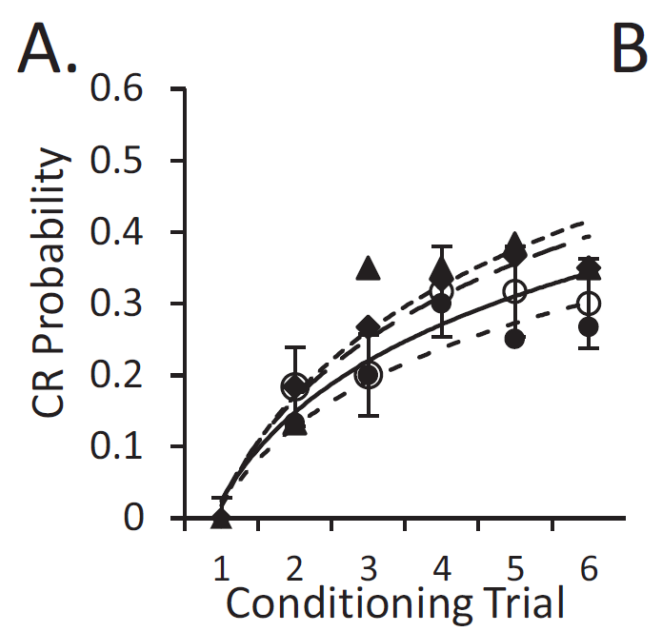

B.
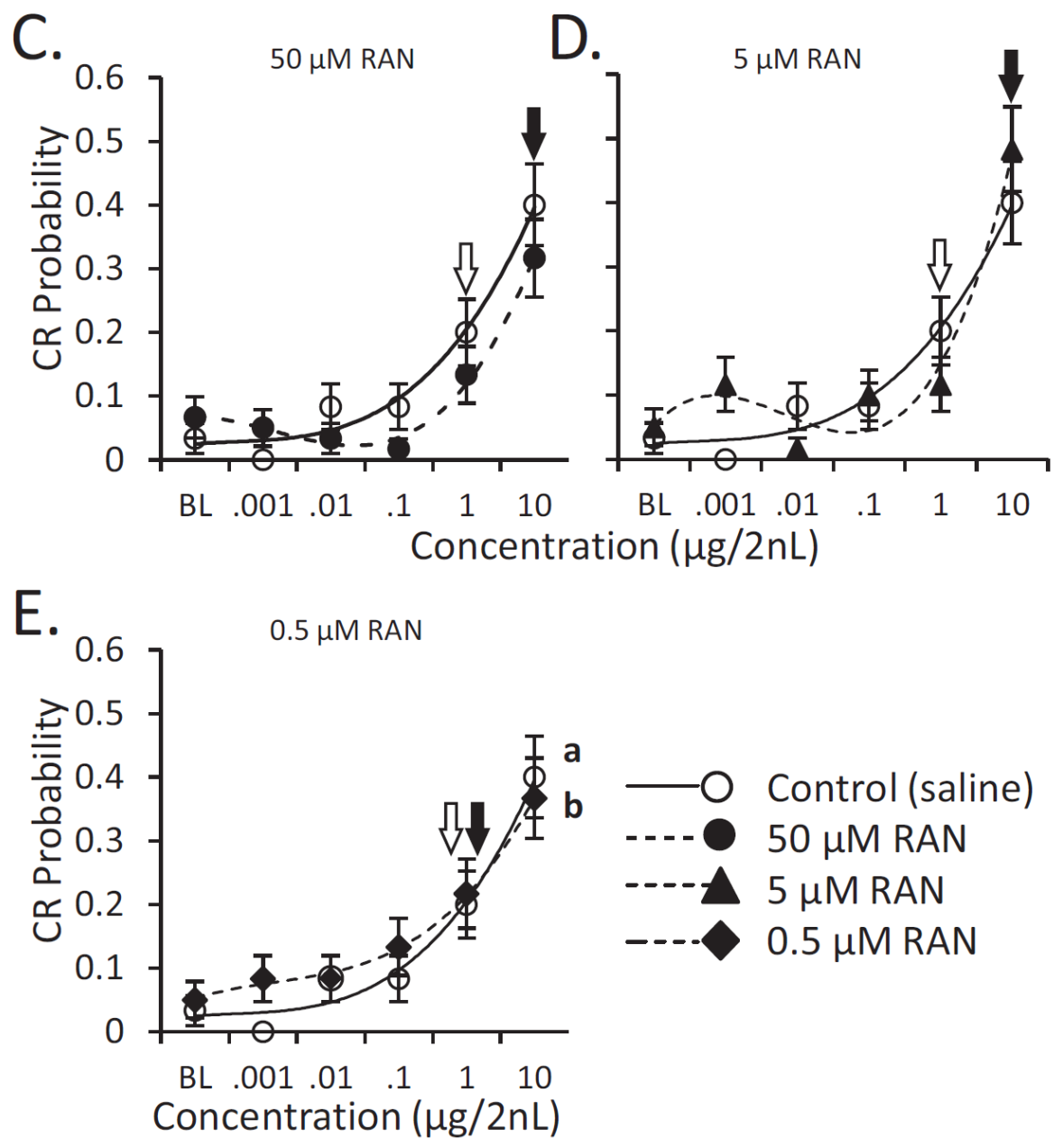


\section{Appendix C:}

Title: Evolutionary Co-option of an Ancient Motor to Sensory Histaminergic Circuit Correlates with Changes in Insect Flight Biomechanics.

Authors: Phillip D. Chapman, Samual P. Bradley, Erica J. Haught, Kassandra E. Riggs, Mouaz M. Haffar, Kevin C. Daly, Andrew M. Dacks

Author Affiliation: Department of Biology, West Virginia University, Morgantown, West Virginia, 26506, USA.

\section{Corresponding Author:}

Phillip Chapman pchapma4@mix.wvu.edu 


\section{ABSTRACT}

Nervous systems evolve in a variety of ways to adapt to shifts in behavioral ecology. One form of adaptation is neural exaptation in which neural circuits are co-opted to perform additional novel functions. In this study, we describe the co-option of a pair of motor-to-sensory histaminergic neurons into an additional sensory processing network to support odor-guided behaviors. Many moths beat their wings during the approach to an odor source, even if not flying. Wing beating can cause strong oscillations of airflow around the antennae that temporally structure odor plumes. Behaviors that cause self-induced sensory stimulation often impose selective pressures that influence neural circuit evolution, specifically fostering the emergence of corollary discharge circuits. A candidate corollary discharge circuit in Manduca sexta was previously identified, consisting of a pair of mesothoracic to deutocerebral histaminergic neurons (MDHns), each projecting from the mesothoracic ganglion bilaterally to both antennal lobes (ALs), the first olfactory neuropil. Consistent with a hypothetical role in providing the olfactory system with a corollary discharge, comparative immunohistochemistry reveals that the MDHns innervate the ALs of advanced and basal moths, but not butterflies, which rely more heavily on vision rather than olfaction and have distinctive flight patterns. Characteristic anatomical features of the MDHns reveal that ancestral neurons likely arose in crustaceans and in many species the cells innervate mechanosensory areas of the brain, but not the olfactory system. We therefore propose that the ancestral function of the MDHns was to provide information about self-induced stimulation of the antennae and that the MDHns were exapted to provide this information to the olfactory system in moths. The MDHns therefore represent an example of architectural exaptation, in which identified neurons that originally provided information about motor output to mechanosensory regions have been co-opted to provide information to an additional neural network.

\section{INTRODUCTION}

Exaptation is a core feature in the development of new phenotypic traits, allowing pre-existing traits to be co-opted to take on new or additional roles. There are numerous examples of exaptations involving the co-option of genes $(1,2)$, body appendages $(3,4)$, and behaviors $(5$, 6). For instance, the co-option of the teleost gas bladder into lungs, and lobe fins into limbs supported the conquest of land by tetrapods (7). Feathers are another commonly cited example of exaptation; originally thought to support thermoregulation, they were exapted to produce thrust during flight (8-10). While there are several examples of exaptation in the nervous system in general $(11,12)$ the cellular and neural circuit basis for nervous system exaptation are poorly understood. For example, primitive insect wings originated from tracheal gills present on all body segments (13), yet the specific changes that occurred within motor networks to allow flight motor control in present-day insects remain to be identified. However, as traits such as appendages are co-opted to take on additional functions the neural networks associated with these structures are likely also co-opted for use in this new function.

Subtle cellular and molecular changes to individual neural networks can produce distinct changes in network dynamics and behavioral output. Many evolutionary changes in the nervous system arise from adapting biophysical and synaptic properties to alter network dynamics. This has been most notably demonstrated in networks that produce rhythmic output such as central pattern generators (14-26). In addition to changes in biophysical and synaptic physiology, 
exaptations can also involve changes in neural architecture, such that neurons can be co-opted to innervate neural networks to which they did not project in the ancestral state. Architectural changes have the potential to modify existing brain regions to perform new functions (12). In theory, the exaptation of circuitry could provide an existing network with additional information to enhance processing. Although there is evidence for neural exaptation within the context of entire brain regions (27), very little is known about the potential for neural exaptation at the level of individual neurons. In this study, we used comparative neuroanatomy to describe an example of architectural neural exaptation at the level of a pair of identified neurons. We demonstrate that histaminergic neurons from a motor-to-mechanosensory circuit found throughout the arthropods was co-opted to serve additional olfactory function in moths. This trait was subsequently lost in butterflies in correlation with changes in flight biomechanics and sensory dominance from olfaction to vision for locating food sources. This suggests that co-option of neural circuits at the level of individual neurons can result as a byproduct of behavior-specific natural selection.

\section{METHODS}

\section{Animals}

Insect species used in this study were either collected near Morgantown, WV, reared at WVU from eggs or donated. Manduca sexta were raised and maintained on a standard artificial diet as previously described (28). Bombyx mori were purchased from Mulberry farms (Fallbrook, $\mathrm{CA}$ ), and also raised on standard artificial diet. Idia aemula were collected in Morgantown, WV with the assistance of Dr. John Boback. Pieris rapae and Theatops californiensis were purchased from Carolina Biological Supply Co. (Burlington, NC). Papilio appalachiensis were collected in Morgantown, WV (Coppers Rock State Park). Limenitis archippus were collected in Morgantown, WV. Grapholita molesta were provided by Dr. Mark Willis (Case Western Reserve University). Galleria mellonella, Gyna Lurida, Tenebrio molitor and Oncopeltus fasciatus were provided by Dr. George Keeney (The Ohio State University). Caddisflies from family Limnephilidae were provided by Kathy Kyle Stout (Wildscape Inc.). Drosophila melanogaster were raised on standard medium at $22-25^{\circ} \mathrm{C}$. Amblyomma americanum were provided by Dr. Timothy Driscoll (West Virginia University). At least 6 individuals were used for each species.

\section{Immunocytochemistry}

Histamine labeling was performed as previously described $(29,30)$. Brains and thoracic ganglia were dissected, either separately or connected for experiments. Post-dissection, brains were fixed in a 4\% solution of N-3-dimethylaminopropyl-N'-ethylcarbodiimide (Sigma-Aldrich, 03449) in phosphate buffered saline (PBS, pH 6.9; Sigma-Aldrich, P-5368) at $4^{\circ} \mathrm{C}$ between $2-6$ hours depending on tissue volume (e.g. 2 hours for D. melanogaster, 6 hours for M. sexta). Tissue was then fixed in a 4\% solution of paraformaldehyde (Electron Microscopy Sciences, 15710) in 0.01M PBS overnight. Post-fixation, brains were washed in PBS. For sectioned tissue, brains were embedded in 5\% agarose (Sigma-Aldrich, SLBJ3744V) and sectioned between 100 and $150 \mu \mathrm{m}$ using a Leica VT $1000 \mathrm{~S}$ vibratome. The tissue was washed in $0.5 \%$ Triton $^{\mathrm{TM}}-\mathrm{X} 100$ (PBST; Sigma-Aldrich, 110M0009V), blocked in 2\% bovine serum albumin (BSA; Jackson Laboratory, 001-000-162) for 1 hour. Brains were then incubated in 1:50 mouse anti-bruchpilot (Developmental Studies Hybridoma Bank, nc82) with $2 \%$ BSA in PBST at $4^{\circ} \mathrm{C}$ for 5 days before adding 1:500 Rabbit anti-histamine, and incubating for another two days. The histamine 
antibody was raised against histamine conjugated to succinylated keyhole limpet hemocyanin via carbodiimide and this antibody shows no cross-reactivity keyhole limpet hemocyanin alone (30). Preadsorption with histamine also eliminates labeling (31). Finally, in D. melanogaster, histidine decarboxylase mutants lack histamine immunolabeling using this antibody (32). Following primary antibody application, tissue was washed in PBST, then blocked (as above), and incubated in 1:1000 Alexa 488, or 546(Alexa Flour®; Thermo Fisher Scientific A-11008, A11030). Tissue was washed in PBST and PBS. For sectioned brains, tissue was run through an ascending glycerol (Sigma-Aldrich, BCBN3647V) series (40\%, 60\%, and $80 \%)$ and mounted in Vectashield® (Vector laboratories, Za1222). For whole mounts, tissue was run through an ascending ethanol (Sigma-Aldrich, SHBF6704V) series (30\%, 50\%, 70\%, 95\%, and 100\%) for 10 minutes; tissue was placed in a 1:1 solution of ethanol and methyl salicylate for 15 minutes, then $100 \%$ methyl salicylate for 15 minutes, then mounted in Permount ${ }^{\circledR}$ (Fisher Scientific, SP15-500).

\section{Optical Imaging Acquisition and Analysis}

Fluorescent tissue was viewed with a laser scanning confocal microscope (Olympus FV1000) equipped with red/green $\mathrm{HeNe}$, and argon lasers. Images were acquired using either a $20 \mathrm{x}$ or 40x magnification optical objective. Distance between confocal planes were optimized for the objective (1.79 um for 20X and 0.54 um for 40X) using Fluoview software (FV10-ASW Version 04.00.02.09). Pixel resolution was adjusted to compensate for the size of each specimen between 1024 x 1024 to 2048 × 2048 pixels. Images were only modified for contrast enhancement. Figures were organized in CorelDraw (Version X4).

\section{RESULTS}

In Manduca sexta, the MDHns branch extensively within the mesothoracic ganglion and project ascending axons to innervate the subesophageal zone (SEZ), antennal mechanosensory and motor center (AMMC) and AL $(31,33)$ (Fig., 1A). However, while histaminergic neurons in the MsG of cricket $(34,35)$, locust $(36)$ and Drosophila $(32,37)$ project ascending axons into the AMMC, they do not innervate the AL. This suggests that while the MDHns may be present in many insect taxa, they do not necessarily innervate the olfactory system. Differences in wing beating mechanics across insect species result in different effects on odor plumes $(38,39)$ and in $M$. sexta, the olfactory system is able to track odors pulsed at the wing-beat frequency (40, 41). We therefore hypothesized that MDHn innervation of the AL arose due to selective pressures associated with a need to process odors carried by flight-induced air flow oscillations during plume tracking. We used a comparative approach to determine when over evolutionary time the MDHns began to innervate the $A L$ and if this trait was lost with the evolution of different flight biomechanics.

\section{(a) MDHn Innervation of the AL is Specific to Caddisflies and Moths.}

To determine whether MDHn AL innervation was specific to M. sexta (Sphingidae), we examined the MDHns in Bombyx mori (Bombycidae), a closely related species with similar wing beating frequency and mechanics $(42,43)$. Both moths belong to the superfamily Bombycoidea and $B$. mori, although flightless, must beat their wings while walking to successfully track odor plumes (44). The MDHns have a distinct, consistent morphology that, in combination with HAimmunolabeling allow their identification between species. In M. sexta, MDHn somata were 
located ventrally and send primary neurites dorsally where they project radially throughout the MsG (Fig. 1B) In addition, the MDHns project a single axon that ascends via the ventral nerve cord to the brain. HA-ir was present in the ALs of M.sexta in several ventral glomeruli (Fig. 1C). The MDHns in B. mori possessed nearly identical morphology with ventrally located cell bodies, dorsal radial MsG projections and axons that ascend to the brain (Fig 1D). Similar to M. sexta, $\mathrm{HA}$-ir was present in the $\mathrm{AL}$ of $B$. mori in several ventral glomeruli (Fig. 1E). To determine the phylogenetic distribution of $A L$ innervation by the MDHns in the Macrolepidoptera further, we examined Idia aemula (Erebidae), the powdered snout, which belongs to the superfamily Noctuoidea. The MsG of $I$. aemula contains histaminergic neurons with nearly identical morphology to the MDHns in M. sexta and B. mori, (Fig. 1F), including ascending projections to the brain and bilateral innervation of both ALs (Fig. 1G). These results together indicate that histaminergic neurons projecting from flight motor centers to the olfactory system are conserved within macroplepidopteran moths.

Butterflies also belong to the Macrolepidoptera, but primarily use vision to locate mates and food (45). The flight patterns of butterflies are also much more heterogeneous than moths due to non-periodic wing flapping, gliding, and turn unpredictability (46). These characteristics lower predation risk (46), but would reduce plume tracking ability. Butterflies are relatively closely related to the Bombycoidea and thus make great candidates for studying the emergence of MDHn innervation of the AL. Due to these differences between butterfly and moth flight behavior, we hypothesized that diurnal, and visually guided butterflies would have no AL MDHn innervation. We examined the ALs and MsGs of representative species from three of the five total families of butterflies (Nymphalidae, Papilionidae, and Pieridae). In Pieris rapae (Pieridae), ventrally located MDHns in the MsG project ascending axons along the ventral nerve cord to the brain, and have a general architecture similar to $M$. sexta (Fig. 2A \& B). However, in $P$. rapae there were no HA-ir processes detected in the ALs (Fig 2C). The ALs of Limenitis archippus (Nymphalidae) also lack HA-ir processes (Figure 2D). Finally, the MDHns of Papilio appalachiensis (Papilionidae) also branch radially throughout the MsG and project to the brain via the ventral nerve cord, but again HA-ir processes were absent within the AL (Fig 2E \& F). These results together suggest that MDHn innervation of the AL was either lost in butterflies or arose in the macrolepidopteran moths.

The Microlepidoptera are the most basal lepidopterans and are therefore ideally situated to determine if AL HA innervation by the MDHns had been lost in butterflies, or arose in the macroplepidopteran moths. To this end, we examined the MDHns of two microlepidopterans, Grapholita molesta (Tortricidae) and Galleria mellonella (Pyralidae), both of which walk and fan their wings during their final approach to an odor source $(47,48)$. Similar to $B$. mori, the MDHn axons of $G$. molesta ascend from the MsG (Fig. 3A) via the ventral nerve cord to innervate the ALs (Fig. 3B). This was also the case for G. mellonella (Pyralidae) (Fig. 3C \& D). We next examined the MDHns of one species of caddisfly (Limnephilidae) as the Trichoptera are the closest related order to the Lepidoptera. Similar to moths, the MDHns of caddisflies have ventrally located cell bodies that project ascending fibers to the brain (Figure 3E) that innervate the ALs (Fig. 3F) These results suggest that MDHn innervation of the ALs was present in a common ancestor of the Lepidoptera and caddisflies, but subsequently lost in the butterflies.

\section{(b) MDHns are Present Throughout the Arthropods}

The olfactory systems of many arthropods species, including insects, are innervated by HA-ir processes from sources other than MDHns $(30,49-54)$, while the olfactory systems of other 
species lack HA-ir altogether $(29,30,32,55)$. To determine when the MDHns arose, we performed HA immunocytochemistry on the MsG of arthropods. Drosophila melanogaster (Drosophilidae) possess MDHns with the characteristic radial planar projections within the MsG and ascending projections (Fig. 4A). However, while these ascending projections innervate the SEZ and AMMC, they do not enter the ALs (Figure 4B). In Tenebrio molitor (Coleoptera), Oncopeltus fasciatus (Hemiptera), and Gyna lurida (Blattodea), ventrally located cell bodies with ascending $\mathrm{HA}$ fibers were also observed in the MsG (Figure 4C, D \& E), as is also the case for the maxillulary cephalic neuromere of the copepod Calanus finmarchicus (Crustaceae; (51)) and in the thoracic ganglia of the lobster Homarus americanus (Crustaceae; (56)). In the centipede Theatops californiensis, at least three pairs of histaminergic neurons were located along the midline of ganglia within segments bearing the second pair of legs (Fig. 4F), the equivalent to the mesothoracic ganglion in insects. One pair of midline cells possessed ventral cell bodies and ascending axons. The extent of branching of these cells within the ganglion was minimal, but the axons were located dorsally, consistent with all other species observed. Finally, in the tick Amblyomma americanum (Chelicerata; Ixodidae), dorsally and laterally located cell bodies were observed, and there were no ascending projections (Figure 4G), rather these cells projected diffusely in most neuromeres of the synganglion. In particular, we observed dense histaminergic innervation of the pedal, and cheliceral neuromeres, areas that process leg and mouthpart sensorimotor information (57). This distribution of histaminergic neurons was similar to that observed in the synganglia of spiders $(58,59)$. These neurons seem unlikely to be homologs of the MDHns as their cell bodies are dorsally located and reside along the lateral margin of the synganglion. Thus, MDHns appear to be widely distributed within the arthropods, and while homologous neurons are not apparent in ticks, histaminergic neurons that interconnect limb control and somatosensory regions appear to be a common feature of the arthropod nervous system.

\section{DISCUSSION}

Using a comparative approach to study specific neural circuits provides insight into how circuits are co-opted to perform new functions within a relatively short evolutionary time. Selective pressures drive neural circuit evolution. Here, we hypothesized that the presence of a circuit interconnecting the flight motor and olfactory systems would depend on the influence of flight mechanics that arose among nocturnal plume tracking insects and the impact that these mechanics have on the sensory field of the insect. In this study we found that a morphologically distinct neuron that ascends from the MsG to innervate the AL arose after the last common ancestor of the Diptera and Lepidoptera. This circuit was conserved across much of the Lepidoptera, however this trait was lost in butterflies which differ dramatically from moths in their behavioral ecology. Furthermore, paired, histaminergic neurons that ascend from motor centers in the ventral nerve cord to the brain appear to be conserved within the insects and crustaceans. However, in ticks (Fig. 4) and spiders $(58,59)$ the palpal/pedipalpal ganglia receive dense innervation from $\mathrm{HA}$-ir neurons with dorso-laterally located somata, suggesting that the MDHns (which have ventromedial somata) likely arose after the Chelicerates. Regardless of origin, across all arthropods in this study and others, there exist histaminergic neurons that interconnect ganglia representing different body segments.

Why would specific groups of insects use flight information during olfactory guided behaviors, but not others? Wing beating creates air turbulence, thus affecting the structure of odor plumes. 
In M. sexta, wing beating in tethered flight creates strong oscillatory flow of air over the antennae that is tightly coupled to the wing beat frequency (39). Moths use odor plumes to locate mates, food, and oviposition sites and the mechanics of wing-beating in $B$. mori while walking (42) and $M$. sexta while hovering (43) are similar with respect to frequency, and wing tip pattern, suggesting similar impacts on odor plumes. These flight-induced oscillations in airflow therefore create a periodic temporal structure of ecologically important stimuli. Butterflies, however, have strikingly different flight mechanics from moths. While moths have a consistent wing beat frequency and stroke during odor-guided flight $(43,60,61)$, butterflies have a much more stochastic wing beat, and flight path (46). Indeed, many butterflies incorporate protean behavior into their flight patterns which ultimately creates a flight pattern with wingbeat frequencies that aren't as stable as in moths, which may be a means to avoid predation (46). Furthermore, although male and female butterflies produce pheromones, they are used as short-range cues (1-2 meters) to determine mate quality $(45,62)$, whereas male moths can track female pheromones over distances of several tens of meters (63). Although the path of the wing tip during each wing stroke is similar between $D$. melanogaster and moths (64), the wing beat frequency of $D$. melanogaster is $\sim 190-230 \mathrm{~Hz}(65)$, which much higher than the $\sim 27-28 \mathrm{~Hz}$ wing beat frequency of $M$. sexta (39). Furthermore, antennal responses across several insect species can track rapid odor concentration fluctuations $(40,66,67)$, depending on species perhaps exceeding $100 \mathrm{~Hz}$ (68). Local field potentials within the AL have also been shown to respond to fluctuations at least up to $\sim 70 \mathrm{~Hz}(40)$, well within the range of Lepidopteran wing beat frequencies. In addition, neural population responses from the AL of $M$. sexta track and represent olfactory stimuli optimally when odors are presented at the wingbeat frequency (41). This finding also corresponds to enhanced olfactory acuity as measured behaviorally (69) supporting the conclusion that their olfactory system has adapted to encode information that is embedded within a temporal structure induced by their own active sampling behavior. The disturbances caused by the very high frequency wing-beating in Drosophila on the other hand, are unlikely to be tracked by the AL, while the lower frequency disturbances induced by $M$. sexta wing beating alter the structure of odor plumes in a manner that affects odor evoked activity in the AL.

Typically, across more moderate periods of evolutionary time, neural circuits change by dedicating more space and resources to processing stimuli that are most important for an ecological niche. For instance, roughly a third of the antennal lobe of male $M$. sexta is devoted to processing female sex pheromone (70-73) and cortical expansion in star-nosed moles, hedgehogs, and moles reflect species-specific changes in ecological niches and sensory appendages (74). While many examples exist of the expansion and reduction of brain areas over time, very few examples exist of the invasion of new brain regions by identified neurons that are conserved across a broad range of species. Rather than an expansion within the context of a pre-existing function, the innervation of the ALs by the MDHns represent an example of co-option of a circuit into an additional network that serves a different function. The appearance, and subsequent loss of MDHns innervation of the ALs suggests that individual neurons can be co-opted to provide input to neural networks that they do not influence in other species and that large changes in neural circuit architecture can be gained and lost in a relatively short period of time.

We observed ascending histaminergic neurons that innervated mechanosensory regions for head appendages in the brains of arthropods that span $\sim 250$ million years of evolution. In $D$. melanogaster, as well as all moths and butterflies, MDHns innervate the AMMC, and even in 
ticks, which may lack MDHn homologs, there was dense histaminergic innervation of the dorsal anterior portion of the synganglion which receives sensory input from the mouthparts (57). The conservation of this trait suggests that information about limb motor output is a critical component of mechanosensory network activity. The presence of interganglionic histaminergic neurons in the AMMC could also reflect the co-option of head appendages themselves from a locomotory function, to mechanosensory, and then olfactory function $(75,76)$. Our data suggest that behavioral and morphological specializations in moths resulted in the co-option of this circuit that provides input to a mechanosensory network in the ancestral state to also provide additional input to the olfactory system.

Acknowledgments: We would like to thank John Boback, Mark Willis, George Keeney, Kathy Kyle Stout and Timothy Driscoll for generously providing various arthropod species. We would also like to thank for helpful comments on earlier versions of this manuscript.

Funding: This work was funded by National Institutes of Health Grants 1 R03 DC013997-01 to AMD and USAFOSR FA9550-17-1-0117 to KCD and AMD, as well as WVU Center for Neuroscience Summer Undergraduate Research Internships (NIGMS 5P30 GM103503) to $\mathrm{MMH}$, EJH and KER.

\section{Works Cited}

1. True JR, Carroll SB. Gene co-option in physiological and morphological evolution. Annu Rev Cell Dev Bi. 2002;18:53-80. PubMed PMID: WOS:000179413400004. English.

2. Harlin-Cognato A, Hoffman EA, Jones AG. Gene cooption without duplication during the evolution of a male-pregnancy gene in pipefish. P Natl Acad Sci USA. 2006 Dec 19;103(51):19407-12. PubMed PMID: WOS:000243166600035. English.

3. Clack JA. The emergence of early tetrapods. Palaeogeogr Palaeocl. 2006 Mar 22;232(24):167-89. PubMed PMID: WOS:000236519100006. English.

4. Shubin N, Tabin C, Carroll S. Deep homology and the origins of evolutionary novelty. Nature. 2009 Feb 12;457(7231):818-23. PubMed PMID: WOS:000263266700031. English.

5. Borgia G, Coleman SW. Co-option of male courtship signals from aggressive display in bowerbirds. Proc Biol Sci. 2000 Sep 07;267(1454):1735-40. PubMed PMID: 12233770. Pubmed Central PMCID: 1690730.

6. Weible D. Ritualization and Exaptation: Towards a Theory of Hierarchical Contextuality? Biosemiotics-Neth. 2012 Aug;5(2):211-26. PubMed PMID: WOS:000305561300006. English.

7. Ahlberg PE, Clack JA. Palaeontology: a firm step from water to land. Nature. 2006 Apr 06;440(7085):747-9. PubMed PMID: 16598240.

8. Ostrom JH. Archaeopteryx and Origin of Flight. Q Rev Biol. 1974;49(1):27-47. PubMed PMID: WOS:A1974T039800002. English.

9. Ostrom JH. Bird Flight - How Did It Begin. Am Sci. 1979;67(1):46-56. PubMed PMID: WOS:A1979GG07000012. English.

10. Bakker RT. Dinosaur Renaissance. Scientific American. 1975;232(4):58-\&. PubMed PMID: WOS:A1975V971100004. English.

11. Mcgurk H, Macdonald J. Hearing Lips and Seeing Voices. Nature. 1976;264(5588):7468. PubMed PMID: WOS:A1976CQ45400031. English.

12. Anderson ML. Neural reuse: A fundamental organizational principle of the brain. Behavioral and Brain Sciences. 2010 Aug;33(4):245-+. PubMed PMID: WOS:000284381100001. English. 
13. Dumont JPC, Robertson RM. Neuronal Circuits - an Evolutionary Perspective. Science. 1986 Aug 22;233(4766):849-53. PubMed PMID: WOS:A1986D609100017. English.

14. Dickinson PS. Neuromodulation of central pattern generators in invertebrates and vertebrates. Curr Opin Neurobiol. 2006 Dec;16(6):604-14. PubMed PMID: 17085040.

15. Katz PS, Frost WN. Intrinsic neuromodulation in the Tritonia swim CPG: the serotonergic dorsal swim interneurons act presynaptically to enhance transmitter release from interneuron C2. J Neurosci. 1995 Sep;15(9):6035-45. PubMed PMID: 7666187. Epub 1995/09/01. eng.

16. Newcomb JM, Katz PS. Different functions for homologous serotonergic interneurons and serotonin in species-specific rhythmic behaviours. Proc Biol Sci. 2009 Jan 7;276(1654):99108. PubMed PMID: 18782747. Epub 2008/09/11. eng.

17. Newcomb JM, Sakurai A, Lillvis JL, Gunaratne CA, Katz PS. In the Light of Evolution VI: Brain and Behavior: Homology and homoplasy of swimming behaviors and neural circuits in the Nudipleura (Mollusca, Gastropoda, Opisthobranchia). Proc Natl Acad Sci U S A. 2012 Jun 20. PubMed PMID: 22723353. Epub 2012/06/23. Eng.

18. Newcomb JM, Sakurai A, Lillvis JL, Gunaratne CA, Katz PS. Homology and homoplasy of swimming behaviors and neural circuits in the Nudipleura (Mollusca, Gastropoda, Opisthobranchia). Proc Natl Acad Sci U S A. 2012 Jun 26;109 Suppl 1:10669-76. PubMed PMID: 22723353. Epub 2012/06/23. eng.

19. Harris-Warrick RM. Neuromodulation and flexibility in Central Pattern Generator networks. Current Opinion in Neurobiology. 2011 Oct;21(5):685-92. PubMed PMID: WOS:000298121400005. English.

20. Katz PS, Harris-Warrick RM. The evolution of neuronal circuits underlying speciesspecific behavior. Curr Opin Neurobiol. 1999 Oct;9(5):628-33. PubMed PMID: 10508740. Epub 1999/10/06. eng.

21. Sakurai A, Newcomb JM, Lillvis JL, Katz PS. Different roles for homologous interneurons in species exhibiting similar rhythmic behaviors. Curr Biol. 2011 Jun 21;21(12):1036-43.

PubMed PMID: 21620707. Epub 2011/05/31. eng.

22. Birmingham JT, Tauck DL. Neuromodulation in invertebrate sensory systems: from biophysics to behavior. J Exp Biol. 2003 Oct;206(Pt 20):3541-6. PubMed PMID: 12966045.

23. Nusbaum MP, Beenhakker MP. A small-systems approach to motor pattern generation. Nature. 2002 May 16;417(6886):343-50. PubMed PMID: WOS:000175592100059. English.

24. Briggman KL, Kristan WB. Multifunctional pattern-generating circuits. Annu Rev Neurosci. 2008;31:271-94. PubMed PMID: 18558856. Epub 2008/06/19. eng.

25. Marder E, Calabrese RL. Principles of rhythmic motor pattern generation. Physiol Rev. 1996 Jul;76(3):687-717. PubMed PMID: 8757786. Epub 1996/07/01. eng.

26. Bargmann $\mathrm{Cl}$. Beyond the connectome: how neuromodulators shape neural circuits.

Bioessays. 2012 Jun;34(6):458-65. PubMed PMID: 22396302.

27. Anderson ML. Evolution of cognitive function via redeployment of brain areas.

Neuroscientist. 2007 Feb;13(1):13-21. PubMed PMID: 17229971.

28. Bell RA, Joachim FG. Techniques for Rearing Laboratory Colonies of Tobacco Hornworms and Pink Bollworms Lepidoptera-Sphingidae-Gelechiidae. Ann Entomol Soc Am. 1976;69(2):365-73. PubMed PMID: WOS:A1976BJ88500035. English.

29. Dacks AM, Nighorn AJ. The organization of the antennal lobe correlates not only with phylogenetic relationship, but also life history: a Basal hymenopteran as exemplar. Chem Senses. 2011 Jan;36(2):209-20. PubMed PMID: 21059697. Pubmed Central PMCID: 3105589. Epub 2010/11/10.

30. Dacks AM, Reisenman CE, Paulk AC, Nighorn AJ. Histamine-immunoreactive local neurons in the antennal lobes of the hymenoptera. J Comp Neurol. 2010 Aug 1;518(15):291733. PubMed PMID: 20533353. Pubmed Central PMCID: 2886666. Epub 2010/06/10. eng. 
31. Bradley SP, Chapman PD, Lizbinski KM, Daly KC, Dacks AM. A Flight Sensory-Motor to Olfactory Processing Circuit in the Moth Manduca sexta. Front Neural Circuits. 2016;10:5. PubMed PMID: 26909026. Pubmed Central PMCID: 4754697.

32. Melzig J, Buchner S, Wiebel F, Wolf R, Burg M, Pak WL, et al. Genetic depletion of histamine from the nervous system of Drosophila eliminates specific visual and mechanosensory behavior. Journal of Comparative Physiology a-Sensory Neural and Behavioral Physiology. 1996 Dec;179(6):763-73. PubMed PMID: WOS:A1996VW45500004. English.

33. Homberg U, Hildebrand JG. Histamine-immunoreactive neurons in the midbrain and suboesophageal ganglion of sphinx moth Manduca sexta. J Comp Neurol. 1991 May 22;307(4):647-57. PubMed PMID: 1869635. Epub 1991/05/22. eng.

34. Horner M. Cytoarchitecture of histamine-, dopamine-, serotonin- and octopaminecontaining neurons in the cricket ventral nerve cord. Microsc Res Techniq. 1999 Feb 1;44(23):137-65. PubMed PMID: WOS:000078121100007. English.

35. Horner M, Helle J, Schurmann FW. The distribution of histamine-immunoreactive neurons in the ventral nerve cord of the cricket, Gryllus bimaculatus. Cell and Tissue Research. 1996 Dec;286(3):393-405. PubMed PMID: WOS:A1996WG13100012. English.

36. Patschke A, Bicker G. Development of histamine-immunoreactivity in the Central nervous system of the two locust species Schistocerca gregaria and Locusta migratoria. Microsc Res Tech. 2011 Oct;74(10):946-56. PubMed PMID: 21484940.

37. Liu WW, Wilson RI. Glutamate is an inhibitory neurotransmitter in the Drosophila olfactory system. Proc Natl Acad Sci U S A. 2013 Jun 18;110(25):10294-9. PubMed PMID: 23729809. Pubmed Central PMCID: 3690841.

38. Gomez-Marin A, Duistermars BJ, Frye MA, Louis M. Mechanisms of odor-tracking: multiple sensors for enhanced perception and behavior. Front Cell Neurosci. 2010;4:6. PubMed PMID: 20407585. Pubmed Central PMCID: 2854573.

39. Sane SP, Jacobson NP. Induced airflow in flying insects II. Measurement of induced flow. J Exp Biol. 2006 Jan;209(Pt 1):43-56. PubMed PMID: 16354777. Epub 2005/12/16. 40. Tripathy SJ, Peters OJ, Staudacher EM, Kalwar FR, Hatfield MN, Daly KC. Odors Pulsed at Wing Beat Frequencies are Tracked by Primary Olfactory Networks and Enhance Odor Detection. Front Cell Neurosci. 2010;4:1. PubMed PMID: 20407584. Pubmed Central PMCID: 2854572. Epub 2010/04/22.

41. Houot B, Burkland R, Tripathy S, Daly KC. Antennal lobe representations are optimized when olfactory stimuli are periodically structured to simulate natural wing beat effects. Front Cell Neurosci. 2014;8:159. PubMed PMID: 24971052. Pubmed Central PMCID: 4053783.

42. Mishima T, Kanzaki R. Coordination of flipflopping neural signals and head turning during pheromone-mediated walking in a male silkworm moth Bombyx mori. Journal of Comparative Physiology a-Sensory Neural and Behavioral Physiology. 1998 Sep;183(3):27382. PubMed PMID: WOS:000076277700001. English.

43. Willmott AP, Ellington CP. The mechanics of flight in the hawkmoth Manduca sexta. I. Kinematics of hovering and forward flight. J Exp Biol. 1997 Nov;200(Pt 21):2705-22. PubMed PMID: 9418029.

44. Obara Y. Bombyx-Mori Mating Dance - Essential in Locating the Female. Appl Entomol Zool. 1979;14(1):130-2. PubMed PMID: WOS:A1979GQ22600019. English.

45. Scott JA. Mate-Locating Behavior of Butterflies. Am Midl Nat. 1974;91(1):103-17. PubMed PMID: WOS:A1974S186900004. English.

46. Srygley RB, Chai P. Flight Morphology of Neotropical Butterflies - Palatability and Distribution of Mass to the Thorax and Abdomen. Oecologia. 1990;84(4):491-9. PubMed PMID: WOS:A1990EF11400009. English. 
47. Giner M, Balcells M, Avilla J. Insecticidal action of five allyl esters on eggs and larvae of three tortricid fruit pests: laboratory tests. B Insectol. 2012 Jun;65(1):63-70. PubMed PMID: WOS:000304223700009. English.

48. Roller H, Biemann K, Bjerke JS, Norgard DW, Mcshan WH. Sex Pheromones of Pyralid Moths .I. Isolation and Identification of Sex-Attractant of Galleria Mellonella $L$ (Greater Waxmoth). Acta Entomol Bohemos. 1968;65(3):208-\&. PubMed PMID: WOS:A1968B729200006. English.

49. Bornhauser BC, Meyer EP. Histamine-like immunoreactivity in the visual system and brain of an orthopteran and a hymenopteran insect. Cell Tissue Res. 1997 Jan;287(1):211-21. PubMed PMID: 9011397.

50. Gebhardt S, Homberg U. Immunocytochemistry of histamine in the brain of the locust Schistocerca gregaria. Cell Tissue Res. 2004 Aug;317(2):195-205. PubMed PMID: 15221442. 51. Hartline DK, Christie AE. Immunohistochemical mapping of histamine, dopamine, and serotonin in the central nervous system of the copepod Calanus finmarchicus (Crustacea; Maxillopoda; Copepoda). Cell Tissue Res. 2010 Jul;341(1):49-71. PubMed PMID: 20532915. 52. Ignell R. Monoamines and neuropeptides in antennal lobe interneurons of the desert locust, Schistocerca gregana: an immunocytochemical study. Cell Tissue Res. 2001 Oct;306(1):143-56. PubMed PMID: 11683175.

53. Loesel R, Homberg U. Histamine-immunoreactive neurons in the brain of the cockroach Leucophaea maderae. Brain Res. 1999 Sep 25;842(2):408-18. PubMed PMID: 10526137. Epub 1999/10/20. eng.

54. Callaway JC, Stuart AE. The distribution of histamine and serotonin in the barnacle's nervous system. Microsc Res Tech. 1999 Jan 15-Feb 1;44(2-3):94-104. PubMed PMID: 10084829.

55. Liu WW, Wilson RI. Transient and specific inactivation of Drosophila neurons in vivo using a native ligand-gated ion channel. Curr Biol. 2013 Jul 8;23(13):1202-8. PubMed PMID: 23770187. Pubmed Central PMCID: 3725270.

56. Mulloney B, Hall WM. Neurons with Histamine-Like Immunoreactivity in the Segmental and Stomatogastric Nervous Systems of the Crayfish Pacifastacus-Leniusculus and the Lobster Homarus-Americanus. Cell and Tissue Research. 1991 Oct;266(1):197-207. PubMed PMID: WOS:A1991GH78300021. English.

57. Hummel NA, Li AY, Witt CM. Serotonin-like immunoreactivity in the central nervous system of two ixodid tick species. Exp Appl Acarol. 2007 Dec;43(4):265-78. PubMed PMID: WOS:000251409100003. English.

58. Schmid A, Becherer C. Distribution of histamine in the CNS of different spiders. Microsc Res Tech. 1999 Jan 15-Feb 1;44(2-3):81-93. PubMed PMID: 10084828.

59. Schmid A, Duncker M. Histamine Immunoreactivity in the Central-Nervous-System of the Spider Cupiennius-Salei. Cell and Tissue Research. 1993 Sep;273(3):533-45. PubMed PMID: WOS:A1993LV34700013. English.

60. Liu H, Ellington $\mathrm{C}$, Kawachi K. A computational fluid dynamic study of hawkmoth hovering. J Exp Biol. 1998 Feb;201 (Pt 4):461-77. PubMed PMID: 9438823.

61. Usherwood JR, Ellington CP. The aerodynamics of revolving wings I. Model hawkmoth wings. J Exp Biol. 2002 Jun;205(Pt 11):1547-64. PubMed PMID: 12000800.

62. Andersson J, Borg-Karlson AK, Vongvanich N, Wiklund C. Male sex pheromone release and female mate choice in a butterfly. Journal of Experimental Biology. 2007 Mar 15;210(6):964-70. PubMed PMID: WOS:000244633500010. English.

63. Elkinton JS, Schal C, Ono T, Carde RT. Pheromone Puff Trajectory and Upwind Flight of Male Gypsy Moths in a Forest. Physiol Entomol. 1987 Dec;12(4):399-406. PubMed PMID: WOS:A1987L188000005. English. 
64. Zanker JM. How Does Lateral Abdomen Deflection Contribute to Flight Control of Drosophila-Melanogaster. Journal of Comparative Physiology a-Sensory Neural and Behavioral Physiology. 1988;162(5):581-8. PubMed PMID: WOS:A1988M726200003. English.

65. Lehmann FO, Dickinson $\mathrm{MH}$. The changes in power requirements and muscle efficiency during elevated force production in the fruit fly Drosophila melanogaster. J Exp Biol. 1997 Apr;200(Pt 7):1133-43. PubMed PMID: 9131808.

66. Lemon W, Getz W. Temporal resolution of general odor pulses by olfactory sensory neurons in American cockroaches. J Exp Biol. 1997;200(Pt 12):1809-19. PubMed PMID: 9319720.

67. Bau J, Justus KA, Carde RT. Antennal resolution of pulsed pheromone plumes in three moth species. J Insect Physiol. 2002 Apr;48(4):433-42. PubMed PMID: 12770092.

68. Szyszka P, Gerkin RC, Galizia CG, Smith BH. High-speed odor transduction and pulse tracking by insect olfactory receptor neurons. P Natl Acad Sci USA. 2014 Nov 25;111(47):16925-30. PubMed PMID: WOS:000345662700065. English.

69. Daly KC, Kalwar F, Hatfield M, Staudacher E, Bradley SP. Odor detection in Manduca sexta is optimized when odor stimuli are pulsed at a frequency matching the wing beat during flight. PLoS One. 2013;8(11):e81863. PubMed PMID: 24278463. Pubmed Central PMCID: 3836951.

70. Schneiderman AM, Hildebrand JG, Brennan MM, Tumlinson JH. Trans-sexually grafted antennae alter pheromone-directed behaviour in a moth. Nature. 1986 Oct 30-Nov 5;323(6091):801-3. PubMed PMID: 3774007. Epub 1986/10/05. eng.

71. Christensen TA, Hildebrand JG. Male-specific, sex pheromone-selective projection neurons in the antennal lobes of the moth Manduca sexta. J Comp Physiol A. 1987 May;160(5):553-69. PubMed PMID: 3612589. Epub 1987/05/01.

72. Rospars JP, Hildebrand JG. Sexually dimorphic and isomorphic glomeruli in the antennal lobes of the sphinx moth Manduca sexta. Chem Senses. 2000 Apr;25(2):119-29. PubMed PMID: 10781018. Epub 2000/04/26. eng.

73. Hansson BS, Christensen TA, Hildebrand JG. Functionally distinct subdivisions of the macroglomerular complex in the antennal lobe of the male sphinx moth Manduca sexta. J Comp Neurol. 1991 Oct 8;312(2):264-78. PubMed PMID: 1748732. Epub 1991/10/08. eng.

74. Catania KC. Cortical organization in insectivora: the parallel evolution of the sensory periphery and the brain. Brain Behav Evol. 2000 Jun;55(6):311-21. PubMed PMID: 10971016.

75. Hansson BS, Stensmyr MC. Evolution of insect olfaction. Neuron. $2011 \mathrm{Dec}$ 08;72(5):698-711. PubMed PMID: 22153368.

76. Jockusch EL, Williams TA, Nagy LM. The evolution of patterning of serially homologous appendages in insects. Dev Genes Evol. 2004 Jul;214(7):324-38. PubMed PMID:

WOS:000222644200002. English.

Figure Legends

\section{Figure 1}

The MDHns in Macrolepidopteran Moths Innervate the ALs. A: Schematic of MDHns (green) in Manduca sexta. Each MDHn innervates the SEZ and AMMC before projecting to both ALs B: Whole mount Manduca sexta pterothoracic ganglion immunolabeled for HA. MDHns are the most anterior pair of histaminergic neurons (arrow heads). C: Frontal section of Manduca sexta AL immunolabeled for HA (green). Bruchpilot immunolabeling delineates neuropil (magenta). D: Sagittal section of Bombyx mori MsG immunolabeled for HA. E: Sagittal section of Bombyx mori AL immunolabeled for HA (green). Bruchpilot immunolabeling delineates neuropil (magenta). F: Whole mount sagittal view of Idia aemula pterothoracic ganglion. G. Sagittal section of Idia aemula AL immunolabeled for HA (green). Bruchpilot immunolabeling delineates neuropil 
(magenta). Bruchpilot (magenta) is used to delineate neuropil. HA-ir (green). Scale bars = $100 \mu \mathrm{m}$

\section{Figure 2}

MDHns do not Innervate ALs in Butterflies. A: Full CNS montage of HA-ir in Pieris rapae. B: Whole mount sagittal view of Pieris rapae MsG. C: Pieris rapae AL showing absence of HA-ir (green). Bruchpilot immunolabeling delineates neuropil (magenta). D: Whole mount frontal view of Limenitis archippus of brain showing no AL HA-ir. HA-ir can be seen directly posterior of the left $A L$ in $D$, however optical stacks restricted to the depth of tissue including only the $A L$ demonstrate that these HA-ir processes do not enter the AL. E: Horizontal view of MsG of Papilio appalachiensis. F: Horizontal section of Papilio appalachiensis brain showing no HA-ir (green) in the AL. Bruchpilot (magenta) delineates neuropil. Scale bars $=100 \mu \mathrm{m}$.

\section{Figure 3}

The MDHns in Microlepidoptera and Trichoptera innervate the ALs. A: Whole mount horizontal view of Galleria mellonella MsG. B: Whole mount frontal view of Galleria mellonella brain showing HA-ir (green) in the AL. Bruchpilot (magenta) delineates neuropil. C: Whole mount horizontal view of Grapholita molesta MsG. D: Whole mount frontal view of Grapholita molesta brain. E: Whole mount horizontal view of caddisfly (Limnephilidae) MsG. F: Whole mount frontal view of caddisfly (Limnephilidae) brain showing HA-ir (green) processes within the AL (brackets). Bruchpilot (magenta) delineates neuropil. Scale bars $=100 \mu \mathrm{m}$.

\section{Figure 4}

MDHns are Present in the Majority of Arthropods. A: Whole mount horizontal view of the thoracic ganglia of Drosophila melanogaster. B: Whole mount frontal view of the brain of Drosophila melanogaster. No HA-ir is observed in the ALs (insets). Bruchpilot (magenta) delineates neuropil. C: Whole mount horizontal view of the MsG of Tenebrio molitor immunolabeled for HA. D: Whole mount horizontal view of thoracic ganglia of Oncopeltus fasciatus immunolabeled for HA. E: Whole mount horizontal view of the MsG of Gyna lurida immunolabeled for HA. F: Whole mount horizontal view of the first post-cephalic ganglion in Theatops californiensis immunolabeled for HA. G: Whole mount horizontal view of the synganglion in Amblyomma americanum. Scale bars $=100 \mu \mathrm{m}$.

\section{Figure 5}

Schematic representation of the proposed evolutionary history of the MDHns. In this representation the MDHns originally projected from the mesothoracic ganglion (MsG; blue) to the sub-esophageal zone and antennal mechanosensory and motor center (AMMC/SEZ; lavender). In the last common ancestor of the Lepidoptera and Trichoptera, the MDHns were co-opted (1; Dashed MDHn branches) to innervate the antennal lobes (ALs; magenta). The innervation of the ALs was lost in the butterflies (2), but maintained in macrolepidoteran moths. 
Figure 1:
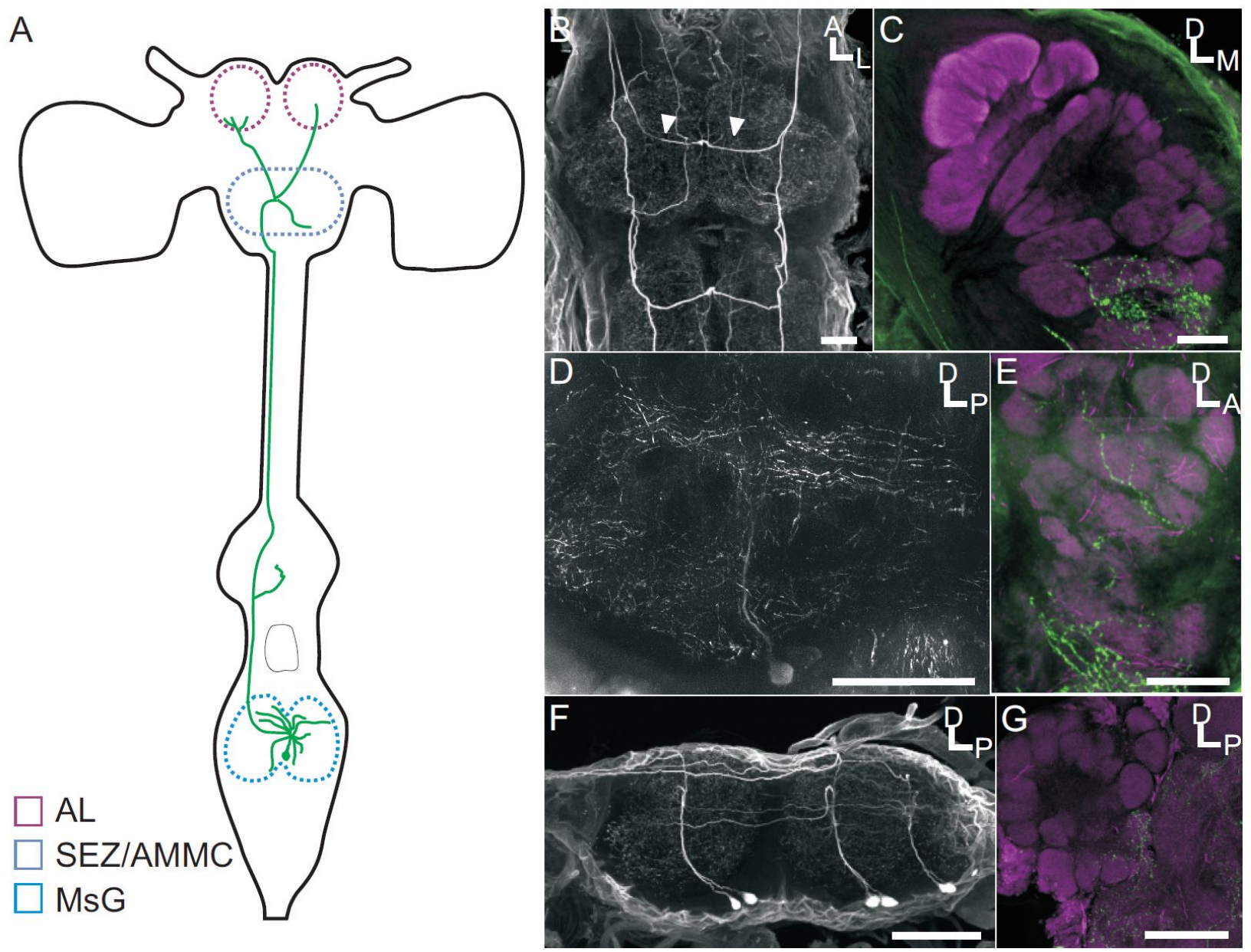
Figure 2:

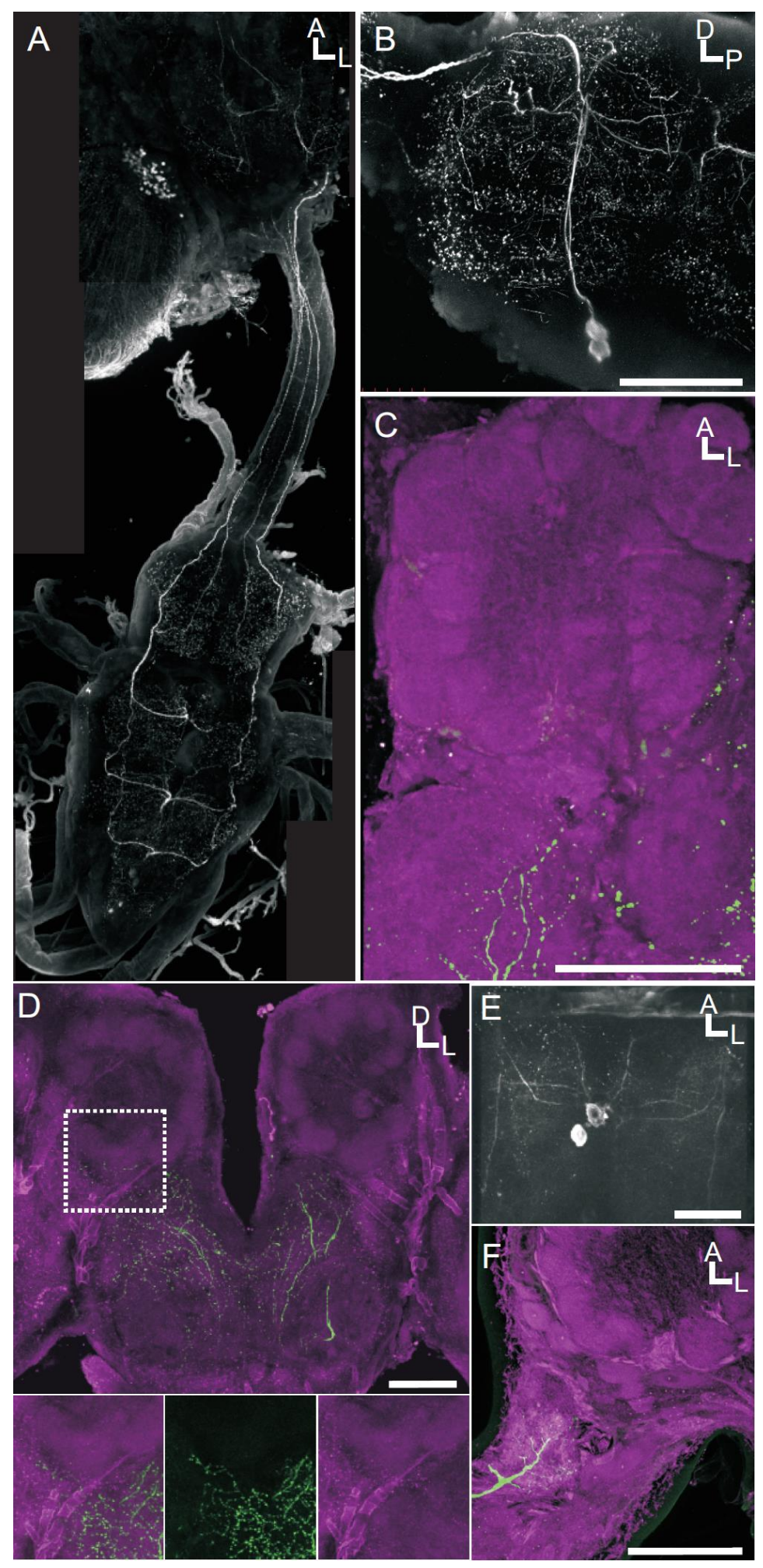


Figure 3:

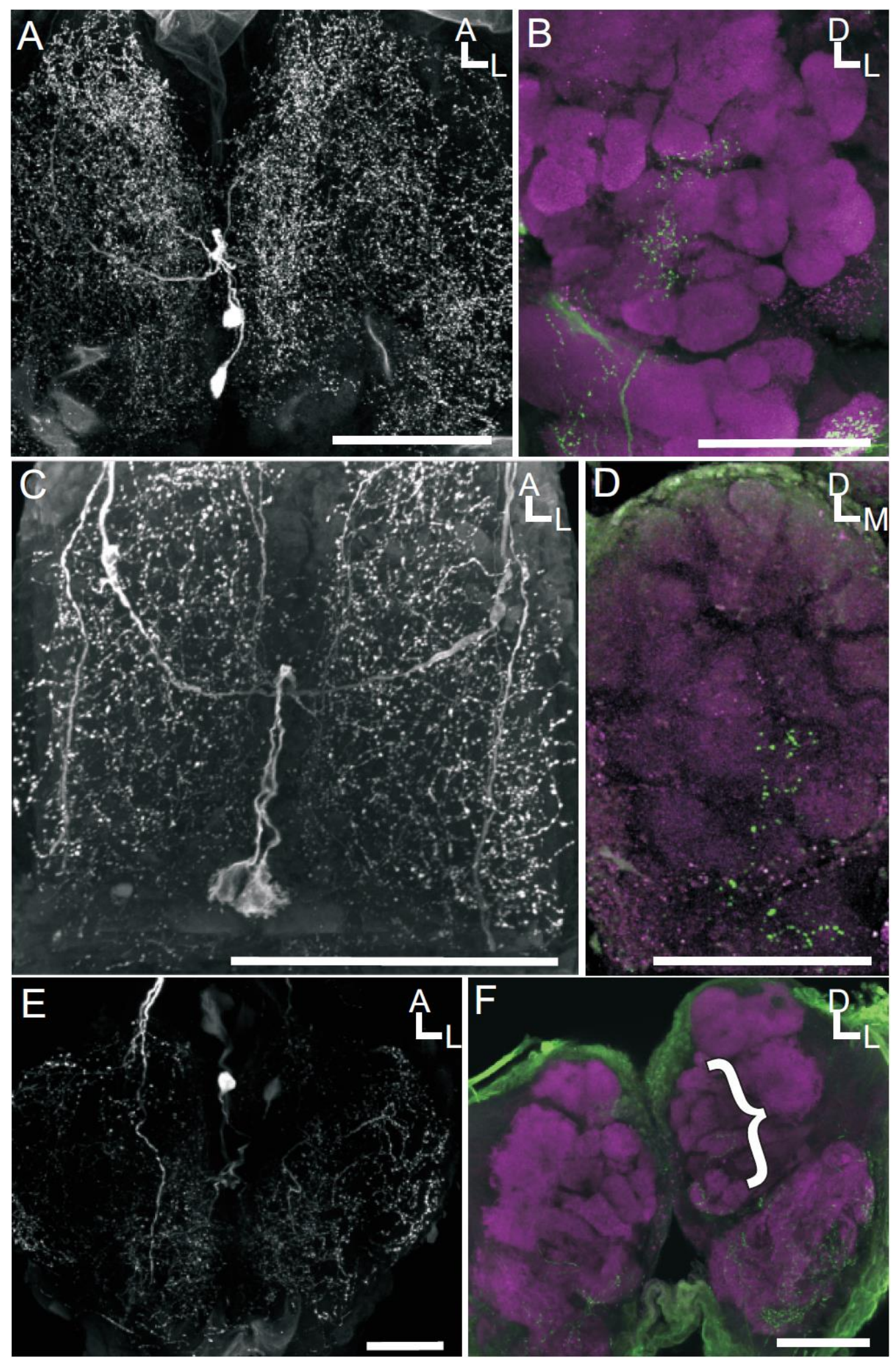


Figure 4:

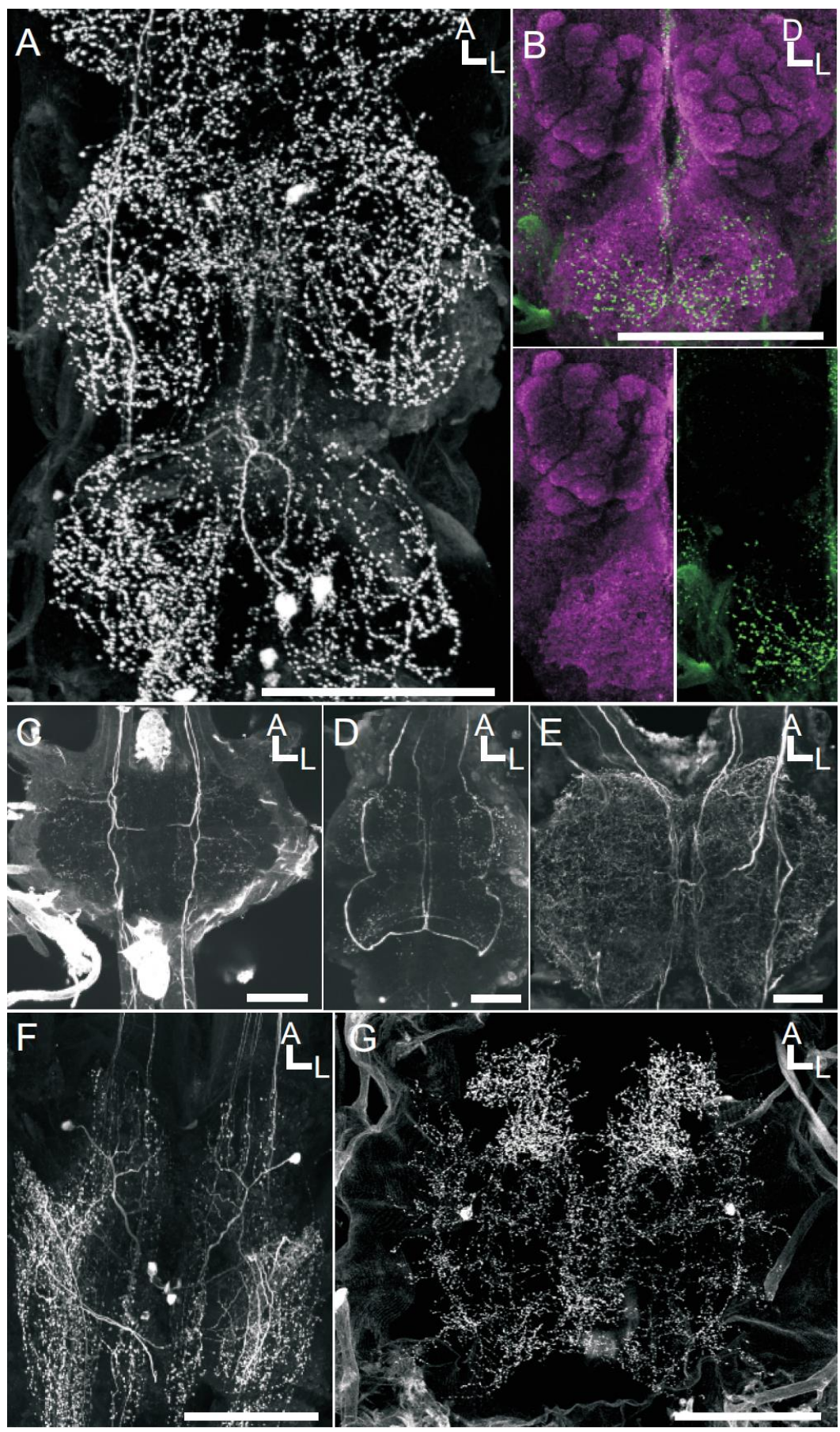


Figure 5:

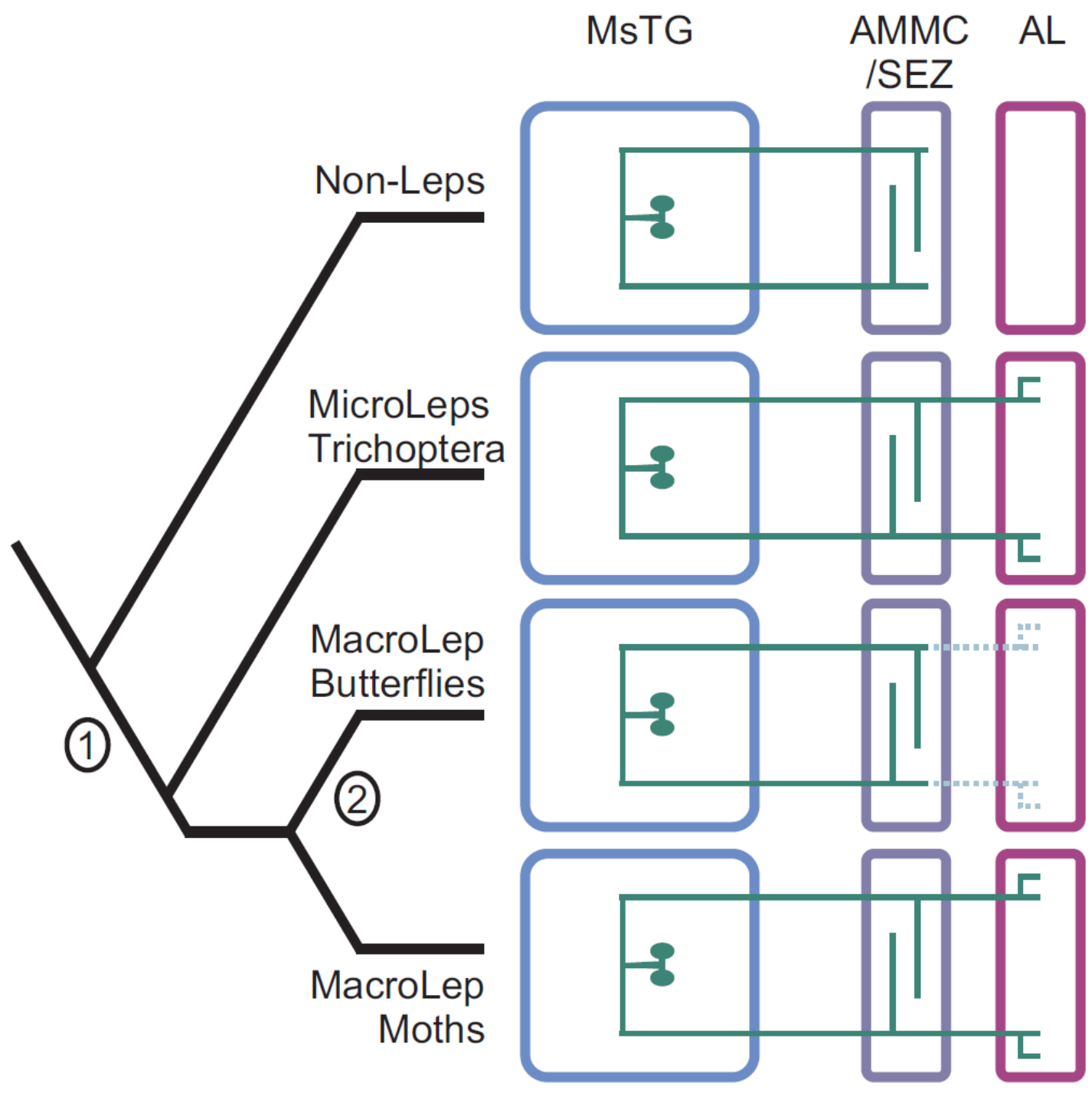

